\title{
OF THE EUROPEAN MATHEMATICAL SOCIETY
}

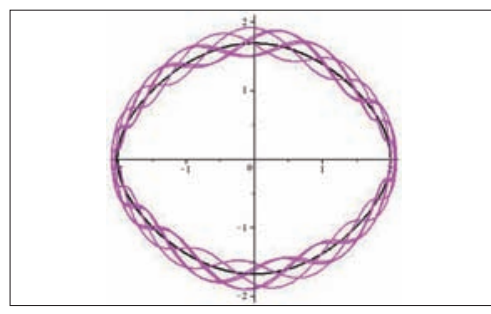

Feature

Three Stories of High Oscillation Mathematics at the Museum

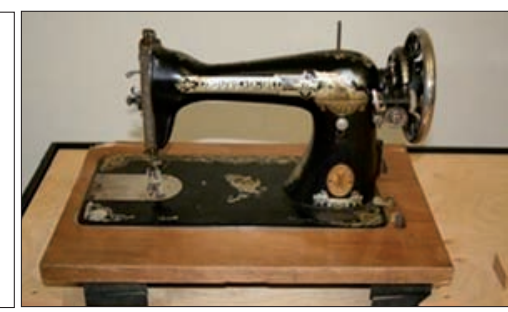

Feature

p. 23

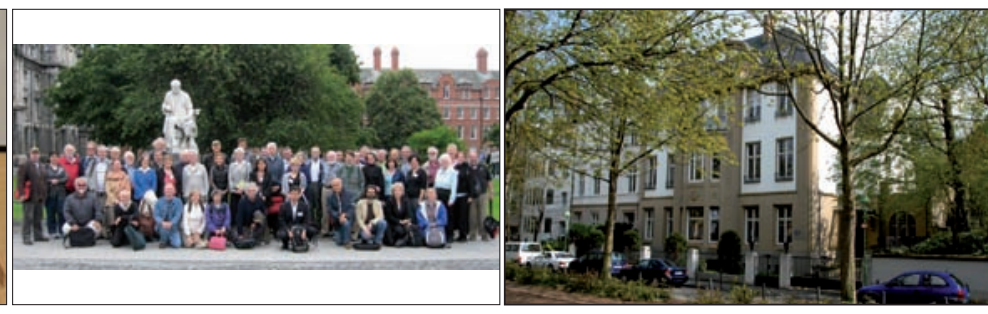

Society

The BSHM

p. 34

Hausdorff Research Institute

p. 18

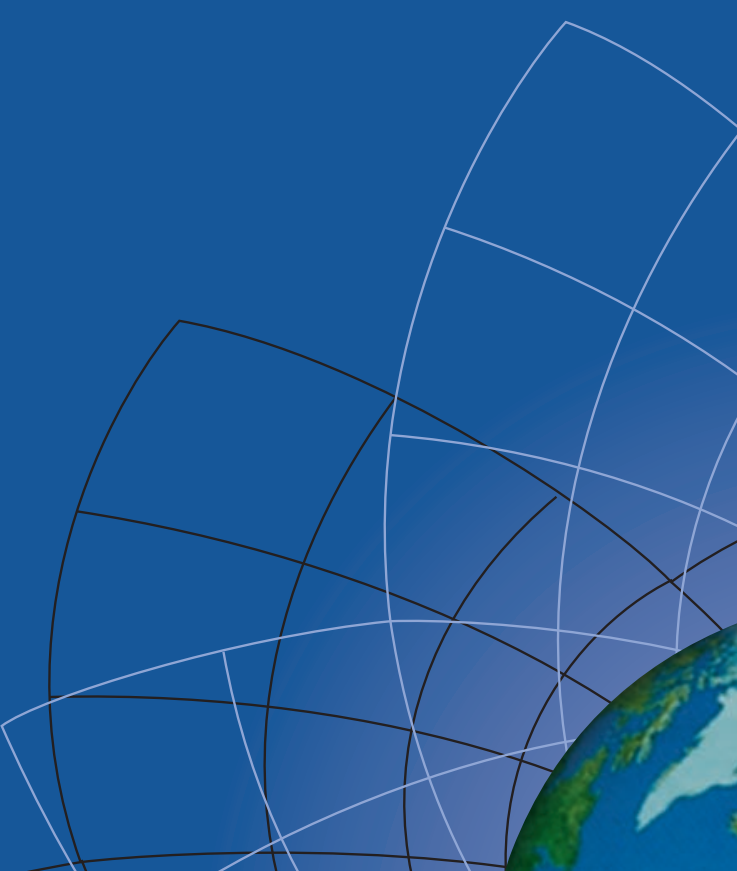

March 2013

Issue 87

ISSN $1027-488 X$

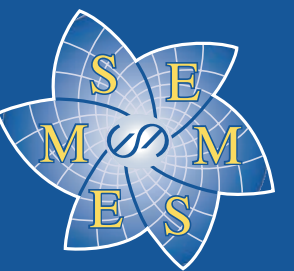

European

Mathematical

Society 


\section{Journals published by the}

\section{Guropean Mathematical Society}

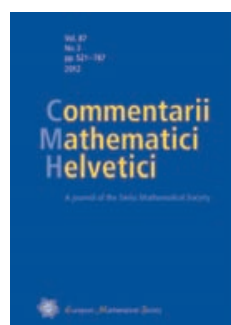

ISSN print $\quad 0010-2571$

ISSN online $1420-8946$

2013. Vol. 88.4 issues

Approx. 1000 pages. $17.0 \times 24.0 \mathrm{~cm}$

Price of subscription:

$328 €$ online only

388 € print+online

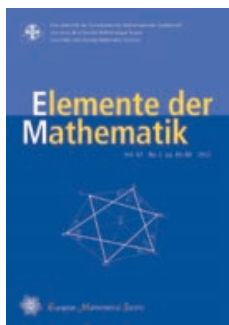

ISSN print 0013-6018

ISSN online 1420-8962

2013. Vol. 68. 4 issues

Approx. 180 pages. $17.0 \times 24.0 \mathrm{~cm}$

Price of subscription:

$74 €$ online only (institutional)

90 € print+online (institutional)

$52 €$ print (individual)

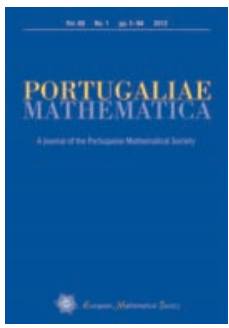

ISSN print $\quad 0032-5155$

ISSN online $1662-2758$

2013. Vol. 69. 4 issues

Approx. 500 pages. $17.0 \times 24.0 \mathrm{~cm}$

Price of subscription:

$196 €$ online only

236 € print+online

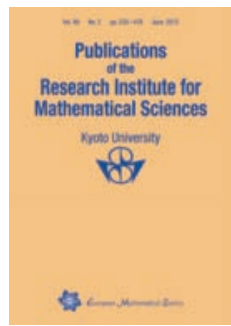

ISSN print $\quad 0034-5318$

ISSN online 1663-4926

2013. Vol. 48.4 issues

Approx. 800 pages. $17.0 \times 24.0 \mathrm{~cm}$

Price of subscription:

$298 €$ online only

348 € print+online

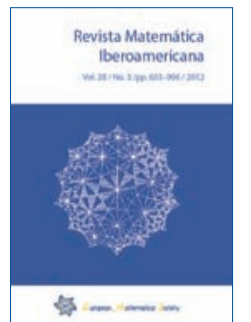

ISSN print $\quad 0213-2230$

ISSN online 2235-0616

2013. Vol. 29. 4 issues

Approx. 1200 pages. $17.0 \times 24.0 \mathrm{~cm}$

Price of subscription:

$328 €$ online only

388 € print+online

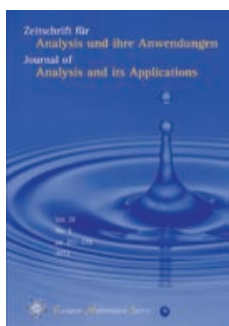

ISSN print $\quad$ 0232-2064

ISSN online 1661-4354

2013. Vol. 32. 4 issues

Approx. 500 pages. $17.0 \times 24.0 \mathrm{~cm}$

Price of subscription:

$190 €$ online only

230 € print+online
A journal of the Swiss Mathematical Society

Editorial Office:

E. Bayer-Fluckiger (École Polytechnique Fédérale de Lausanne, Switzerland)

\section{Aims and Scope}

Commentarii Mathematici Helvetici (CMH) was established on the occasion of a meeting of the Swiss Mathematical Society in May 1928. The first volume was published in 1929. The journal is intended for the publication of original research articles on all aspects in mathematics.

\section{A journal of the Swiss Mathematical Society \\ Managing Editor: \\ Norbert Hungerbühler (ETH Zürich, Switzerland)}

\section{Aims and Scope}

Elemente der Mathematik publishes survey articles about important developments in the field of mathematics; stimulating shorter communications that tackle more specialized questions; and papers that report on the latest advances in mathematics and applications in other disciplines. The journal does not focus on basic research. Rather, its articles seek to convey to a wide circle of readers the relevance, intellectual challenge and vitality of mathematics today. The publication language is primarily German, but many articles are in English, French or Italian.

A journal of the Portuguese Mathematical Society

\section{Editor-in-Chief:}

Luis Nunes Vicente (Universidade de Coimbra, Portugal)

\section{Aims and Scope}

Since its foundation in 1937, Portugaliae Mathematica has aimed at publishing high-level research articles in all branches of mathematics. With great efforts by its founders, the journal was able to publish articles by some of the best mathematicians of the time. In 2001 a New Series of Portugaliae Mathematica was started, reaffirming the purpose of maintaining a high-level research journal in mathematics with a wide range scope.

A journal of the Research Institute for Mathematical Sciences of Kyoto University

Editor-in-Chief:

S. Mochizuki

\section{Aims and Scope}

The aim of the Publications of the Research Institute for Mathematical Sciences is to publish original research papers in the mathematical sciences. Occasionally surveys are included at the request of the editorial board.

A scientific journal of the Real Sociedad Matemática Española

Editors:

Antonio Córdoba (Universidad Autónoma de Madrid, Spain), José L. Fernández (Universidad Autónoma de Madrid, Spain), Luis Vega (Universidad del País Vasco, Bilbao, Spain)

\section{Aims and Scope}

Revista Matemática Iberoamericana publishes original research articles on all areas of mathematics. Its distinguished Editorial Board selects papers according to the highest standards. Founded in 1985, Revista is a scientific journal of Real Sociedad Matemática Española.

\author{
A periodical edited by the University of Leipzig \\ Managing Editors: \\ J. Appell (Universität Würzburg, Germany), M. Günther (Universität Leipzig, Germany), S. Luckhaus \\ (Universität Leipzig, Germany)
}

\section{Aims and Scope}

The Journal for Analysis and its Applications aims at disseminating theoretical knowledge in the field of analysis and, at the same time, cultivating and extending its applications. To this end, it publishes research articles on differential equations, functional analysis and operator theory, notably with applications to mechanics, physics, engineering and other disciplines of the exact sciences. 


\section{Editorial Team}

Editor-in-Chief

Lucia Di Vizio (2012-2016)

Université de Versailles-

St Quentin

Laboratoire de Mathématiques

45 avenue des États-Unis

78035 Versailles cedex, France

e-mail: divizio@math.cnrs.fr

Copy Editor

Chris Nunn

119 St Michaels Road, Aldershot, GU12 4JW, UK e-mail: nunn2quick@qmail.com

Editors

\section{Mariolina Bartolini Bussi}

(Math. Education)

Dip. Matematica - Universitá

Via G. Campi 213/b

I-41100 Modena, Italy

e-mail: bartolini@unimo.it

\section{Jorge Buescu}

(Societies)

Dep. Matemática, Faculdade

de Ciências, Edifício C6,

Piso 2 Campo Grande

1749-006 Lisboa, Portugal

e-mail: jbuescu@ptmat.fc.ul.pt

\section{Eva-Maria Feichtner}

(Research Centres)

Department of Mathematics

University of Bremen

28359 Bremen, Germany

e-mail: emf@math.uni-bremen.de

\section{Eva Miranda}

Departament de Matemàtica Aplicada I, EPSEB, Edifici P

Universitat Politècnica

de Catalunya

Av. del Dr Marañon 44-50

08028 Barcelona, Spain

e-mail: eva.miranda@upc.edu

\section{Mădălina Păcurar}

(Personal Column)

Department of Statistics,

Forecast and Mathematics

Babeș-Bolyai University

T. Mihaili St. 58-60

400591 Cluj-Napoca, Romania

e-mail: madalina.pacurar@econ.ubbcluj.ro;

\section{Ulf Persson}

Matematiska Vetenskaper Chalmers tekniska högskola S-412 96 Göteborg, Sweden e-mail: ulfp@math.chalmers.se

\section{Zdzisław Pogoda}

(Book Reviews) Institute of Mathematicsr Jagiellonian University ul. prof. Stanisława Łojasiewicza 30-348 Kraków, Poland e-mail: zdzislaw.pogoda@uj.edu.pl

\section{Themistocles M. Rassias} (Problem Corner)

Department of Mathematics National Technical University of Athens, Zografou Campus GR-15780 Athens, Greece

e-mail: trassias@math.ntua.gr

\section{Dierk Schleicher}

School of Engineering and

Science

P.O. Box 750561

University Bremen

28725 Bremen, Germany

dierk@jacobs-university.de

\section{Erhard Scholz}

(History of Mathematics)

University Wuppertal

Department C, Mathematics, and Interdisciplinary Center for Science and Technology

Studies (IZWT),

42907 Wuppertal, Germany

e-mail: scholz@math.uni-wuppertal.de

\section{Olaf Teschke}

(Zentralblatt Column)

FIZ Karlsruhe

Franklinstraße 11

10587 Berlin, Germany

e-mail: teschke@zentralblatt-math.org

\section{Jaap Top}

University of Groningen

Department of Mathematics

P.O. Box 407

9700 AK Groningen,

The Netherlands

e-mail: j.top@rug.nl

\section{European \\ Mathematical Society}

Newsletter No. 87, March 2013

EMS Agenda 2

Editorial - L. Di Vizio... 3

New Editors of the EMS Newsletter

4

Message from the President - M. Sanz-Solé ……....................... $\quad \mathbf{5}$

New Members of the Executive Committee .................................. 7

Report on the Council meeting in Helsinki - S. Huggett _ _ _ _..... 9

EMS Home Page with New Look-M. Raussen .................................. 10

About the Code of Practice of the EMS - A. Jensen .......................... 11

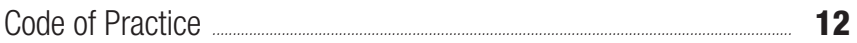

Three Stories of High Oscillation - A. Iserles ................................. 18

Touching the Abstract: Mathematics at the Museum - E. Giusti..... 23

Unity and Disunity in Mathematics - B. Booß-Bavnbek \& P.J. Davis. 28

Episciences: A Publishing Platform for Open Archive

Overlay Journals - J.-P. Demailly.

The British Society for the History of Mathematics -

R. Wilson \& R. Flood 34

Hausdorff Research Institute for Mathematics - W. Lück 37

ICMI Column - M. Bartolini Bussi. 39

Solid Findings - Education Committee of the EMS 42

Current Issues on Mathematics Education Around Europe -

Education Committee of the EMS. 45

Zentralblatt Column - S. Bönisch, G. Greuel \& W. Sperber ... 48

Book Reviews 50

Problem Corner - Th. M. Rassias. 56

The views expressed in this Newsletter are those of the authors and do not necessarily represent those of the EMS or the Editorial Team.

ISSN 1027-488X

(C) 2013 European Mathematical Society

Published by the

EMS Publishing House

ETH-Zentrum SEW A27

$\mathrm{CH}-8092$ Zürich, Switzerland.

homepage: www.ems-ph.org

For advertisements and reprint permission requests contact: newsletter@ems-ph.org 


\section{EMS Executive Committee}

\author{
President \\ Prof. Marta Sanz-Solé \\ (2011-2014) \\ University of Barcelona \\ Faculty of Mathematics \\ Gran Via de les Corts \\ Catalanes 585 \\ E-08007 Barcelona, Spain \\ e-mail:ems-president@ub.edu
}

\section{Vice-Presidents}

\section{Prof. Franco Brezzi}

(2013-2016)

Istituto di Matematica Applicata e Tecnologie Informatiche del

C.N.R

via Ferrata 3

I-27100 Pavia, Italy

e-mail: brezzi@imati.cnr.it

\section{Dr. Martin Raussen}

\section{(2011-2016)}

Department of Mathematical

Sciences, Aalborg University

Fredrik Bajers Vej $7 G$

DK-9220 Aalborg Øst

Denmark

e-mail: raussen@math.aau.dk

Secretary

\section{Dr. Stephen Huggett}

(2007-2014)

School of Computing and

Mathematics

University of Plymouth

Plymouth PL4 8AA, UK

e-mail: s.huggett@plymouth.ac.uk

\section{Treasurer}

\section{Prof. Jouko Väänänen}

(2007-2014)

Department of Mathematics and Statistics

Gustaf Hällströmin katu 2b

FIN-00014 University of Helsinki

Finland

e-mail: jouko.vaananen@helsinki.fi

and

Institute for Logic, Language

and Computation

University of Amsterdam

Plantage Muidergracht 24

NL-1018 TV Amsterdam

The Netherlands

e-mail: vaananen@science.uva.nl
Ordinary Members

Prof. Alice Fialowski

(2013-2016)

Eötvös Loránd University

Institute of Mathematics

Pázmány Péter sétány 1/C

$\mathrm{H}-1117$ Budapest, Hungary

e-mail: fialowsk@cs.elte.hu

(2013-2016)

Mathematical Research

Institute Oberwolfach

Schwarzwaldstr. 9-11

(Lorenzenhof)

D-77709 Oberwolfach-Walke

Germany

e-mail: greuel@mfo.de

\section{Prof. Laurence Halpern}

(2013-2016)

Laboratoire Analyse, Géométrie

\& Applications

UMR 7539 CNRS

Université Paris 13

F-93430 Villetaneuse, France

e-mail: halpern@math.univ-paris13.fr

\section{Prof. Volker Mehrmann}

(2011-2014)

Institut für Mathematik

TU Berlin MA 4-5

Strasse des 17. Juni 136

D-10623 Berlin, Germany

e-mail: mehrmann@math.TU-Berlin.DE

\section{Prof. Armen Sergeev}

(2013-2016)

Steklov Mathematical Institute

Russian Academy of Sciences

Gubkina str. 8

119991 Moscow, Russia

e-mail: sergeev@mi.ras.ru

\section{EMS Secretariat}

\section{Ms. Terhi Hautala}

Department of Mathematics and Statistics

P.O. Box 68

(Gustaf Hällströmin katu 2b)

FIN-00014 University of Helsinki Finland

Tel: (+358)-9-19151503

Fax: (+358)-9-19151400

e-mail: ems-office@helsinki.fi

Web site: http://www.euro-math-soc.eu

\section{EMS Publicity Officer}

\section{Dmitry Feichtner-Kozlov}

FB3 Mathematik

University of Bremen

Postfach 330440

D-28334 Bremen, Germany

e-mail: dfk@math.uni-bremen.de

\section{Prof. Gert-Martin Greuel}

\section{EMS Agenda}

\section{3}

\section{5-7 April}

Joint EMS-DMF Mathematical Weekend, Aarhus, Denmark projects.au.dk/emsweekend/

\section{April}

Meeting of Presidents, Aarhus, Denmark

Stephen Huggett: s.huggett@plymouth.ac.uk

http://projects.au.dk/presidents-ems/

\section{9-20 April}

Committee for Developing Countries meeting, Linköping,

Sweden

Tsou Sheung Tsun: tsou@maths.ox.ac.uk

\section{5-26 May}

Raising Public Awareness Committee meeting, Tycho

Brahe's Island Hven, Sweden

Ehrhard Behrends: behrends@mi.fu-berlin.de

\section{7-31 May}

MS-ESMTB Summer School "Multiscale models in the life sciences", Lyon, France

http://mathbio2013.sciencesconf.org/resource/page/id/5

\section{0-13 June}

26th Nordic-EMS Conference of Mathematicians, Lund, Sweden

Lectures by the EMS lecturer for 2013 Tamar Ziegler

(Technion, Israel)

http://www2.maths.lth.se/nordic26/

\section{8-12 July}

EMS-IML Joint Summer School "The Bellman function technique in harmonic analysis"

http://www.mittag-leffler.se/summer2013/summerschools/ bellman function/

\section{8-13 July}

CIME-EMS Summer School in Applied Mathematics

"Vector-valued Partial Differential Equations and Applications", Cetraro, Italy

http://php.math.unifi.it/users/cime/

20-25 July

29th European Meeting of Statisticians, Budapest, Hungary www.ems2013.eu

\section{2-6 September}

16th Conference of Women in Mathematics, Bonn, Germany Lectures by the EMS lecturer for 2013 Tamar Ziegler

(Technion, Israel)

http://europeanwomeninmaths.org/activities/conference/16th general-meeting-ewm

\section{3-19 October}

IMPAN-EMS Bedlewo School: "EMS School on Computational Aspects of Gene Regulation", Bedlewo, Poland

http://bioputer.mimuw.edu.pl/school.php

\section{2-13 October}

EMS-PTM Joint Meeting "A. Mostowski Centenary", Warsaw, Poland

\section{3-24 November}

Raising Public Awareness Committee meeting, Newcastle, UK Ehrhard Behrends: behrends@mi.fu-berlin.de 


\section{Editorial}

Lucia Di Vizio (Université de Versailles-St Quentin, France)

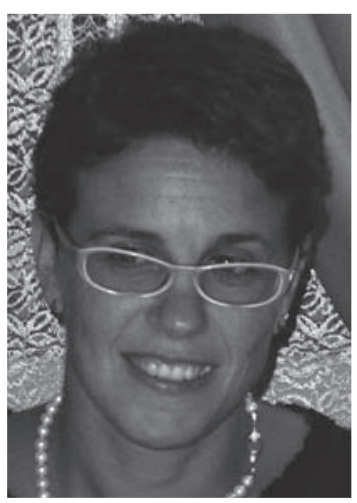

Dear Newsletter readers,

Here I am, writing the editorial of this first issue as sole Editorin-Chief, after six month of joint work with Vicente Muñoz. This adventure started in December 2011, when Mireille MartinDeschamps, former Vice-President of the EMS, first asked me if I was interested in the job. I finally accepted, after a long period of self-questioning. There are many reasons why I was tempted to accept.

First of all, I think that learned societies are important; they should be supported and they can exist only if a majority of their members feel involved and active. I believe in the associative action that is, in our European professional framework, represented by both our national learned societies and the EMS. This is something I have learned to appreciate in France, where associations of citizens play a very important role in society, a complementary role to the one played by institutions. It is also the reason why I have been collaborating with the French Mathematical Society since 2003. Its president at the time was Michel Waldschmidt, whom I am meeting again at the EMS, as the new Chair of the Committee for Developing Countries.

Secondly, I consider myself a European citizen. The mathematical community is very international (I would say cosmopolitan). Nonetheless, our international exchanges are mainly scientific, while many aspects of academic life that we consider and discuss on a national level are actually a European matter. I think this is due to a lack of "familiarity" with our neighbours' daily lives. I would like the Newsletter to be an open forum for discussions, to meet and to know each other better. For this reason we will give more space to readers and members of the EMS in the section "Discussions" and of course the section "Letters to the editors". I therefore invite members of the EMS (both individual and institutional) and readers to submit articles on topics they judge to be of common interest. Along the same line of thought, the editorial board has decided to create a new section called "Across the board: Meeting Point for Europeans Societies". It will contain papers republished from "Newsletters" of other national and European societies, with the purpose of knowing Europe better. In order to enhance this section we are collecting information on their editorial boards, with the help of the recently established net of corresponding members of the EMS. It is my intention to contact the editors-in-chief of the European "Newsletters" in the coming weeks. I take the opportunity of this editorial to ask the editorial boards of those journals which may have been unintentionally forgotten to contact me directly.

A journal like the Newsletter requires a team working together. I have been on the editorial board for only six months and I have to appreciate its atmosphere and the collaboration with colleagues. Therefore, I already have the pleasure of thanking a long list of people who have helped me during my first steps. First of all, I would like to thank Vicente Muñoz for being an attentive guide. He leaves the Newsletter in very good shape, both in terms of the content that is visible to the readers and the production behind the scenes. Indeed, he has put a lot of energy, together with the staff of the EMS Publishing House, into the implementation of the present organisation, with our new submission webpage and, subsequently, a more efficient administration of submitted papers, which benefits future editors. I would also like to thank all the members of the Editorial Board for their warm welcome and the energy and competence they all put into the Newsletter. A special note of thanks must go to the editors who completed their terms of office in December 2012: Vasile Berinde, Chris Budd, Krzysztof Ciesielski, Vicente Muñoz, Frédéric Paugam, Martin Raussen and Robin Wilson. I also take the opportunity to welcome three new editors that have recently joined us: Zdzisław Pogoda, Dierk Schleicher and Jaap Top. Last October, I attended the Helsinki meeting of the Executive Committee. I encourage the readers to follow the periodic reports on its work, which are published in the Newsletter, to fully appreciate the effort and the huge amount of work and energy that our colleagues invest in the EMS, as well as the positive results of these efforts. I would like to thank them all for their support and advice, in particular the President of the EMS Marta Sanz-Solé and the Vice-President Martin Raussen, former Editorin-Chief of the Newsletter. Finally, I would like to thank the staff of the EMS Publishing House, who have always had a very positive reaction to any question or problem, particularly Thomas Hintermann, our copy-editor Chris Nunn, the LaTeX-editor Christoph Eyrich and the whole production team.

The Newsletter should not stop evolving and must always stay attuned to our community and, more generally, to European society. I prefer not to make any programmatic statement: the readers will judge the changes that we are actually able to put into effect. Anyway, I can say that many aspects of the Newsletter need to be considered for improvement: our cover, which is very nice with its focus on Europe in the world, could be revisited; the webpage of the (free) electronic version of the Newsletter could be more friendly for our e-readers; and the list of sections could be reconsidered. The novelty in this 87 th issue is the new agreement with Zentralblatt MATH to republish some of their book reviews. In particular, we 
will give space to Zentralblatt "Looking back reviews", important books that have been milestones in the history of mathematics.

Finally, the Newsletter, and more generally the EMS, needs the support of European mathematicians. Citing the webpage of the EMS, I will say that the principal reasons for joining the EMS are altruistic. Nonetheless, there are benefits for individual members that I will let you discover at http://www.euro-math-soc.eu/node/2083. Of course, one of those benefits is to receive the paper version of the Newsletter in your mailbox!

I am looking forward to receiving more articles and letters from all of you. I wish you a nice reading.

\section{New Editors of the EMS Newsletter}

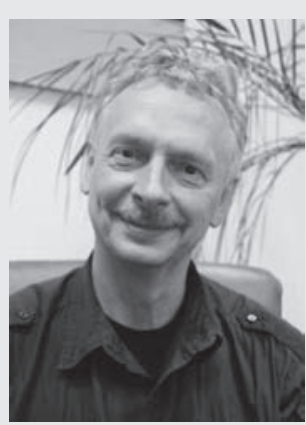

Photo taken by Mrs. Ada Pałka
Zdzisław Pogoda was born in Kraków, 1955. He studied at the Jagiellonian University in Kraków where he received a PhD in 1982. He worked as a lecturer at the Jagiellonian University and he is a professor at the State Higher Vocational School in Nowy Sącz. His specialty is differential geometry and its applications but now he works on the history of mathematics where he is mainly interested in the history of topology (classification problems), geometry (theory of geometric objects) and history of the Krakow Mathematical Center. He is also interested in teacher training, didactics of higher school and popularisation of mathematics. He is an author and co-author (mainly with Krzysztof Ciesielski) of more than a hundred articles and a few books. For his books he has received the prestigious Steinhaus Award from the Polish Foundation for Science Advancement and the Main Dickstein Prize of the Polish Mathematical Society, both with Krzysztof Ciesielski. He likes walking in the mountains, swimming and ride on rollers. He collects minerals and holographic images.

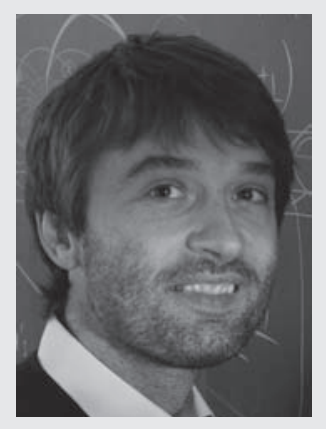

Dierk Schleicher is a professor of mathematics at Jacobs University, Bremen, Germany, where he started in 2001 as the first professor of the then newly founded university. $\mathrm{He}$ studied physics and computer science before obtaining his $\mathrm{PhD}$ in mathematics at Cornell University. In postdoctoral visiting and sabbatical positions, he toured the world, including stays at Berkeley, Stony Brook, Munich, Paris, Toronto and Providence. Much of his research is on dynamical systems, especially holomorphic dynamics. Another focus of his professional activities is on events that bring together young mathematical talents with top mathematical research, such as at the 50th Anniversary of International Mathematical Olympiads (IMO) 2009 or the International Mathematical Summer Schools for Students since 2011. In his free time, he enjoys being outdoors, be it kayaking, sailing, paragliding or hiking in the mountains. 


\section{Message from the President}

Marta Sanz-Solé (University of Barcelona, Spain)

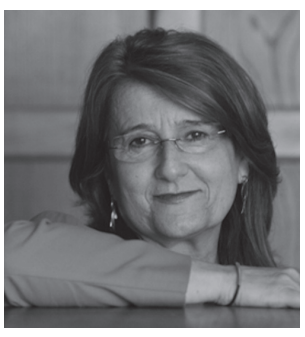

Dear EMS member,

At the beginning of this year, let me express my best wishes for a prosperous and peaceful 2013. It is my pleasure to be in touch with you to report on some aspects of the work of the society in 2012, along with some plans for activities in the near future.

\section{Council and 6ECM in Kraków}

The biennial council and 6ECM, both held in Kraków last July, were among the activities in 2012 that required more concentration. In return, they provided high visibility for the EMS. In the September edition of the newsletter, several articles were devoted to the congress. A detailed report on the council, written by the EMS Secretary Stephen Huggett, appeared in the December edition.

The Polish Mathematical Society and the Jagiellonian University did a tremendous job with the organisation of the congress. National, regional and local governmental institutions provided invaluable support and generous funding. 6ECM was a great and very successful event, an occasion to confirm the good health and vibrant and exciting state of mathematics, and an exceptional showcase for our discipline.

Council attendance was very high. This is a positive sign of the involvement of the membership with the society. One of the council decisions worth noting for your calendars was about the 7th European Congress of Mathematics; it will be held in Berlin on 18-22 July 2016. The first steps for its organisation have already begun.

A new Vice-President Franco Brezzi and four Executive Committee members at large Alice Fialowski, Gert-Martin Greuel, Laurence Halpern and Armen Sergeev were elected by the council. Former Vice-President Mireille Martin-Deschamps together with members Zvi Artstein, Igor Krichever and Rui Loja-Fernandes left the Executive Committee at the end of last year. I take this opportunity to welcome the new members and to express my gratitude to those who stepped down for their devoted work and collaboration with the society.

\section{Publications}

Nowadays, crucial problems related to publication of scientific work are demanding the attention of scientists, publishers and policymakers. The enormous development of information technologies has facilitated the immediate dissemination of new results. This is producing dramatic changes in the classical publication models, with many consequences, in particular on prices. Last summer, the European Commission proposed that Open Access would be the general principle for research funded under Horizon 2020. It also recommended that EU Member States take a similar approach to the results of research funded under their own national programmes. At the council, in a section devoted to publications, some of the implications of these changes were presented at a panel consisting of past presidents Jean-Pierre Bourguignon, Rolf Jeltsch and Ari Laptev. In particular, ethical aspects were considered. The Code of Practice, prepared by the Ethics Committee and approved by the council, is a clear example of EMS commitment to supporting good practices and avoiding unethical behaviour in mathematical publications.

As a learned society, the EMS must build up a position on a sustainable open access publication model that is scientifically reliable and suitable for every mathematician, independent of the workplace. There is also the necessity to design strategies to adapt the EMS Publishing House to this new world, to comply with the principle of good service to the mathematical community that inspired its foundation ten years ago. With these overarching aims, we are now taking the final steps in the formation of an EMS Publications Committee chaired by Bernard Teissier. You will be informed of the composition of the committee very soon.

\section{EU}

With the objective of improving the database of experts in mathematics for evaluation tasks, the EMS has elaborated and delivered to the European Commission a long list of mathematicians from different fields that could collaborate in the assessment of proposals submitted to the EU's calls. This was done after contacts with the Director of European Research Area.

With the winding up of the European Science Foundation, a very useful funding instrument for mathematics, the Research Networking Programmes (RNP), has disappeared. COST Actions are quite similar to RNP in structure although larger in funding. However, COST is only open to projects belonging to some prioritised domains and mathematics is not targeted by any of them. Through direct contacts with the COST President, we are currently devoting efforts to include mathematics as one of the targeted domains.

The Industry, Research and Energy Committee of the European Parliament has been working on the elaboration of the documents for the implementation of Horizon 2020, the EU framework programme for research and innovation for 2014-2020. These documents have to be ap- 
proved by the European Council and by the Parliament. Personal contacts with some members of parliament have provided us with the opportunity to discuss some amendments, to gain more insight on budgetary problems and to have access to politicians and officers for future contacts.

Throughout the year, many activities around Horizon 2020 have been held, like public consultations, workshops and conferences. The EMS has participated in some of them.

Horizon 2020 has not yet been adopted. Its overall budget depends on decisions on the total EU budget. These were to be taken at the Summit of Heads of EU member states held 22-23 November. However, a lack of agreement forced a postponement.

Before the summit, the Initiative for Science in $\mathrm{Eu}$ rope organised a petition for the attention of EU Heads of State against cuts in the EU budget for research, innovation and education, with strong advocacy for the European Research Council (ERC). More than 150,000 scientists and organisations signed. Undoubtedly, ERC grants are of crucial importance for fundamental science. We are already witnessing very beneficial effects on mathematical research. Published statistics on its first five years clearly show the very high success of a group of a few European countries. I had the opportunity to talk with ERC President Helga Nowotny and to agree on the necessity of discussing and eventually implementing measures in order to increase the success of talented researchers working in some geographical areas of Europe.

\section{Activities in 2013}

Throughout this year, the EMS will be involved in many activities. Let me mention a few.

The Danish Mathematical Society (DMF) is celebrating its 140th anniversary. On this occasion, our Danish colleagues have invited the Presidents of EMS member societies to hold their Sixth Meeting in Aarhus on 6 April. In spite of their short history, these meetings have already proved to be excellent opportunities for setting up collaborations within societies, strengthening the links between the EMS and its member societies and nurturing the Executive Committee with ideas for new initiatives. To have more accurate information on the topics that would raise the highest interest from participants, we are currently preparing a questionnaire that will be sent to presidents of national societies. I thank the DMF for the kind invitation and express my congratulations for its achievements and my best wishes for a prosperous future.

In combination with this event, a joint EMS-DMF Mathematical Weekend will take place on 5-7 April, also in Aarhus.

The EMS will be the international partner society of the 26th Nordic Congress of Mathematicians that will take place in Lund on 10-13 June. The congress is dedicated to the memory of the outstanding Swedish mathematician Lars Hörmander, who passed away last November. We feel proud to cooperate in a scientific event with such a longstanding tradition in the Nordic countries.
Two major, year-long, international initiatives will contribute to raising awareness of mathematics not only in scientific environments but also in society at large. These are the Mathematics of Planet Earth 2013 (MPE2013) and the International Year of Statistics (IYS2013). The EMS is a partner of the former and a participant of the latter. The committee Raising Public Awareness of Mathematics is involved in planning and running several activities under MPE2013. As for the contribution to the IYS2013, the EMS and the Bernoulli Society will jointly organise the first of a series of lectures under the theme 'What can statistics/mathematics do for mathematics/statistics?' at the 29th European Meeting of Statisticians, which will take place in Budapest on 20-25 July 2013.

In Europe, the tradition of private sponsorship of projects devoted to mathematics education is neither equally practised in the different geographical areas nor widely acknowledged. By an initiative of the EMS Committee on Education, the meeting 'Friends of Mathematics Education - A European Initiative' will take place in Berlin on 14-15 March. The main objectives are to raise awareness of extant projects funded by different types of organisations, to enhance their impact and interactions and to foster the creation of new initiatives in countries with limited or non-existent traditions.

\section{Closing}

The year 2012 left us with the sad news of the death of the first EMS President Friedrich Hirzebruch. The current successful situation of the EMS owes very much to his wise leadership during the first crucial steps of the society. We honour his memory.

I could not end this report without expressing my gratitude to the Executive Committee members for their continued collaboration. I should also mention the terrific work done within the EMS Committees. Special thanks go to their chairs for leading teams who generously devote part of their time to developing very many EMS projects and to all those who collaborate with us in whatever way.

Marta Sanz-Solé EMS President

Links for items mentioned in the message:

http://www.ems-ph.org/journals/newsletter/pdf/2012-0985.pdf

http://www.ems-ph.org/journals/newsletter/pdf/2012-1286.pdf

http://www.cost.eu/

http://www.no-cuts-on-research.eu/

http://projects.au.dk/presidents-ems/

http://projects.au.dk/emsweekend/.

http://www2.maths.lth.se/nordic26/

http://mpe2013.org/

http://www.statistics2013.org/

http://ems2013.eu/site/index.php?page=en/Home 


\section{New members of the EC of the EMS}

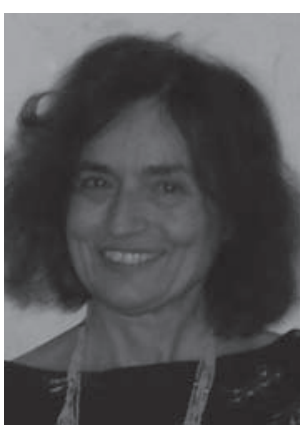

Alice Fialowski was born in Budapest, Hungary. She was educated at Eötvös Loránd University in Budapest. Later she spent three years in Moscow at the Moscow State University where she received the academic degree of candidate of mathematical sciences in 1983. Her thesis adviser was Aleksandr Kirillov. After returning home, she began to receive invitations from Western countries to attend conferences and give talks at universities and institutes. This was the communist era (which ended in 1989). One needed permission to travel. This was not so simple but it gradually became easier. She spent five months in Geneva, three months at MIT and received a Humboldt Fellowship in Germany. She spent two years at the Max Planck Institute for Mathematics in Bonn, where her adviser was Professor Hirzebruch. She was an invited visiting professor at the University of Pennsylvania in Philadelphia. It is there that she met her husband. From Philadelphia she went to the University of California Davis as an associate professor. A few years later she was promoted to full professor. There she also enjoyed working with D. Fuchs and A. Schwarz. In 1994 she returned to her home country where she is on the faculty of Eötvös Loránd University. She and her husband have two daughters.

Her research interests are Lie theory, cohomology, representations, deformation theory and applications in mathematical physics. In California she was one of the founders of the California "Seminars on Lie Groups and Representations". This Seminar met three times a year at different universities. It is a pleasure to report that this seminar is still actively running. By now it has grown into a wider and well-known event. It is similar to the "Sophus Lie Seminar" in Europe, in which she also participates. She has been invited to give lectures in more than 20 countries, including non-European countries such as India, Tunisia and Iran. These have given her insight into quite different cultures. Mathematics is indeed international. She would like to serve the European Mathematical Society to strengthen mathematics both within Europe and internationally.

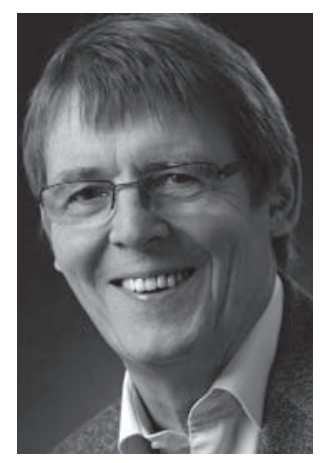

Gert-Martin Greuel studied mathematics and physics at the Universities of Göttingen and Zürich (ETH). He obtained his $\mathrm{PhD}$ in mathematics in 1973 in Göttingen under the direction of Egbert Brieskorn. From 1973 to 1981, he held a position at the University of Bonn. He has spent several research stays abroad, including at the IHES and at the Universities of Nice, Chapel
Hill, Paris VII, UNAM Mexico and Valladolid. From 1981 he has held a full professorship position at the University of Kaiserslautern.

Since 1993 he has been the Director of the Centre for Computer Algebra at the University of Kaiserslautern, since 2002 the Director of the Mathematisches Forschungsinstitut Oberwolfach and since 2010 Chair of European Research Centres of Mathematics (ERCOM). He is Editor-in-Chief of "Zentralblatt MATH" and of "Oberwolfach Reports", Editor of "Ergebnisse der Mathematik und ihrer Grenzgebiete" and Associate Editor of "Revista Matematica Complutense".

His research fields are singularity theory, algebraic geometry and computer algebra. Besides his interests in classification and deformation of singularities he works on algorithms in symbolic computation and their application (together with engineers) to analysis and design of micro electronics systems. He is one of the authors of the computer algebra system Singular. Recently he became active in raising public awareness of mathematics, in particular by directing the exhibition IMAGINARY and by developing it into an interactive open source platform.

In 2009 he received an Honorary Doctorate from Leibniz Universität Hannover and in 2011 he became Honorary Member of the Real Sociedad Matematica Espanola (RSME). He is married and has five children and (so far) 14 grandchildren.

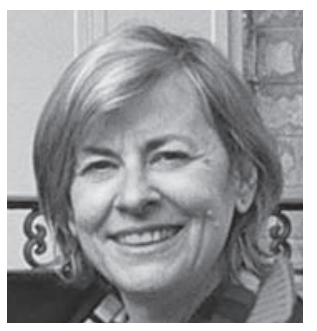

Laurence Halpern received her Doctorat es Sciences from University Paris 6 (France) at Ecole Polytechnique in 1986. She was employed as a CNRS-researcher right after her $\mathrm{PhD}$ in 1981. In 1988, she was named professor at University Paris 13. There, she served for 10 years as the Director of the Engineering School in Applied Mathematics and Scientific Computing and for the last four years has been the Director of the Mathematics Department. She recently joined the Executive Committee of the EMS because she believes in the need to serve the community, to promote strong interactions with industry at the European level and to develop an interest in mathematics among young people in every country.

With respect to areas of research, she is a specialist in mathematical and numerical analysis of problems involving partial differential equations. Her early work pays particular attention to wave model related problems, which have the (justified) reputation of being very delicate to handle. In this area, she has made seminal contributions on the analysis and implementation of:

1. The one-way wave equation.

2. Absorbing boundary conditions. (In this area, she later developed a theory for the case of fluid equations. 
Recently she has turned her attention to PML-based methods and proposed a complete analysis, together with new algorithms.)

3. Domain decomposition methods with the new concept of Optimized Schwarz waveform relaxation.

4. Multi-physics coupling methods with a new approach.

Laurence Halpern's methods apply to a variety of practical problems in electromagnetics, acoustics, elastodynamics and fluid dynamics, which occur in various areas of science and engineering, such as geophysics, environmental sciences, aerospace, electrical and mechanical engineering and material sciences. The work of Laurence Halpern is characterised by a careful and thorough study of partial differential equations, their applicability and, last but not least, the implementability of the developed algorithms. Her investigations have led to a substantial flow of publications in the best journals in her specialty (Numer. Math. and Math. of Comp. among them). She has collaborated with numerous renowned scientists from around the world, including B. Engquist, M. Schatzmann, J.Rauch, N. Trefethen and M. Gander. She has also had many doctoral students, working on both theoretical and industrial subjects. Her connections to industrial problems and scientific computing take place on various levels: as a consultant at ONERA (French aerospace company), as a member of the scientific board of IFP (French petroleum agency) and through contractual $\mathrm{PhD}$ theses. At an international level, she serves as a member of the scientific committee of the domain decomposition community (ddm.org) and as an editor of SIAM J. Scient. Comp. She is involved in the development of a Master's and a doctoral programme in applied mathematics in Vietnam.

She also enjoys teaching mathematics at all levels, with a combination of theorems and numerical experiments.

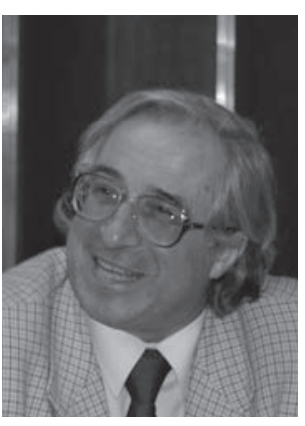

Armen Sergeev graduated from Moscow State University in 1971 and got his $\mathrm{PhD}$ from the same university in 1975 . He went to Steklov Mathematical Institute in 1982 where he occupies the position of deputy director. He got his second doctorate (Doctor of Science) degree from this institute in 1989 . He is also a professor at Moscow State University, Moscow Independent University and the Higher School of Economics.

His research interests concentrate on mathematical physics and several complex variables. His main results include the solution of inhomogeneous Cauchy-Riemann equations in domains generalising product domains (socalled pseudoconvex polyhedra), the solution (together with P. Heinzner) of a compact version of the extended future tube conjecture (the general case was later settled by Zhou Xiangyu), geometric quantisation of infinitedimensional Kaehler manifolds, related to string theory (including loop spaces of compact Lie groups and universal Teichmueller space), investigation of the adiabatic approach to Seiberg-Witten and Ginzburg-Landau equations, and the study of a relation between harmonic spheres in loop spaces and Yang-Mills fields. He has published more than 90 scientific papers in various journals and four monographs.

He is a member of the Ruling Body of Moscow Mathematical Society and the Editor-in-Chief of its journal "Proceedings of Moscow Mathematical Society". He is also Editor-in-Chief of "Steklov Institute Proceedings" and Deputy Editor of "Izvestiya Mathematics". He is one of the organisers of a series of International Conferences on geometric quantisation under the general title GEOQUANT.
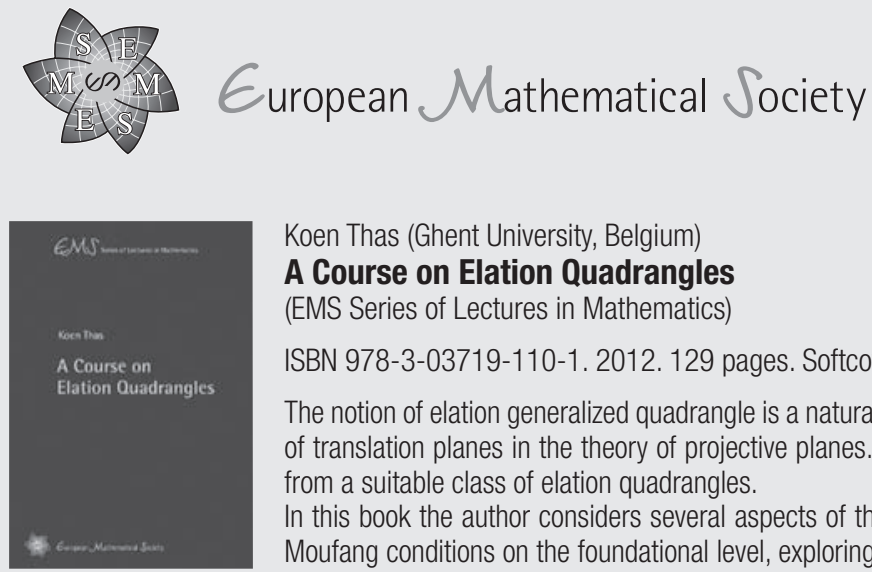

Koen Thas (Ghent University, Belgium)

A Course on Elation Quadrangles

(EMS Series of Lectures in Mathematics)

ISBN 978-3-03719-110-1. 2012. 129 pages. Softcover. 17 x 24 cm. 28.00 Euro

The notion of elation generalized quadrangle is a natural generalization to the theory of generalized quadrangles of the important notion of translation planes in the theory of projective planes. Almost any known class of finite generalized quadrangles can be constructed from a suitable class of elation quadrangles.

In this book the author considers several aspects of the theory of elation generalized quadrangles. Special attention is given to local Moufang conditions on the foundational level, exploring for instance a question of Knarr from the 1990s concerning the very notion of elation quadrangles. All the known results on Kantor's prime power conjecture for finite elation quadrangles are gathered, some of them published here for the first time. The structural theory of elation quadrangles and their groups is heavily emphasized. Other related topics, such as $p$-modular cohomology, Heisenberg groups and existence problems for certain translation nets, are briefly touched.

The text starts from scratch and is essentially self-contained. Many alternative proofs are given for known theorems. Containing dozens of exercises at various levels, from very easy to rather difficult, this course will stimulate undergraduate and graduate students to enter the fascinating and rich world of elation quadrangles. The more accomplished mathematician will especially find the final chapters challenging. 


\section{EMS Executive Committee Meeting in Helsinki on the 27th, 28th, and 29th of October 2012}

Stephen Huggett (University of Plymouth, UK)

The President particularly welcomed the newly elected members of the Executive Committee Alice Fialowski, Gert-Martin Greuel, Laurence Halpern, and Armen Sergeev.

\section{Reports}

The Treasurer gave a detailed explanation of the income and expenditure spreadsheets, noting that the Society's finances are reasonably secure. After some questions, the report was accepted by the Executive Committee.

The President then described the proposal for a project manager, which arose during the Executive Committee's 'brainstorming' meeting last year. The job would be that of a high level secretary, helping the President to prepare position papers and briefings, especially for interactions with the EU. The Executive Committee then agreed to proceed with this suggestion for a trial period of one year.

The Secretary described the work being done on the EMS archive in Helsinki. By the end of 2012 it will have been completely sorted out, and a full index of its contents will have been compiled. The Executive Committee agreed that following this work it may be useful to ask someone to go through the archive as a 'curator', identifying any items of special interest or value.

\section{Scientific meetings}

The Executive Committee started by expressing its heartfelt thanks to the organisers of the 6th European Congress of Mathematics.

Then the President introduced the proposed memorandum of understanding between the Executive Committee and the organisers of the 7th European Congress of Mathematics, emphasising the points that were not in the call for bids. This memorandum was agreed by the Executive Committee. Volker Mehrmann reported on progress in planning for the 7ECM, noting in particular that the web site would be ready by the end of 2012 . The Executive Committee finished by discussing arrangements for the various prizes to be awarded at the 7ECM.

Martin Raussen described the proposal for an EMSDMF Joint Mathematical Weekend in Aarhus from the 5th to the 7th of April 2013. The Executive Committee welcomed the proposal and agreed to appoint Martin as its representative on the Organising Committee.

The President reported on preparations for the EMSNordic Mathematical Congress, noting that Nils Dencker had resigned as an organiser when he became Chair of the EMS Meetings Committee.
The Executive Committee discussed at the joint meeting between the Portugese Mathematical Society and the AMS, in 2015. It was agreed to support this meeting and to engage closely with its Scientific Committee.

\section{Society Meetings}

Martin Raussen reported that the date for the 2013 Meeting of Presidents had been fixed at the 6th of April, and local arrangements were in hand.

Opening the discussion of the meeting of Council in 2014, the President recorded that the proposal to hold it in San Sebastian had been completely independent of her. The Executive Committee accepted the proposal with gratitude, noting that the attached conference to be held in Bilbao could attract 800 participants. The Council will be on the 28th and 29th of June, and the conference from June the 30 th to July the 4 th.

\section{Code of Practice}

The President gave a detailed account of the work on the Code of Practice, noting that the Ethics Committee had modified its earlier draft in response to (a) legal advice, and then (b) comments from the Executive Committee.

The Executive Committee had a long discussion. There was clear agreement from the beginning that the Code itself was excellent and should be adopted immediately. Concerns focussed on the procedural part of the document. Possible outcomes arising from actions taken by the EMS were described, including the outright sacking of the alleged plagiarist, or the whipping up of a political storm. But the danger from libel action against the Society was the most serious, and it was noted that it would not be possible to obtain liability insurance in this case, because to be a crime libel must be deliberate. However, it was stressed that the procedures give very wide discretion to the Society, and no action would be taken by the President without consulting the Executive Committee, and without taking specific legal advice (specific, because it would depend on the jurisdiction in which the alleged offence took place).

The members of the Executive Committee present at the meeting voted, and were unanimous in approving the Code of Practice in full.

\section{Meetings Committee}

The President reported that Nils Dencker had been enthusiastic in his acceptance of the Chair of Meetings Committee. She described the draft paper on calls for EMS scientific activities and sponsorship, which was welcomed by the Executive Committee. 
In discussion, the following points were made.

- The membership of the Meetings Committee will need to be very broad, mathematically.

- The aim should be that the Meetings Committee eventually has its own budget.

- Events should have a clear European dimension, and the Meetings Committee should be active in suggesting them, rather than just responding to suggestions.

- There are some events with a very obvious European dimension which have not so far been related to the EMS in any way. These should be encouraged to seek our sponsorship, at least.

\section{Other Committees}

The Executive Committee agreed a list of names for membership of the future Publications Committee, and also agreed that the remit should in particular make explicit reference to the spectrum of views on open access.

The President presented the proposal from Carles Casacuberta on the evolution of the Committee for Support of East European Mathematicians, which was approved by the Executive Committee.

\section{Publishing}

The Executive Committee discussed the report from Ulf Rehmann on the Encyclopedia of Mathematics wiki, which is highly successful.

The Executive Committee recorded a vote of thanks to Vicente Muñoz for all this excellent work as Editorin-Chief of the Newsletter, and welcomed Lucia Di Vizio to the meeting as Vicente's successor. Lucia gave a short presentation on the Newsletter, emphasising that the new submission system was a great improvement but that the LaTeX papers were still a little awkward to process.

The President reported that the publishing and cooperation agreement on Zentralblatt between the EMS, Fachinformationszentrum Karlsruhe, Heidelberger Akademie der Wissenschaften, and Springer was now signed, and one of the originals is in the EMS office in Helsinki. Finally, Gert-Martin Greuel gave a presentation on Zentralblatt.

\section{Funding Organisations and Political Bodies}

The President gave a brief report on the current budget negotiations for Horizon 2020, noting in particular the tension between funding for the ERC and for Marie $\mathrm{Cu}$ rie, and describing the efforts made by various people to advocate support for research in mathematics.

The petition in support of the European Research Council was discussed briefly, and it was also noted that the ERC has received a very large number of applications for starting grants.

\section{Closing}

The next Executive Committee meeting will be held in Queen's University Belfast, on the 19th, 20th, and 21st of April 2013, on the invitation of Martin Mathieu, President of the Irish Mathematical Society.

The Executive Committee expressed its deep gratitude to the Finnish Academy of Science and Letters and the Finnish Mathematical Society for their warm hospitality. The President also expressed her thanks to all retiring members of the Executive Committee for their hard work.

\section{EMS Home Page with New Look}

Martin Raussen, EMS Vice-President (Aalborg University, Denmark)

The EMS homepage (www.euro-math-soc.eu) has recently undergone a "makeover". In its new form, the central part comprises three columns, listing:

- Activities and Events with EMS involvement.

- News and Headlines of interest for the mathematical community.

- Calls for applications and Documents of high importance for European mathematicians.

May I invite readers to take a regular look at the homepage in order to find important current information or even to install an RSS feed! The EMS site contains a lot more information in submenus. Of particular interest are three sections that you can access from the left menu panel:

- News, a regularly updated list with news concerning mathematics and mathematicians - in a very brief form, including links to background documents.

- Jobs, with international advertisements for mathematicians within and outside academia. This is by far the most visited facility of the entire website. It has re- cently been enhanced so that it is possible to search for specific job categories, countries, etc. ("Search jobs" in the left panel).

- Conferences, with lists of conferences of interest to European mathematicians.

You are invited to submit information in all three categories (again: left panel). Relevant information from your hand will be uploaded as soon as possible.

The recent changes on the website are based on suggestions and comments by many colleagues. Special thanks are due to Alfonso Fuente (Shanghai), Albert Ruiz Cirera (Barcelona) and Paul C. Kettler (Oslo). The EMS website would not even exist without the hard work of our system administrator Matti Pauna (Helsinki).

This is only a preliminary result; a lot of work is still to be done. I would be grateful for your comments and suggestions for improvements and even more so for your active participation in an "editorial board" for the EMS website. Please send messages to raussen@math.aau.dk. 


\section{About the Code of Practice of the European Mathematical Society}

Arne Jensen (Aalborg University, Denmark), Chair, Ethics Committee

The Executive Committee of the European Mathematical Society (EMS) created an Ethics Committee in the Spring of 2010. The first task of the Committee was to prepare a Code of Practice. This task was completed during the Spring of 2012. The Code was approved by the Executive Committee at the end of October and went into effect on 1 November 2012.

\section{The Code of Practice}

The Code is available on the EMS website (see below). It establishes a set of standards to be followed by European mathematicians in their research and professional life, and by editors and publishers of mathematics.

The Code covers the publication and dissemination of mathematical research.

The topics covered are:

- Responsibilities of authors.

- Responsibilities of publishers and editors.

- Responsibilities of referees.

- Responsibilities of users of bibliometric data.

The Code describes good practice and ethical behaviour in the publication, dissemination and assessment of mathematical research, and also describes what is considered to be misconduct or unethical behaviour in these areas.

Concerning authors, it is good practice to give proper credit and to give appropriate bibliographical references to the contributions of others. In recent years plagiarism has become more widespread in the mathematical sciences. Plagiarism is certainly unethical.

Concerning publishers, it is good practice to establish and conspicuously present standards for ethical behaviour in publishing, and specify the steps to be taken to investigate and respond to suspicions or accusations of misconduct.

Concerning editors, they should avoid conflicts of interest, for example by handling their own papers, or those of colleagues, students or acquaintances. If the editors become convinced that parts of a work that they have published have been plagiarised from another source then they should ask the authors to submit a substantial retraction; if this is not forthcoming, they should themselves publish a statement giving details of the plagiarism involved.

Concerning referees, they should avoid professional or personal conflicts of interest. Any case of a possible conflict of interest should be discussed with the editor and the referee can continue to act only with the agreement of the editor. Referees should not use privileged information obtained from a manuscript under review.
Concerning bibliometric data, the users should be aware of the provenance of the data and should use them with sufficient care, understanding the reliability or lack thereof of such data. Authors, editors and publishers should not seek to artificially influence the bibliometric data, impact factors and citation counts that are generated.

\section{Procedures}

As part of the Code, procedures have been established for the consideration of individual cases brought to the attention of the Committee.

Cases can be submitted to the Committee by persons involved in claims of unethical behaviour, as described in the Code. The Committee will not consider third party submissions. Before submission the complainant should have sought to address the issues involved and, in the case of published works, should have utilised the procedures established by the publishers for handling unethical behaviour.

Once the Committee has decided to accept a case, it will seek to discover the underlying facts. The Committee will seek to mediate and, if that fails, it may establish a formal finding. This will be communicated to the President of the EMS, who will then decide how to proceed.

\section{Conclusion}

Enforcement of the Code can only be through moral power, by discouraging unethical behaviour.

The above description is of necessity incomplete. If you are interested in learning more about the Code including the Procedures, please consult the document on the EMS webpage.

This Code is seen as a 'first attempt'. Revision of the Code will be considered in a few years.

Comments on the Code can be sent to the Chairman of the Committee, Professor Arne Jensen.

\section{Reference}

The Code of Practice: http://www.euro-math-soc.eu/system/files/COP-approved.pdf.

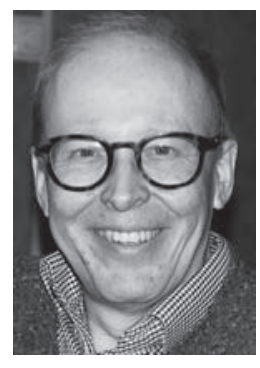

Arne Jensen (matarne@math.aau.dk) got his PhD from the University of Aarhus in 1979. He has been a professor of mathematics at Aalborg University, Denmark, since 1988. He served as acting director of the Mittag-Leffler Institute from 1993 to the beginning of 1995. In 2000/01 he was a visiting professor at the University of Tokyo. His research interests are spectral and scattering theory for Schrödinger operators. 


\section{Code of Practice}

\section{Preamble}

The European Mathematical Society Ethics Committee was created by the Executive Committee of the European Mathematical Society in the spring of 2010. The remit and the list of inaugural members of the Committee are given at the end of this Code.

The first task of the Ethics Committee was to prepare a Code of Practice; this is the present document. The Code was approved by the Executive Committee of the European Mathematical Society on October 29, 2012, on the recommendation of the Council of the European Mathematical Society, and came into effect on November 1, 2012.

The European Mathematical Society recommends that this Code be adhered to by all mathematicians, editors, and publishers of mathematics, especially those based in Europe, but more generally by all who are concerned with the publication, dissemination, and assessment of mathematical research.

It is recommended that this Code of Practice be taken into account by officials of universities and other institutions that employ European mathematicians when transgressions of the Code by their employees are drawn to their attention.

The Code emphasises ethical aspects of publication, dissemination, and assessment of mathematics. The European Mathematical Society considers the successful open and transparent publication and dissemination of mathematical research to be of the greatest importance for the future of our subject. Unethical behaviour in publication and dissemination contaminates and jeopardises the integrity and expansion of mathematics, and could have serious consequences for individuals.

The Code will be revised within three years, in the light of experience with cases analysed, and after consideration of comments received.

The Ethics Committee is willing to consider cases involving allegations of unethical behaviour in the publishing of mathematics. The practices that the Committee intends to follow are laid down in the section 'Procedures', given below.

\section{Code of Practice}

In this section, we set out a code of good practice and ethical behaviour in the publication, dissemination, and assessment of mathematical research, and we specify what we consider to be misconduct or unethical behaviour in this area.

\section{Responsibilities of authors}

1. Individual researchers and authors should understand and uphold high standards of ethical behaviour, particularly in relation to the publication and dissemina- tion of their research. An aspect of good practice is the granting of proper credit, and the referencing of the work of others, with appropriate bibliographic references.

It is important to note that it is not unethical to be mistaken in the attribution, or lack of attribution, of results, provided that authors have carefully sought to determine whether their claimed results are new, and provided that errors of attribution are corrected in a timely and appropriate manner, as they are discovered or pointed out.

Publication of mathematical results as one's own when the author has learned of the results from others, for example through published material, lectures, conversation, or earlier informal publication, constitutes plagiarism: this is a form of theft, is unethical, and constitutes serious misconduct.

2. Each co-author should have contributed significantly to the research reported in any published work, and each person who contributed significantly to the relevant research should be named as a co-author. Further, all named authors should accept joint responsibility for any submitted manuscript and final publication. It is misconduct for one author to submit and to publish joint research without the consent of his or her named co-authors.

3. Most mathematics is published by the submission of manuscripts to journals or conference proceedings (including those that will appear only online), or by the writing of books. Our guiding principle is that an author or authors who submit a work to editors or publishers take responsibility for the integrity of what they have written, seeking carefully to ensure that the mathematics presented is correct and that the work of others is appropriately acknowledged.

4. In mathematics simultaneous or concurrent submission of a manuscript describing the same research to more than one publication constitutes misconduct. Similarly, in mathematics the publication of the same research in more than one journal or outlet without appropriate acknowledgement and citation constitutes misconduct.

5. Translations of published or unpublished works should always fully acknowledge the source of the work.

6. Mathematicians should not make public claims of potential new theorems or the resolution of particular mathematical problems unless they are able to provide full details in a timely manner.

\section{Responsibilities of editors and publishers}

1. It is recommended that journals publishing mathematics should establish and conspicuously present their standards for ethical behaviour in publishing, and specify their responsibilities and the steps to be taken to investigate and respond to suspicions or ac- 
cusations of misconduct. Journals should respond to an author's complaints with respect and due process.

2. Editors should adhere to high standards of ethical treatment of all authors in arriving at a responsible and objective decision about publication. An editor should withdraw from any editorial duties that would involve a personal, commercial, or professional conflict of interest. An editor should also avoid any misuse of their privileged position or of information received as part of their editorial duties to influence the handling of their own papers, or those of colleagues, students, or personal acquaintances. Certainly no information received in confidence should ever be used in the editor's own work.

3. It is recommended that journals publishing mathematics should make clear their policy and practices for handling submissions. In particular, an editor or publisher should acknowledge receipt of a manuscript. A publisher should ensure that the progress of consideration of a submitted manuscript is monitored, and seek diligently to avoid excessive delays in either the refereeing of a paper or the decision process. The publisher must obtain consent to publish either from one author acting on behalf of all authors, or from all authors.

The date of submission of, and the date of any significant changes to, a manuscript should be published; this is important, in particular, in cases of disputes concerning priority.

4. Publishers have an obligation to present mathematical papers and books in a clear and precise format, and they should ensure that the mathematical symbols, words, and sentences that are used in the published work are clear and are not a barrier to understanding. It is misconduct on the part of publishers merely to reproduce without improvement submitted manuscripts that are badly written or presented.

5. Editors and publishers should consider carefully and make objective judgements about the acceptance of submitted manuscripts. Normally this will be on the basis of reports from appropriate referees, but the Committee recognises that it will sometimes be clear to editors that a submitted manuscript is considerably below the standards of the journal, or not in an appropriate subject area, and can therefore be rejected without submission to referees; in this case, the authors should be courteously informed of this rejection in a timely and reasoned manner.

6. The editors should inform potential authors of decisions taken in a courteous and timely manner, always passing on constructive criticism and information provided by the referees. Editors may decide that it is appropriate that certain comments provided by the referees should be confidential to the Editorial Board, and not passed on verbatim to the authors.

7. An author may communicate to the editors the information that a mathematical statement or an attribution in his or her published article is incorrect. In the case where this information is significant, it is recommended that the editors publish a correction or retraction, preferably written by the original author.
8. In some cases, it may be pointed out to the editors by another person that certain statements or attributions in an article appear to be incorrect. In these cases, the editors should consider the comments carefully and react in a proportionate manner; when appropriate, they should insist that the authors write a correction or retraction.

9. In rare cases, the editors may become convinced that parts of a work that they have published have been plagiarised from another source. In these cases, the editors should request the authors to submit for publication a substantial retraction; if this is not forthcoming, the editors themselves should publish a statement giving details of the plagiarism involved.

10. Many articles are first published on the journal web site. It may become apparent that an article so published contains mathematical errors, incorrect attributions, or has been plagiarised in whole or in part. It is recommended that publishers retain the original article for the historical record, but that they indicate by addition at a later specific date appropriate corrections, as they would for a printed article. In extreme cases, it may be that the publishers should indicate that the article has been 'withdrawn' either at the request of the authors or by a decision of the publishers; in this case, any subsequent printed version should reflect this decision.

11. A publisher of journals or books should not list on any of its publications a person as 'editor' or 'editorial advisor' or similar without full disclosure of this to the person concerned and receipt of his or her explicit agreement. The name of any person who resigns from such a position must quickly be removed from the displayed list.

12. Any person listed as editor or editorial advisor should be aware of, and content with, the standards and editorial procedures and policies of the journal, and be willing to act in extreme cases when it is clear that the publishers are not following this Code.

\section{Responsibilities of referees}

1. Referees should adhere to high standards of ethical treatment of all authors in arriving at responsible and objective recommendations about the publication of material that they assess. Referees should seek to validate the correctness, significance, novelty, and clarity of a manuscript under consideration, and then report their findings to the editor in a careful and constructive manner. Nevertheless, final responsibility for the published work lies with the authors.

2. A person asked to accept the task of refereeing a paper may feel that there is a potential personal or professional conflict of interest, for example, when he or she is asked to referee a manuscript from a recent student, collaborator, or colleague. In such cases, the potential referee should discuss with the editor any possible conflicts of interest, and continue to act only with the agreement of the editor.

3. Once they have accepted the task of refereeing a manuscript, referees should seek to report in a timely man- 
ner, taking into account the length of the manuscript and the requests of the editors.

4. A referee should eschew the use of privileged information gleaned from a manuscript under review.

5. A referee who suspects any element of plagiarism in a manuscript under consideration, or any other unethical behaviour, should quickly report these concerns to the editor.

\section{Responsibilities of users of bibliometric data}

1. Whilst accepting that mathematical research is and should be evaluated by appropriate authorities, and especially by those that fund mathematical research, the Committee sees grave danger in the routine use of bibliometric and other related measures to assess the alleged quality of mathematical research and the performance of individuals or small groups of people.

2. It is irresponsible for institutions or committees assessing individuals for possible promotion or the award of a grant or distinction to base their decisions on automatic responses to bibliometric data.

3. It is unethical to manipulate references within an article or to arrange the publication of articles for the purpose of artificially influencing the bibliometric data, impact factors, and citation counts that are generated.

4. It is unethical to include inappropriate citations of one's own work or of the work of particular colleagues or of articles in journals with which the author has a connection.

5. It is misconduct for publishers to advertise their own journals by the quotation of insecure or partial or tendentious bibliometric data.

\section{Procedures}

The following procedures will guide the considerations of individual cases that are brought to the attention of the Ethics Committee.

P1 The Committee will consider only cases that are formally submitted to it by persons or bodies that are involved in claims of unethical behaviour. The Committee will not consider cases submitted by those who have no standing in a dispute, and the Committee will not itself seek out instances of apparent unethical behaviour.

The Committee may decline to act on any case that is brought to its attention. The Committee will not reconsider a case after a decision has been made unless substantial new information which could lead to a different decision is made available.

P2 Cases for consideration should be communicated to the Chairman of the Committee.

Although the Committee will not act until a formal complaint is lodged, earlier informal enquiries may be addressed to the Chairman.

P3 The Committee expects that before submitting a case a complainant will have already sought to address the issues involved and, in the case of published works, will have utilised the procedure for dealing with ethical issues formulated by the publishers.
P4 The Committee will not consider any case in which formal legal proceedings have been instigated, and may cease to consider a case if such proceedings are commenced. The Committee will not consider any case that is a matter of direct dispute between a mathematician and the institution that employs that person.

P5 The normal procedure of the Committee when it receives a formal complaint will be as follows.

First, the Committee will determine whether it is appropriate to consider the complaint and whether a prima facie case exists.

If it does so determine, the Committee will then seek to discover the underlying facts of a case. As part of this process, the Chairman will write privately to the accused person or bodies, and invite them either to act quickly to accept the complaint and make appropriate amends, or to explain to the Committee why they do not deem it appropriate to act in this way.

In the latter case, or when the accused party does not respond, especially when accusations of plagiarism are made, the Committee will normally ask some experts, each unconnected to the various parties, to study the accusations and advise the Committee whether they are justified. On receipt of this advice, the Committee will form a view on the merits of the case, and will then communicate its findings privately to all parties.

The Committee expects that any party deemed to have acted unethically will make appropriate and timely amends.

P6 In the case where the party deemed to have acted unethically remains obdurate, and the Committee is convinced that unethical behaviour has occurred, the Committee will make a formal finding, which will be sent by the Chairman to the President of the European Mathematical Society.

The President, after consultation with the Executive Committee, may communicate the findings, for example by informing the Head of the Institution that employs the party deemed to have acted unethically, the relevant Heads of Department of people involved, relevant editors and publishers, as appropriate.

The European Mathematical Society may publicise the findings of the Ethics Committee in a particular case.

P7 The Committee will report regularly on its activities and summarise its findings, without identifying persons or institutions involved in specific cases, in the Newsletter of the European Mathematical Society.

Members of the Ethics Committee will adhere to the following principles.

- Each member of the Committee will excuse himself or herself from the discussion of and any participation in the decision concerning any case submitted to the Committee if they have any conflict of interest (or anything that could give an appearance of a conflict of interest) related to the submitted case. Such a Committee member should inform the Chairman in advance, and then he or she will not receive any papers or information related to the relevant case. 
- All members of the Committee will keep all cases confidential until a decision has been made public; all internal discussions and information received concerning individuals will remain confidential.

\section{Remit}

The remit of the European Mathematical Society Ethics Committee was specified by the Executive Committee of the European Mathematical Society in Spring 2010, as follows.

The Ethics Committee will focus on unethical behaviour in mathematical publications. This includes, for example, plagiarism, duplicate publication, inadequate citations, inflated self citations, dishonest refereeing, and other violations of the professional code. The Committee will be responsible for the following three tasks:

1. To raise the awareness of the problem by preparing $a$ code of practice.

2. To encourage journals and publishers to respond to allegations of unethical behaviour in a conscientious way.

3. To provide a mechanism whereby researchers can ask the Committee to help them pursue claims of unethical behaviour.

The Committee may take up any other relevant questions related to ethics in connection with its work.

\section{Committee}

The initial membership of the European Mathematical Society Ethics Committee was as follows.

All members were appointed for three years, from mid2010 to mid-2013. Members serve on the Committee as individuals, and not as representatives of their institution, mathematical society, or country.

Chairman: Arne Jensen (Aalborg Universitet, Denmark) Vice-Chairman: H. Garth Dales (University of Lancaster, UK)

Executive Committee representative: 2010-2012: Igor Krichever (Columbia University, New York, USA, and Landau Institute of Theoretical Physics, Moscow, Russia), 2013- Franco Brezzi (Istituto Universitario di Studi Superiori, Pavia, Italy.

Members:

Jean-Paul Allouche (Centre National de la Recherche Scientifique and Université Pierre et Marie Curie, Paris, France)

Graziano Gentili (Università di Firenze, Italy)

Radu Gologan (Academia Română de Științe, București, Romania)

Christine Jacob (Institut National de la Recherche Agronomique, Jouy-en-Josas, France)

Adolfo Quirós (Universidad Autónoma de Madrid, Spain)

Tomaž Pisanski (Univerza v Ljubljani, Slovenia)

Tatiana Shaposhnikova (Linköpings Universitet, Sweden)

\begin{tabular}{|c|c|c|}
\hline & $\begin{array}{l}\text { New books by the } \\
\text { European Mathematical Society }\end{array}$ & $\begin{array}{l}\text { European Mathematical Society Publishing House } \\
\text { Seminar for Applied Mathematics } \\
\text { ETH-Zentrum SEW A27, CH-8092 Zürich, Switzerland } \\
\text { orders@ems-ph.org / www.ems-ph.org }\end{array}$ \\
\hline $\begin{array}{l}\text { Pater Raguetele } \\
\text { Contributions } \\
\text { to the Hilitory of } \\
\text { Number Theory } \\
\text { in the } 20 \text { th Century }\end{array}$ & \multicolumn{2}{|c|}{$\begin{array}{l}\text { Peter Roquette (University of Heidelberg, Germany) } \\
\text { Contributions to the History of Number Theory in the 20th Century } \\
\text { (Heritage of European Mathematics) } \\
\text { ISBN 978-3-03719-113-2. 2013. } 289 \text { pages. Hardcover. } 17 \times 24 \mathrm{~cm} \text {. } 78.00 \text { Euro } \\
\text { The 20th century was a time of great upheaval and great progress, mathematics not excluded. In order to get the overall picture of } \\
\text { trends, developments and results it is illuminating to look at their manifestations locally, in the personal life and work of people living at } \\
\text { the time. The university archives of Göttingen harbor a wealth of papers, letters and manuscripts from several generations of mathema- } \\
\text { ticians - documents which tell us the story of the historic developments from a local point of view. } \\
\text { The present book offers a number of essays based on documents from Göttingen and elsewhere - essays which are not yet contained } \\
\text { in the author's Collected Works. These little pieces, independent from each other, are meant as contributions to the imposing mosaic of } \\
\text { They are written for mathematicians but with no special background requirements. Involved are the names of Abraham Adrian Albert, } \\
\text { hard Brauer, Otto Grün, Helmut Hasse, Klaus Hoechsmann, Robert Langlands, Heinrich-Wolfgang Leopoldt, Emmy Noether, Abraham } \\
\text { Hermann Weyl and others. }\end{array}$} \\
\hline $\begin{array}{l}\text { Patere M Meuman } \\
\text { The mathematical } \\
\text { Writings of } \\
\text { Evariste Galois }\end{array}$ & $\begin{array}{l}\text { Peter M. Neumann (University of Oxford, UK) } \\
\text { The mathematical writings of Évariste Galois } \\
\text { (Heritage of European Mathematics) } \\
\text { ISBN 978-3-03719-104-0. 2011. } 421 \text { pages. Hardcover. } 17 \\
\text { Although Évariste Galois was only } 20 \text { years old when he died, } \\
\text { of algebra. He invented what is now called Galois Theory, the } \\
\text { purpose, and in particular to formulate a precise condition for } s \\
\text { vestigating their theory. His main writings were published in Fr } \\
\text { The present work contains English translations of almost all t } \\
\text { the original French, and are enhanced by three levels of comn } \\
\text { publications in which it appears, and the vagaries of his manu }\end{array}$ & $\begin{array}{l}\text { when they were published } 14 \text { years later, changed the course } \\
\text { worm of what was classically the Theory of Equations. For that } \\
\text { of equations by radicals, he also invented groups and began in- } \\
846 \text {. Very few items have been available in English up to now. } \\
\text { material. They are presented alongside a new transcription of } \\
\text { in introduction explains the context of Galois' work, the various }\end{array}$ \\
\hline
\end{tabular}




\section{The 26th Nordic and 1st European- Nordic Congress of Mathematicians}

\section{Lund, Sweden, 10-13 June 2013}

\section{Sandra Pott (Lund University, Sweden)}

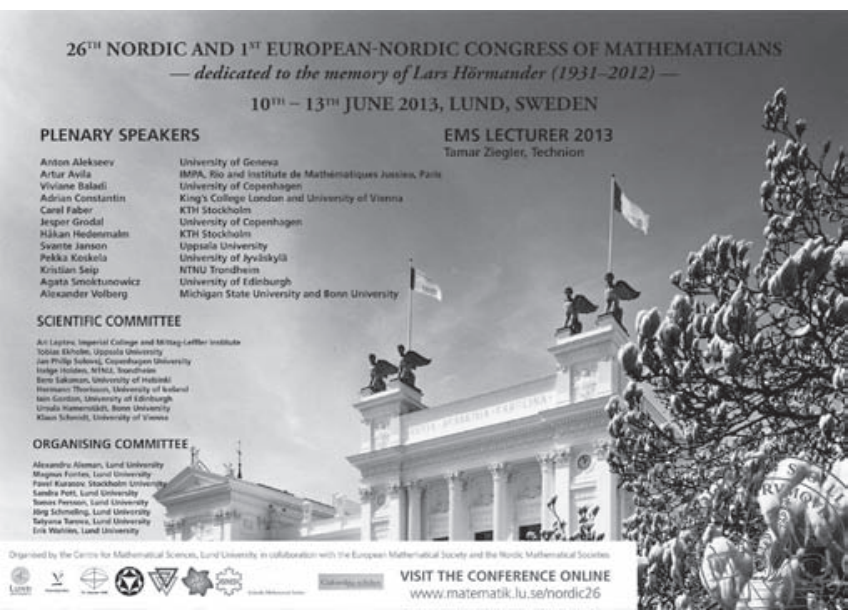

The Nordic Congress of Mathematicians has a long and splendid history, starting with the first Congress in Stockholm in 1909. It takes place every four years and plays a central role in the mathematical life of the Nordic Countries. In 2013, the Congress returns to Sweden, exactly 60 years after the celebrated 12th Congress of Mathematicians in Lund in 1953. It will take place at the Centre for Mathematical Sciences at Lund University and will be the first joint Congress of the European Mathematical Society and the Nordic Mathematical Societies.

The Congress is dedicated to the memory of Lars Hörmander, who was a professor in Lund for many years.

\section{EMS lectures: Tamar Ziegler}

Plenary speakers: Anton Alekseev, Artur Avila, Viviane Baladi, Adrian Constantin, Carel Faber, Jesper Grodal, Håkan Hedenmalm, Svante Janson, Pekka Koskela, Vladimir Maz'ya Kristian Seip, Agata Smoktunowicz, Xavier Tolsa

Special sessions include: Approximation, Control Theory, Dynamical Systems, Harmonic Analysis, Homological Methods, Mathematics Education, Nonlinear PDE, Operator Theory, Probability, Pseudodifferential Operators, and Operator Algebras

Webpage: www.matematik.lu.se/nordic26

\section{CIM International Conferences and Advanced Schools Planet Earth, Portugal 2013}

\section{Alberto Adrego Pinto, CIM President (University of Porto, Portugal)}

The International Center of Mathematics (CIM) is a partner institution of the international programme Mathematics of Planet Earth 2013 (MPE 2013). CIM plans to organise and support several activities within the scope of Mathematics of Planet Earth 2013 (see http://sqig. math.ist.utl.pt/cim/mpe2013/).

To this extent, CIM is organising the following CIM International Conferences and CIM Advanced Schools Planet Earth:

MECC 2013 - International Conference and Advanced School Planet Earth, Mathematics of Energy and Climate Change, 18-28 March 2013.
Keynote speakers and school lecturers: Inês Azevedo, Carnegie Mellon University, USA; Richard James, University of Minnesota, USA; Christopher K. R. T. Jones, University of North Carolina, USA; Pedro Miranda, Universidade de Lisboa, Portugal; Keith Promislow, Michigan State University, USA; Richard L. Smith, University of North Carolina, USA; José Xavier, Universidade de Coimbra, Portugal; David Zilberman, University of California, Berkeley, USA.

DGS 2013 - International Conference and Advanced School Planet Earth, Dynamics, Games and Science, 26 August to 7 September 2013.

Keynote speakers and school lecturers: Michel Benaim, Université de Neuchâtel, Switzerland; Jim Cushing, Uni- 
versity of Arizona, USA; João Lopes Dias, Universidade Técnica de Lisboa, Portugal; Pedro Duarte, Universidade de Lisboa, Portugal; Diogo Gomes, Universidade Técnica de Lisboa, Portugal; Yunping Jiang, City University of New York, USA; Eric Maskin, Institute for Advanced Studies, USA (schedule permitting); Jorge Pacheco, Universidade do Minho, Portugal; David Rand, University of Warwick, UK; Martin Shubik, Yale University, USA (video lecture); Satoru Takahashi, Princeton University,
USA; Marcelo Viana, Instituto de Matemática Pura e Aplicada IMPA, Brazil.

The first two volumes of the CIM Series in Mathematical Sciences published by Springer-Verlag will consist of selected works presented at the conferences Mathematics of Planet Earth (CIM-MPE). The editors of these first two volumes are Jean Pierre Bourguignon, Rolf Jeltsch, Alberto Pinto and Marcelo Viana.

\section{European Women in Mathematics and European Mathematical Society 2014 Summer School at the Institut Mittag-Leffler} Call for Proposals

EWM and the EMS Women in Mathematics Committee are pleased to invite proposals for a one week summer school at the Institut Mittag Leffler in Stockholm in summer 2014.

There are places for about 20 young people and two invited lecturers. The Institut will cover lodging and a meal allowance for everyone. The school should be on a focussed topic of current importance and open to $\mathrm{PhD}$ students and post-docs of both sexes.

A special feature of the summer school is that there will be a much larger than usual involvement by women. We expect that most or all of the organising committee, at least half the participants, and if possible the lecturers, should be female.

There may be some scope for slightly increasing the number of participants or lecturers; this will have to be negotiated with the Institut nearer the time, as will the exact dates. Participants are expected to fund their own travel expenses, and organisers will have to find additional funding for the speakers, as well as any additional participants. The EMS is supportive of this project and it is likely that they will be ready to assist with some of the necessary additional funding. Additional help and advice will also be given by EWM and the EMS committee.

Proposals, which should be framed according to the guidelines which can be found on the EWM website http:// ewm-association.org/ (under 'News'), should be sent electronically as a pdf file to secretary@mittag-leffler.se with a copy to susanna.terracini@unito.it by March 15th 2013. They will be selected by the committee on the basis of their scientific merit and the extent to which they fulfill the aims of the guidelines. In case of any difficulty, please contact either Susanna Terracini or Caroline Series C.M.Series@warwick.ac.uk

\section{Erratum}

In the EMS Newsletter No. 86, Article "The Hirsch Conjecture Has Been Disproved: An Interview With Francisco Santos" by Eva Miranda, page 33, right column, line 36, the correct number of vertices is 36425 and not 36442. 


\section{Three Stories of High Oscillation}

Arieh Iserles (University of Cambridge, UK)

\section{1}

\section{Think globally, act globally!}

Numerical analysis - indeed, mathematical analysis - is in large measure a story of the local. Sufficiently smooth functions are approximated very well in a small neighbourhood by polynomials, indeed even by linear functions. A case in point is the granddaddy of all discretisation methods for differential equations, the Euler method: given the ordinary differential system $\boldsymbol{y}^{\prime}=\boldsymbol{f}(t, \boldsymbol{y})$ and having already found $\boldsymbol{y}_{n} \approx \boldsymbol{y}\left(t_{n}\right)$, we approximate $\boldsymbol{y}\left(t_{n+1}\right)$, where $t_{n+1}=y_{n}+h$, by $\boldsymbol{y}_{n}+h \boldsymbol{f}\left(t_{n}, \boldsymbol{y}_{n}\right)$. In other words, we progress from $t_{n}$ to $t_{n+1}$ assuming that locally the solution is linear, uniquely determined from its value and its slope at $t_{n}$. As long as $\boldsymbol{f}$ is twice smoothly differentiable, the error is $O\left(h^{2}\right)$ and it can be made sufficiently small by choosing small step-size $h>0$. Of course, in place of linear functions, we can use polynomials, say, or rational functions of increasing degree of sophistication and intricacy but the same principle applies: we zoom on a sufficiently small neighbourhood. Reductio ad absurdum of numerical analysis would leave us with a single phrase, "the Taylor theorem".

As long as functions are 'nice', the Taylor theorem is a reasonable foundation stone to numerical algorithms. It is difficult to imagine the modern world without scientific computing and its many achievements: acting locally to think globally is the right strategy most of the time. Yet, important exceptions abound and they come in two flavours. Some functions are not sufficiently smooth to be approximated well by, say, polynomials, calling for more subtle forms of discretisation. Other functions might be misleadingly 'nice', yet their derivatives are large, rendering their local approximation with the Taylor theorem very poor indeed. This is in particular the case with highly oscillating functions because, once Taylor series are truncated, the error scales like a (high) derivative but, each time we differentiate, the amplitude is multiplied by frequency.

An accessible example is provided by the Airy equation $y^{\prime \prime}+t y=0$. The solution behaves for large $t$ as $y(t) \sim$ $c t^{-1 / 4} \sin \left(\frac{2}{3} t^{3 / 2}\right)-$ it oscillates increasingly fast as $t$ grows [13]. It is easy to confirm that $\left.y^{(m)}(t) \sim c t^{m / 2-1 / 4} \sin \left(\frac{2}{3}\right)^{3 / 2}\right), m \geq 0$, and the amplitude of derivatives increases rapidly for $t \gg 1$. This is demonstrated in Fig. 1.1. Thus, as $t$ grows, $y(t)$ is poorly approximated by polynomials and increasing the polynomial degree actually makes things worse!

High oscillation is present in a very wide range of phenomena and numerical modelling of high-frequency phenomena is vital. Yet, the Taylor theorem alone is inadequate for this purpose, calling for an entirely new breed of discretisation methods. One such type of methods endeavours to bring together numerical analysis and asymptotic expansions, marrying local behaviour with global features of the solution. Three such scenarios are described in this paper: the computation of highly oscillatory integrals, the discretisation of ordinary differential equations with highly oscillatory forcing

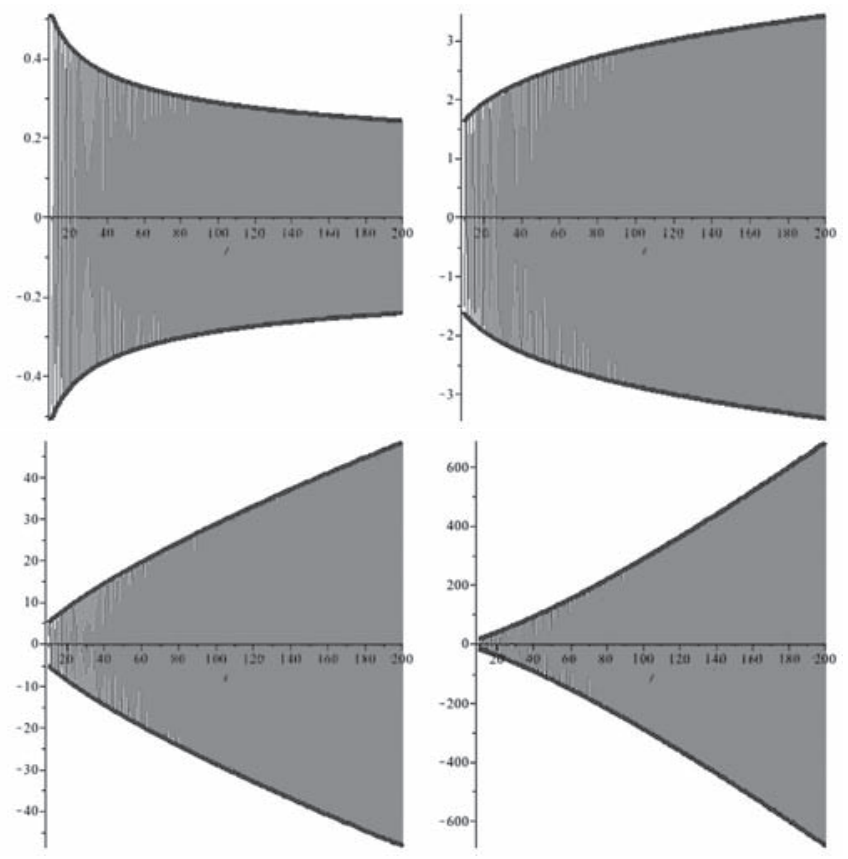

Figure 1.1. The solution of $y^{\prime \prime}+t y=0, y(0)=1, y^{\prime}(0)=0$ and its first, second and third derivatives. The thick black line depicts the envelope $\pm 0.91 t^{m / 2-1 / 4}$.

terms and the computation of the linear Schrödinger equation. In none of the cases have we tried to achieve the greatest possible generality or sophistication, opting instead for accessible examples of numerical methods where global behaviour informs computation.

\section{Story 1: Highly oscillatory quadrature}

Computing integrals is an art as old as integration itself and the oldest quadrature method is due to Sir Isaac Newton (although, in fairness to Archimedes, he used something very much like quadrature to calculate the area of a disc). This is the lore of undergraduate numerical analysis courses: given a weight function $w(x) \geq 0$, not identically zero, and assuming that everything in sight is smooth,

$$
\int_{a_{-}}^{a_{+}} f(x) w(x) \mathrm{d} x \approx Q_{\nu}[f]=\sum_{m=1}^{v} b_{m} f\left(c_{m}\right),
$$

where the nodes $c_{1}, \ldots, c_{v}$ are distinct numbers in $\left[a_{-}, a_{+}\right]$, while $b_{1}, \ldots, b_{v}$ are the quadrature weights. Other things being equal, the best nodes are the zeros of the $v$-degree orthogonal polynomial with respect to the $\mathrm{L}_{2}$ inner product generated by the weight function $w$ and the outcome, Gaussian quadrature, is exact for all polynomials $f$ of degree $\leq 2 v-1$.

Let

$$
I[f](\omega)=\int_{-1}^{1} f(x) \mathrm{e}^{\mathrm{i} \omega x} \mathrm{~d} x .
$$

Once $\omega$ becomes large, the integrand in (2.1) oscillates at an 
increasingly faster pace. In Fig. 2.2 we display the magnitude of the error once Gaussian quadrature is applied to $I[f](\omega)$ for $0 \leq \omega \leq 50$. The pattern is plain to see. For small $\omega$ Gaussian quadrature performs very well and delivers remarkable accuracy: the larger $v$, the better. However, once $\omega$ grows, roughly when $\omega v>1$, accuracy drops sharply and, after a short while, (2.1) fails to deliver even a single significant digit!

The reason is plain to see: the integrand in (2.1) is very poorly approximated by polynomials of reasonable degree. We are precisely in the regime where the Taylor theorem is of little use! Thus, we turn to asymptotics for help. It is easy to prove that

$I[f](\omega) \sim-\sum_{n=0}^{\infty} \frac{1}{(-\mathrm{i} \omega)^{n+1}}\left[f^{(n)}(1) \mathrm{e}^{\mathrm{i} \omega}-f^{(n)}(-1) \mathrm{e}^{-\mathrm{i} \omega}\right], \quad \omega \gg 1$, and this indicates the asymptotic method

$$
A_{s}[f](\omega)=-\sum_{n=0}^{s} \frac{1}{(-\mathrm{i} \omega)^{n+1}}\left[f^{(n)}(1) \mathrm{e}^{\mathrm{i} \omega}-f^{(n)}(-1) \mathrm{e}^{-\mathrm{i} \omega}\right]
$$

[9]. Note that the error, $O\left(\omega^{-s-2}\right)$, improves for increasing $\omega$ !

Fig. 2.3 on the left displays the error (in a format identical to Fig. 2.2) incurred by (2.2) for $s=0,1,2,3$, except that we have taken a much larger range of $\omega$. The comparison with Gaussian quadrature is striking: for large $\omega$ we require much less information, yet obtain a surprisingly precise answer. The quid pro quo is that, having committed all our assets to recover 'large $\omega$ ' behaviour, we can hardly complain that the asymptotic method is useless for small $\omega>0$. In a sense, Gaussian quadrature and the asymptotic method are complementary.

Which brings us to the bottom of Fig. 2.3. We see there for large $\omega$ behaviour somewhat better than (2.2) but careful examination of the neighbourhood of the origin indicates that the mystery method performs well also for small $\omega$. Since the extra expense of the mystery method, compared with (2.2), is marginal, we indeed enjoy the best of all worlds: an asymptotic-numerical method.

So, what is the mystery method? Given the nodes $c_{1}=$ $-1<c_{2}<\cdots<c_{v-1}<c_{v}=1$, each $c_{k}$ with a multi-

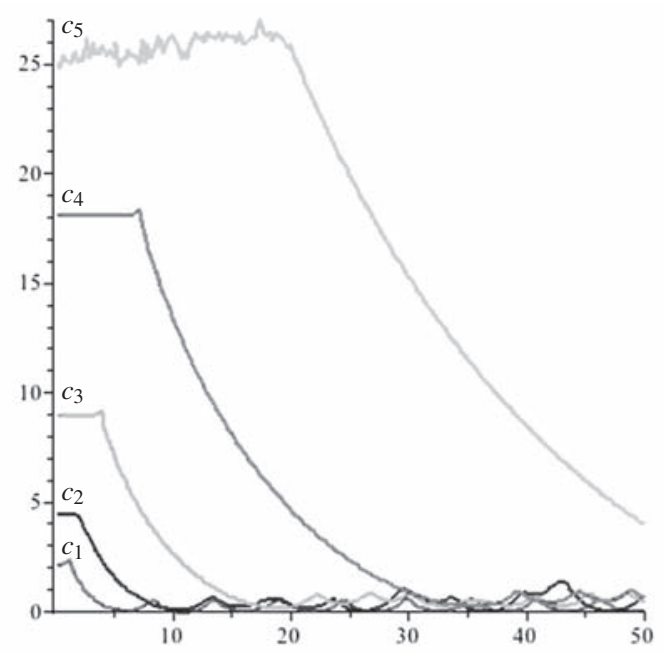

Figure 2.2. The number of significant digits, $-\log _{10}\left|Q_{v}[f]-I[f]\right|$, for $f(x)=(2+x)^{-1}, v=2\left(c_{1}\right), 4\left(c_{2}\right), 8\left(c_{3}\right), 16\left(c_{4}\right)$ and $32\left(c_{5}\right)$ for increasing $\omega$.
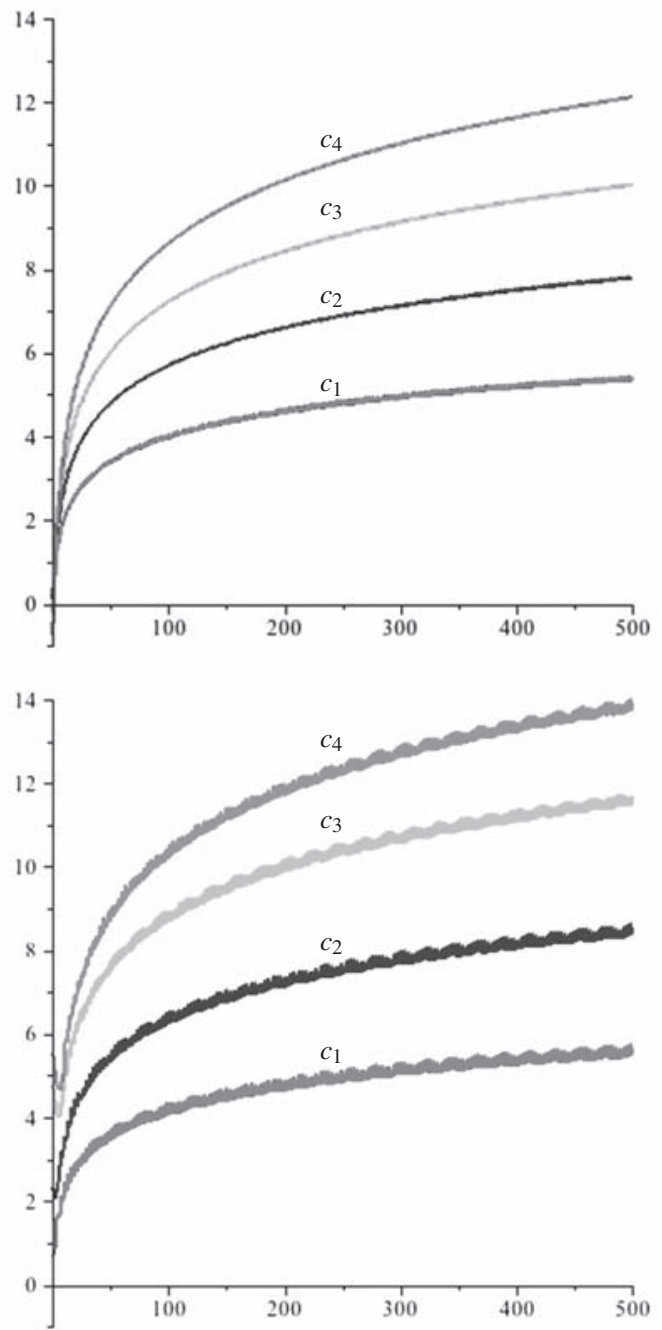

Figure 2.3. The number of significant digits once we use highly oscillatory quadrature. At the top the asymptotic method $A_{s}[f]$ for $s=0\left(c_{1}\right), 1\left(c_{2}\right), 2\left(c_{3}\right)$ and $3\left(c_{4}\right)$. At the bottom the Filon-type method $F_{c, m}[f]$ with $\boldsymbol{c}=[-1,1], \boldsymbol{m}=[1,1]\left(c_{1}\right), \boldsymbol{c}=[-1,0,1]$, $\boldsymbol{m}=[2,1,2]\left(c_{2}\right), \boldsymbol{c}=[-1,-1 / \sqrt{3}, 0,1 / \sqrt{3}, 1], \boldsymbol{m}=[3,1,1,1,3]$ $\left(c_{3}\right)$ and $c=[-1,-\sqrt{3 / 11}, 0, \sqrt{3 / 11}, 1], \boldsymbol{m}=[4,1,1,1,4]\left(c_{4}\right)$.

plicity $m_{k} \geq 1$, there exists a unique Hermite interpolating polynomial $p$ of degree $\sum m_{k}-1$ such that $p^{(i)}\left(c_{k}\right)=f^{(i)}\left(c_{k}\right)$, $i=0, \ldots, m_{k}-1, k=1, \ldots, v$. A Filon-type method consists of replacing $f$ by $p$ in the integral,

$$
F_{c, m}[f](\omega)=I[p](\omega) .
$$

Let $s=\min \left\{m_{1}, m_{v}\right\}$. It is trivial, substituting $F_{\boldsymbol{c}, \boldsymbol{m}}[f]-I[f]=$ $I[p-f]$ into $(2.2)$, to verify that the error is $O\left(\omega^{-s-2}\right)[9]$ : for large $\omega$ the behaviour is determined by asymptotics. On the other hand, once $\omega \rightarrow 0,(2.3)$ collapses into a quadrature method (using both the values of $f$ and of its derivatives) of the classical kind.

Beyond Filon-type methods. It is easy to extend the Filon method to integrals of the kind

$$
\int_{a_{-}}^{a_{+}} f(x) \mathrm{e}^{\mathrm{i} \omega g(x)} \mathrm{d} x,
$$

provided that $g^{\prime} \neq 0$ in $\left[a_{-}, a_{+}\right]$. More effort is required to deal with the case of stationary points $\xi \in\left[a_{-}, a_{+}\right]$where $g^{\prime}(\xi)=0$. In that case we need to interpolate to $f$ and a suitable number of derivatives at 
stationary points to recover the asymptotic behaviour of the 'plain' Filon-type method [9]. All this extends to a multivariate setting [10].

However, Filon-type methods are just one of the new breed of highly oscillatory quadrature methods, alongside Levin methods [ $[12$, 14] and the numerical stationary phase method [8]. The last word is a methodology which combines an insight from both Filon-type and stationary phase approaches in a true tour de force of asymptoticscum-numerics [7].

\section{Story 2: A highly oscillatory forcing term}

A good starting point is the differential equation for a nonlinear, frictionless pendulum which, in dimensionless form, reads $y^{\prime \prime}+\sin y=0, y(0)=y_{0}, y^{\prime}(0)=y_{0}^{\prime}$. This is a Hamiltonian system in a single variable and its dynamics is as simple as they come: the origin is a centre, surrounded in the phase plane by stable periodic orbits. Suppose, however, that we impart rapid oscillations to the base of the pendulum: the outcome is the non-autonomous equation

$$
y^{\prime \prime}+\sin y=c \cos \omega t, \quad t \geq 0, \quad y(0)=y_{0}, \quad y^{\prime}(0)=y_{0}^{\prime},
$$

where $\omega \gg 1$. What can we expect from the solutions of (3.4)? Fig. 3.4 displays a trajectory corresponding to $y_{0}=2$, $y_{0}^{\prime}=0$ and $c=1$ in the phase plane for $\omega=5,10$ and 20. We can discern a thick curve in the background: this is the corresponding trajectory of the nonlinear pendulum $y^{\prime \prime}+\sin y=0$ and it is evident that the trajectory of the forced equation winds round and round the periodic trajectory of the unforced equation. Surprisingly, the amplitude of this winding motion decreases with $\omega$. (This is the reason why we have used in Fig. 3.4 such modest values of $\omega$ : with much greater values the winding motion would have been invisible to the naked eye.)

This effect of growing $\omega$ is important: rapid oscillation of the forcing term leads to an increasingly smaller perturbation and it stabilises the motion. By the same token, slowing down the oscillation destabilises the motion: try the same computation with $\omega=2$. There is an important lesson here: high oscillation is good for dynamics while, at the same time, being lethal for naive numerics. True to our paradigm, instead of relying on the (local) Taylor series, we seek an asymptotic expansion.

It is convenient to consider a broader framework: following [3], we examine the equation

$y^{\prime \prime}+f(y)=\sum_{m=-\infty}^{\infty} a_{m}(t) \mathrm{e}^{\mathrm{i} m \omega t}, \quad t \geq 0, \quad y(0)=y_{0}, \quad y^{\prime}(0)=y_{0}^{\prime}$,

where $f$ and $a_{m}, m \in \mathbb{Z}$, are analytic functions and $\sum_{m}\left|a_{m}(t)\right|<$ $\infty, t \geq 0$. Our claim is that we can expand the solution in the form

$$
y(t) \sim p_{0,0}(t)+\sum_{r=2}^{\infty} \frac{1}{\omega^{r}} \sum_{m=-\infty}^{\infty} p_{r, m}(t) \mathrm{e}^{\mathrm{i} m \omega t}, \quad t \geq 0,
$$

where the functions $p_{r, m}$, which are independent of $\omega$, can be obtained explicitly in a recursive manner.

In principle, we need to expand everything in (3.5) in asymptotic series. This is fairly easy for $y^{\prime \prime}(t)$ but much more challenging (and messy) for $f(y(t))$ and, at the conclusion of all this algebra galore, we have an expansion in two scales: orders of magnitude $\omega^{-r}$ and oscillators $\mathrm{e}^{\mathrm{i} m \omega t}$. We commence by

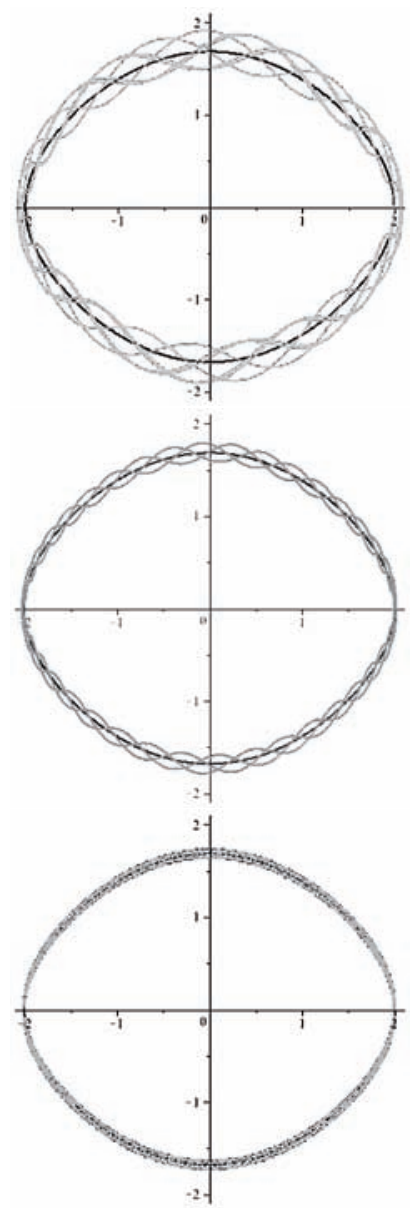

Figure 3.4. A phase-plane trajectory of the solution of $y^{\prime \prime}+\sin y=$ $\cos \omega t, y(0)=3, y^{\prime}(0)=0$, with $\omega=5,10,20$. The black curve is the corresponding periodic trajectory of $y^{\prime \prime}+\sin y=0$.

separating the expansion into orders of magnitude. For $r=0$ we have

$$
p_{0,0}^{\prime \prime}-\sum_{m=-\infty}^{\infty} m^{2} p_{2, m} \mathrm{e}^{\mathrm{i} m \omega t}+f\left(p_{0,0}\right)=\sum_{m=-\infty}^{\infty} a_{m} \mathrm{e}^{\mathrm{i} m \omega t}
$$

and, separating oscillators, we have

$$
\begin{aligned}
p_{0,0}^{\prime \prime}+f\left(p_{0,0}\right) & =0, \quad t \geq 0, \quad p_{0,0}(0)=y_{0}, \quad p_{0,0}^{\prime}(0)=y_{0}^{\prime}, \\
p_{2, m} & =-\frac{a_{m}}{m^{2}}, \quad m \neq 0 .
\end{aligned}
$$

Note that we have imposed on $p_{0,0}$ the same initial conditions as on $y$; this means that we need to impose $\sum_{m} p_{r, m}(0)=$ $\sum_{m} p_{r, m}^{\prime}(0)=0$ in the sequel.

Next, over to $r=1$,

$$
\sum_{m=-\infty}^{\infty}\left(2 \mathrm{i} m p_{2, m}^{\prime}-m^{2} p_{3, m}\right) \mathrm{e}^{\mathrm{i} m \omega t}=0
$$

and, separating scales,

$$
p_{3, m}=\frac{2 \mathrm{i}}{m} p_{2, m}^{\prime}=-\frac{2 \mathrm{i}}{m^{3}} a_{m}^{\prime}, \quad m \neq 0 .
$$

Likewise, $r=2$ results in

$$
\sum_{m=-\infty}^{\infty}\left(p_{2, m}^{\prime \prime}-2 \mathrm{i} m p_{3, m}^{\prime}-m^{2} p_{4, m}\right) \mathrm{e}^{\mathrm{i} m \omega t}+f^{\prime}\left(p_{0,0}\right) \sum_{m=-\infty}^{\infty} p_{2, m} \mathrm{e}^{\mathrm{i} m \omega t}=0,
$$



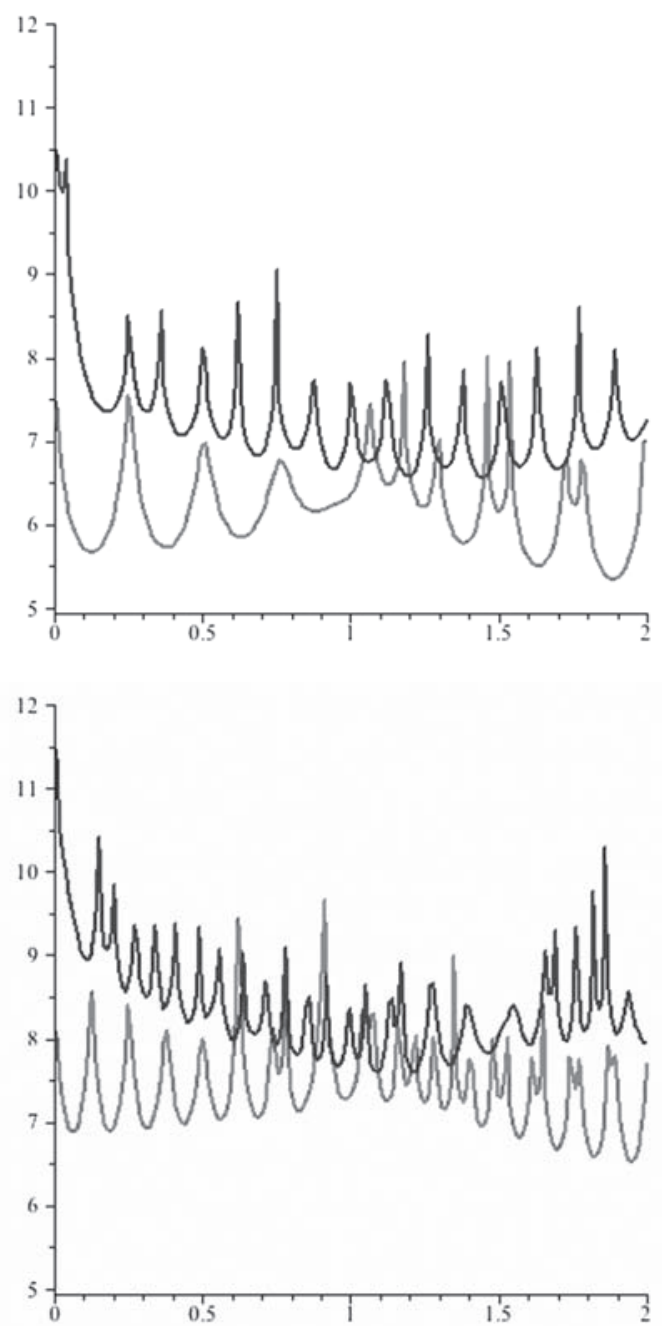

Figure 3.5. The error $-\log _{10}\left|y^{[s]}(t)-y(t)\right|$ for the forced nonlinear pendulum (3.4): $\omega=25$ at the top and $\omega=50$ at the bottom, $s=2$ in gray and $s=4$ in black.

therefore

$$
\begin{aligned}
& p_{2,0}^{\prime \prime}+f^{\prime}\left(p_{0,0}\right) p_{2,0}=0, \quad t \geq 0, \sum_{m=-\infty}^{\infty} p_{2, m}(0)=\sum_{m=-\infty}^{\infty} p_{2, m}^{\prime}(0)=0, \\
& p_{4, m}=\frac{1}{m^{2}}\left[p_{2, m}^{\prime \prime}-2 \mathrm{i} m p_{3, m}^{\prime}+f^{\prime}\left(p_{0,0}\right) p_{2, m}\right], \\
& m \neq 0 .
\end{aligned}
$$

A general rule emerges: for each order of magnitude $r$ we obtain $p_{r, 0}$ solving a differential equation and $p_{r+2, m}, m \neq 0$, by recursion. These differential equations need to be solved numerically but this is not a problem since they are all nonoscillatory: the solution becomes oscillatory only once we assemble it into an appropriately truncated expansion (3.6),

$$
y^{[s]}(t)=p_{0,0}(t)+\sum_{r=2}^{s} \frac{1}{\omega^{r}} \sum_{m=-\infty}^{\infty} p_{r, m}(t) \mathrm{e}^{\mathrm{i} m \omega t}, \quad t \geq 0 .
$$

All relevant equations can be derived explicitly [3].

Specialising to the forced nonlinear pendulum (3.4), we use (3.4-6) and, for good measure, consider also $r=3$, 4. We

$$
\begin{aligned}
& \text { have } p_{3, m} \equiv 0, \\
& p_{0,0}^{\prime \prime}+\sin p_{0,0}=0, \quad t \geq 0, \\
& \quad p_{0,0}(0)=2, \quad p_{0,0}^{\prime}(0)=0, \\
& p_{2,0}^{\prime \prime}+\left(\cos p_{0,0}\right) p_{2,0}=0, \quad t \geq 0, \\
& p_{2,0}(0)=1, \quad p_{2,0}^{\prime}(0)=0, \\
& p_{4,0}^{\prime \prime}+\left(\cos p_{0,0}\right) p_{4,0}=\frac{1}{4}\left(1+2 p_{0,0}^{2}\right) \sin p_{0,0}, \\
& p_{4,0}(0)=\cos 2, \quad p_{4,0}^{\prime}(0)=0,
\end{aligned}
$$

and

$$
\begin{aligned}
y^{[2]}(t)=p_{0,0}(t) & +\frac{1}{\omega^{2}}\left[p_{2,0}(t)-\cos \omega t\right], \\
y^{[4]}(t)=p_{0,0}(t) & +\frac{1}{\omega^{2}}\left[p_{2,0}(t)-\cos \omega t\right] \\
& +\frac{1}{\omega^{4}}\left[p_{4,0}(t)-\cos \omega t \cos p_{0,0}(t)\right] .
\end{aligned}
$$

Fig. 3.5 displays the number of significant digits recovered by $y^{[2]}$ and $y^{[4]}$, respectively, for $\omega=25$ and $\omega=50$. All is in line with the theory and if the accuracy is insufficient for you - well, just take larger $\omega \ldots$

Beyond highly oscillatory forced ODEs. Equation (3.5) is but one example of ordinary differential equations with highly oscillatory forcing which can be expanded by this synthesis of asymptotic and numerical reasoning. With greater generality, it is possible to extend this analysis to ODE systems

$$
\boldsymbol{y}^{\prime}=\boldsymbol{f}(\boldsymbol{y})+\sum_{m=-\infty}^{\infty} \boldsymbol{a}_{m}(t) \mathrm{e}^{\mathrm{i} m \omega t}, \quad t \geq 0, \quad \boldsymbol{y}(0)=\boldsymbol{y}_{0}
$$

$[4,15]$ and beyond. Equations of this kind are important in many applications, e.g., in electronics, where oscillation is generated by high-frequency input.

A more general framework is provided by the heterogeneous multi scale method [1] and it allows not just highly oscillatory forcing terms but also highly oscillatory coefficients, but the quid pro quo is that expansions can be practically generated up to $O\left(\omega^{-r}\right)$ only for fairly modest values of $r \geq 1$.

\section{Story 3: The linear Schrödinger equation}

The linear Schrödinger equation

$$
\partial_{t} u=\mathrm{i} \varepsilon \partial_{x}^{2} u+\mathrm{i} \varepsilon^{-1} V(x) u, \quad t \geq 0, \quad-1 \leq x \leq 1,
$$

where both the initial value $u(\cdot, 0)$ and the interaction potential $V(x)$ are smooth and periodic, describes the quantum state of a single particle: essentially, it is the equivalent of Newton's law in a quantum setting. The parameter $\varepsilon>0$ is very small, rendering the solution of this deceptively simple linear PDE fairly knotty.

Standard numerical wisdom goes along the following lines: we commence by discretising (4.10) in some $M$-dimensional space, e.g., by replacing $\partial_{x}^{2} u$ by a linear combination of function values along a grid (finite differences) or moving to Fourier space and considering there an $\mathrm{L}_{2}$ projection on $N$ th degree trigonometric polynomials, $M=2 N+1$ (a spectral method). The outcome is a set of linear ODEs of the form

$$
\boldsymbol{u}^{\prime}=\mathrm{i}\left(\varepsilon \mathcal{K}+\varepsilon^{-1} \mathcal{D}_{V}\right) \boldsymbol{u}, \quad t \geq 0, \quad \boldsymbol{u}(0)=\boldsymbol{u}_{0},
$$

where $\mathcal{K}$ and $\mathcal{D}_{V}$ are matrices corresponding to approximate differentiation and multiplication by $V$, respectively. Solving 
(4.11) should be easy in principle; after all an explicit solution is available,

$$
\boldsymbol{u}(t)=\exp \left(\mathrm{i} t\left(\varepsilon \mathcal{K}+\varepsilon^{-1} \mathcal{D}_{V}\right)\right) \boldsymbol{u}_{0}, \quad t \geq 0
$$

except that the tiny $\varepsilon$ spoils the party. The dimension $M$ is large (and our real interest is in considering (4.10) in a multivariate setting: the dimension soon becomes really large!) and the only realistic hope of computing the matrix exponential with reasonable accuracy is by Krylov subspace methods [6]: we replace the computation of a 'big' exponential by a (hopefully) small, $m \times m$, one, at a cost of $O\left(m^{2} M\right)$ operations. Except that our hope is in vain: once $\varepsilon$ is very small, $m$ becomes large, $m=O(M)$, and the overall cost, $O\left(M^{3}\right)$, is unacceptably large.

The good news is that a ready alternative is available: an exponential splitting. In its simplest manifestation, the Strang splitting

$$
\mathrm{e}^{\mathrm{i} t\left(\varepsilon \mathcal{K}+\varepsilon^{-1} \mathcal{D}_{V}\right)} \approx \mathrm{e}^{\frac{1}{2} \mathrm{i} t \varepsilon \mathcal{K}} \mathrm{e}^{\mathrm{i} t \varepsilon^{-1}} \mathcal{D}_{V} \mathrm{e}^{\frac{1}{2} \mathrm{i} t \varepsilon \mathcal{K}}
$$

incurs an error of $O\left(t^{3}\right)$ and is easy to evaluate: e.g., once we use a spectral method, $\mathcal{K}$ is a diagonal matrix and $\mathcal{D}_{V}$ a circulant, whose exponential can be evaluated with two Fast Fourier Transforms (FFT) [11]. Unfortunately, the error $O\left(t^{3}\right)$ of the Strang splitting is unacceptably large.

It is possible to design higher-order splittings, e.g.

$$
\mathrm{e}^{\frac{1}{2} \alpha t \mathcal{B}} \mathrm{e}^{\alpha t \mathcal{A}} \mathrm{e}^{\frac{1}{2}(1-\alpha) t \mathcal{B}} \mathrm{e}^{(1-2 \alpha) t \mathcal{A}} \mathrm{e}^{\frac{1}{2}(1-\alpha) t \mathcal{B}} \mathrm{e}^{\alpha t \mathcal{A}} \mathrm{e}^{\frac{1}{2} \alpha t \mathcal{B}}
$$

where $\mathcal{B}=\mathrm{i} \varepsilon \mathcal{K}, \mathcal{A}=\mathrm{i} \varepsilon^{-1} \mathcal{D}_{V}$ and $\alpha=(2-\sqrt[3]{2})^{-1}$ has error of $O\left(t^{5}\right)$. In general, we can get $O\left(t^{2 n+1}\right)$ with $2 \cdot 3^{n-1}+1$ terms but this is simply much too much for practical computation with large $n$, even though each individual exponential is cheap. Moreover, note that all these are expansions in the step size $t$ but we have two other small quantities, $\varepsilon$ and $M^{-1}$ : a good expansion should take them all into account.

By this stage of our narrative, a remedy should stare us in the face: an asymptotic expansion! Following upon the ideas in [2], we thus assume that $t \sim O\left(\varepsilon^{1 / 2}\right), M \sim O\left(\varepsilon^{-1 / 2}\right)$ and seek an expansion of the form

$$
\mathrm{e}^{\mathcal{R}_{0}} \mathrm{e}^{\mathcal{R}_{1}} \cdots \mathrm{e}^{\mathcal{R}_{s}} \mathrm{e}^{\mathcal{T}_{s+1}} \mathrm{e}^{\mathcal{R}_{s}} \cdots \mathrm{e}^{\mathcal{R}_{1}} \mathrm{e}^{\mathcal{R}_{0}}
$$

where $\mathcal{R}_{0}=O\left(\varepsilon^{-1 / 2}\right), \mathcal{R}_{1}=O\left(\varepsilon^{1 / 2}\right), \ldots, \mathcal{R}_{s}=O\left(\varepsilon^{s-1 / 2}\right)$ and $\mathcal{T}_{s}=O\left(\varepsilon^{s+1 / 2}\right)$ : an asymptotic splitting.

An important observation with regard to $(4.3-5)$ is that they are all palindromic: the same whether we read them from the left or from the right. This has a number of advantages. This time symmetry is good in minimising the number of terms because all expansions are in odd powers of the small parameter. Moreover, since both $\mathrm{i} \partial_{x}^{2}$ and multiplication by iV are skew-Hermitian operators, the solution operator of (4.10) is unitary and palindromy makes it easier to respect this feature under discretisation.

The right approach towards computing the asymptotic splitting (4.14) is to abandon the semi-discretisation (4.11) for the time being and split the formal solution operator, $\exp \left(\mathrm{i} t\left(\varepsilon \partial_{x}^{2}+\varepsilon^{-1} V\right)\right)$. Only once we have done so, we replace everything with a finite-dimensional approximation. Our main weapon is the symmetric Baker-Campbell-Hausdorff ( $s B C H)$ formula

$$
\mathrm{e}^{\frac{1}{2} X} \mathrm{e}^{Y} \mathrm{e}^{\frac{1}{2} X}=\mathrm{e}^{\mathrm{sBCH}(X, Y)}
$$

where

$$
\begin{aligned}
\operatorname{sBCH}(t X, t Y)= & t(X+Y)-t^{3}\left(\frac{1}{24}[[Y, X], X]+\frac{1}{12}[[Y, X], Y]\right) \\
+ & t^{5}\left(\frac{7}{5760}[[[[Y, X], X], X], X]\right. \\
& +\frac{7}{1440}[[[[Y, X], X], X], Y] \\
& +\frac{1}{180}[[[[Y, X], X], Y], Y] \\
& +\frac{1}{720}[[[[Y, X], Y], Y], Y] \\
& \left.+\frac{1}{480}[[[Y, X], X],[Y, X]]\right)+O\left(t^{7}\right) .
\end{aligned}
$$

Note that the expansion is in odd powers of $t$, reaping a benefit of palindromy!

We let $\tau=\mathrm{i} \Delta t$, where $\Delta t$ is the time step. The algorithm commences by setting $\mathcal{T}_{0}=\tau \varepsilon^{-1} V+\tau \varepsilon \partial_{x}^{2}$ (the term we wish to split) and $\mathcal{R}_{0}=\frac{1}{2} \tau \varepsilon^{-1} V$ (half the asymptotically larger term, which we wish to knock out by this stage), therefore

$$
\mathrm{e}^{\tau \varepsilon^{-1} V+\tau \varepsilon \partial_{x}^{2}}=\mathrm{e}^{\mathcal{T}_{0}}=\mathrm{e}^{\mathcal{R}_{0}} \mathrm{e}^{\mathrm{sBCH}\left(-2 \mathcal{R}_{0}, \mathcal{T}_{0}\right)} \mathrm{e}^{\mathcal{R}_{0}} .
$$

Note that, having just 'decapitated' the leading term, $\mathcal{T}_{1}:=$ $\operatorname{sBCH}\left(-2 \mathcal{R}_{0}, \mathcal{T}_{0}\right)=O\left(\varepsilon^{1 / 2}\right)$, we next identify the leading (i.e. $O\left(\varepsilon^{1 / 2}\right)$ ) term of $\mathcal{T}_{1}$ and let $\mathcal{R}_{1}$ be half of it. Therefore

$$
\mathrm{e}^{\tau \varepsilon^{-1} V+\tau \varepsilon \partial_{x}^{2}}=\mathrm{e}^{\mathcal{R}_{0}} \mathrm{e}^{\mathcal{R}_{1}} \mathrm{e}^{\mathrm{sBCH}\left(-2 \mathcal{R}_{1}, \mathcal{T}_{1}\right)} \mathrm{e}^{\mathcal{R}_{1}} \mathrm{e}^{\mathcal{R}_{0}}
$$

and $\operatorname{sBCH}\left(-2 \mathcal{R}_{1}, \mathcal{T}_{1}\right)=O\left(\varepsilon^{3 / 2}\right)$. We continue in this vein until the right accuracy has been attained, except that, more perhaps than elsewhere, the devil is in the detail! On the face of it, the $\mathrm{sBCH}$ formula introduces commutators galore and this might mire the entire enterprise in expensive calculations. Except that, upon further examination, commutators go away! For example, it is easy to confirm that $\left[V, \partial_{x}^{2}\right]=-V^{\prime \prime}-2 V^{\prime} \partial_{x}$. In general, every combination of nested commutators of $V$ and $\partial_{x}^{2}$ can be written in the form $\sum_{k=0}^{n} y_{k}(x) \partial_{x}^{k}$ for some $n \geq 0$ and functions $y_{1}, \ldots, y_{n}$ which depend on $V$ and its derivatives.

Commutators go away but another problem rears its head: odd powers of $\partial_{x}$, which may cause instability and play havoc with our expansions. The remedy is to get rid of odd derivatives by a simple trick, e.g.

$$
y(x) \partial_{x}=-\frac{1}{2} \int_{x_{0}}^{x} y(\xi) \mathrm{d} \xi \partial_{x}^{2}-\frac{1}{2} y^{\prime}(x)+\frac{1}{2} \partial_{x}^{2}\left[\int_{x_{0}}^{x} y(\xi) \mathrm{d} \xi\right] .
$$

We have only even-order derivative operators on the right and all is well! The outcome,

$\mathcal{R}_{0}=\frac{1}{2} \tau \varepsilon^{-1} V=O\left(\varepsilon^{-1 / 2}\right)$,

$\mathcal{R}_{1}=\frac{1}{2} \tau \varepsilon \partial_{x}^{2}+\frac{1}{24} \tau^{3} \varepsilon^{-1} V^{\prime \prime}=O\left(\varepsilon^{1 / 2}\right)$,

$\mathcal{R}_{2}=-\frac{1}{12} \tau^{3} \varepsilon\left\{\partial_{x}^{2}\left(V^{\prime \prime} \cdot\right)+V^{\prime \prime} \partial_{x}^{2}\right\}+\frac{1}{120} \tau^{5} \varepsilon^{-1} V^{\prime \prime} V^{2}=O\left(\varepsilon^{3 / 2}\right)$,

etc. is our asymptotic splitting.

Now - and only now - we discretise derivatives by one of the very powerful methods of numerical analysis, e.g., spectral collocation [5]. All this procedure can be further improved by, paradoxically, first knocking out the smaller term $\tau \varepsilon \partial_{x}^{2}$ since this counterintuitive step means that all $O\left(\varepsilon^{m / 2}\right)$ exponentials for $m \leq \frac{1}{2}$ can be evaluated easily either because they are diagonal or by FFT. The remaining exponentials can be reduced by Krylov subspace methods to a very small number of dimensions, e.g., for $s=2$ we need just dimension 3 for $\mathcal{R}_{2}$ and dimension 2 for $\mathcal{T}_{3}$. 
Beyond univariate Schrödinger. Equation (4.10) is the simplest model of linear Schrödinger equations in quantum chemistry. In general, we wish to solve the multivariate equation $\partial_{t} u=\mathrm{i} \varepsilon \nabla^{2} u+$ $\mathrm{i} \varepsilon^{-1} V(\boldsymbol{x}) u$ in a $d$-dimensional torus. Provided that $d$ is moderately small, the asymptotic expansion (4.14) generalises and, at the cost of $O\left(N^{d} \log N\right)$ operations, remains practically feasible. However, once $d$ is large, this approach must be matched by specialised methodologies for highly-dimensional setting, e.g., sparse grids: this is under active research. Another generalisation is to time-dependent interaction potentials, important if magnetism effects are taken on board.

Yet, the potential scope of 'asymptotic splitting' is much wider, not just because of the asymptotic rate of decay but because commutators are replaced by easy-to-evaluate expressions. Only future can tell the limits of this methodology.

\section{Bibliography}

[1] A. Abdulle, W. E, B. Engquist, and E. Vanden-Eijnden. The heterogeneous multiscale method. Acta Numerica, 21:1-87, 2012.

[2] P. Bader, A. Iserles, K. Kropielnicka, and P. Singh. Effective approximation for the linear time-dependent Schrödinger equation. Technical Report NA2012/05, DAMTP, University of Cambridge, 2012.

[3] M. Condon, A. Deaño, and A. Iserles. On second order differential equations with highly oscillatory forcing terms. Proc. Royal Soc. A, 466:1809-1828, 2010.

[4] M. Condon, A. Deaño, and A. Iserles. On systems of differential equations with extrinsic oscillations. Discr. $\mathcal{E}$ Cont. Dynamical Sys., 28:1345-1367, 2010.

[5] J. S. Hesthaven, S. Gottlieb, and D. Gottlieb. Spectral Methods for Time-Dependent Problems. Cambridge University Press, Cambridge, 2007.

[6] M. Hochbruck and C. Lubich. On Krylov subspace approximations to the matrix exponential operator. SIAM J. Numer. Anal., 34:1911-1925, 1997.

[7] D. Huybrechs and S. Olver. Superinterpolation in highly oscillatory quadrature. Found. Comp. Maths, 12:203-228, 2012.
[8] D. Huybrechs and S. Vandewalle. On the evaluation of highly oscillatory integrals by analytic continuation. SIAM J. Numer. Anal., 44:1026-1048, 2006.

[9] A. Iserles and S. P. Nørsett. Efficient quadrature of highly oscillatory integrals using derivatives. Proc. Royal Soc. A, 461:1383-1399, 2005.

[10] A. Iserles and S. P. Nørsett. Quadrature methods for multivariate highly oscillatory integrals using derivatives. Maths Comp., 75:1233-1258, 2006.

[11] S. Jin, P. Markowich, and Ch. Sparber. Mathematical and computational methods for semiclassical Schrödinger equations. Acta Numerica, 20:121-210, 2011.

[12] D. Levin. Fast integration of rapidly oscillatory functions. $J$. Comput. Appl. Maths, 67:95-101, 1996.

[13] F. W. J. Olver. Asymptotics and Special Functions. A K Peters Ltd, Wellesley, MA, 1997.

[14] S. Olver. Moment-free numerical integration of highly oscillatory functions. IMA J. Numer. Anal., 26:213-227, 2006.

[15] J. M. Sanz-Serna. Modulated Fourier expansions and heterogeneous multiscale methods. IMA J. Numer. Anal., 29:595605,2009 .

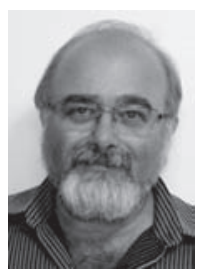

Arieh Iserles [a.iserles@damtp.cam.ac.uk] is professor of the numerical analysis of differential equations at the University of Cambridge. He was awarded in 1999 the Lars Onsager Medal and in 2012 the David Crighton Medal and was an invited speaker at the 6th European Congress of Mathematicians. He is Managing Editor of Acta Numerica, Editor-in-Chief of the IMA Journal of Numerical Analysis and was the Chair of the Society for Foundations of Computational Mathematics. His interests comprise numerical analysis of differential equations, approximation theory, computational dynamics and the theory of differential and functional equations.

\title{
Touching the Abstract: Mathematics at the Museum
}

\author{
Enrico Giusti (II Giardino di Archimede, Firenze, Italy)
}

A visitor venturing into the mathematics section of a modern science museum could happen on one of the most interesting, stimulating, surprising mechanical phenomena: a Lorenz chaotic water wheel. Many of you will be familiar with it: water flows into containers that hang on the edge of a rotating circle and slowly leaks from a hole in the bottom. In the beginning, the movement is rather regular and predictable but soon, either because some visitor sneezes or because the jet is not perfectly uniform or because some butterfly flaps its wings in the Amazon jungle, the movement becomes essentially irregular: even a powerful computer cannot predict it for more than a few minutes in advance. In essence, it's a bril- liant example of deterministic chaos - a process in which unavoidable perturbations in the environment multiply so quickly that a medium-term forecast is impossible. Still, modern mathematics is able to explain these phenomena: the fountain is a physical model of the power of mathematics and an example of its success.

Science museums haven't always had such spectacular mathematics sections. On the contrary, the oldest ones, especially, seemed to want to reproduce the pedantic approach of school learning: portraits of famous mathematicians of the past hung on the walls of the room; threedimensional models in display cases showed the interests of 19th century mathematicians; and all around the room 
were written thousands of decimals of $\pi$, of which rumour had it that the three thousand seven hundred and fortythird figure was wrong.

But this belongs to the past. Today, one can see beautiful moving images and computer experiments, admire the random movements of discs simulating Brownian motion, and bounce balls that will end up in a Gaussian curve. The visitor who walks through the room of mathematics will emerge amazed and amused.

But once the wonder and marvel are gone, if our visitor asks himself: "Where is the mathematics?" then what can he answer? Behind the bells and whistles of a modern museum, just as behind the pedantry of the older one, mathematics is often evoked but seldom seen. It's somewhere else. Or rather - mathematics remains inside the object that we are looking at or the mechanism that we interact with, unable to come to the surface and show itself. It just stays buried: from the point of view of communication it is completely dead.

Mind you - when I say that this mathematics is "dead", I don't mean that it's obsolete, or bad, or irrelevant: quite the opposite. The theory of chaos of our example is one of the most advanced and promising fields of modern mathematics. The problem is that this mathematics does not emerge from the physical objects that contain it; it has done its job elsewhere, in another context, and the mechanism on display does not show it any more.

Instead, we want a 'living' mathematics, a mathematics caught in the moment it operates, that determines the behaviour of the machines we use and the phenomena we observe. One of the big challenges in engineering and building a museum is to make the displayed objects show mathematics in action.

Here is another example that can help us understand the difference between living and dead mathematics better. This time let's leave modern mathematics and let's look at one of the oldest theorems, one that most people are familiar with: good old Pythagoras' theorem.

You have probably seen somewhere a "hydraulic" proof of this theorem. The squares of the legs are filled with water, while the one of the hypotenuse is empty. When turning the figure, the square of the hypotenuse goes down and the ones of the legs go up. The water flows through tubes until the square of the hypotenuse is filled and the squares of the legs are empty, which shows that their sum is equal to the first.

Another very simple proof involves a large square, the side of which is equal to the sum of the two legs.

The relevant right triangle is in one corner, and identical ones are placed on the other corners. The area that remains is the square of the hypotenuse, so the large square is equal to the square of the hypotenuse plus four triangles. Let's now move the triangles in order to form two rectangles; what remain are the two squares of the legs so in this configuration the large square (which was equal to the square of the hypotenuse plus four triangles) is now equal to the same four triangles plus the squares of the legs. Q.E.D.

The first proof, which is based on filling either the squares of the legs or the square of the hypotenuse, is a perfect example of dead mathematics. In fact, mathemat-
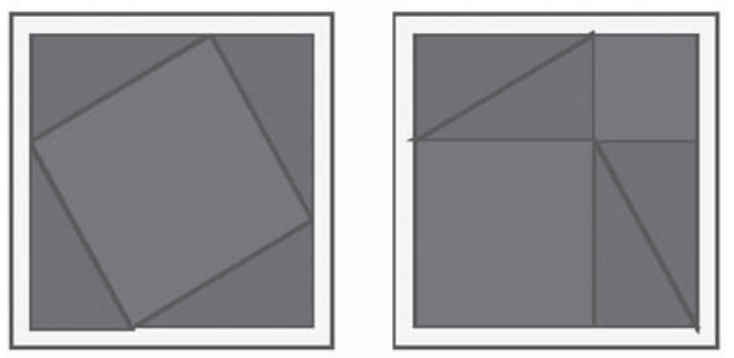

ics was sometimes used while proving the theorem but there is no trace of this proof in the experiment. This hydraulic test is based on a number of assumptions that have nothing to do with mathematics: that the containers are actually square; that their thickness is uniform; that there are no hidden parts where the water could go; and that the vessels are in fact precisely filled. Even if these assumptions are all reasonable and natural, we can only trust in a visual impression, since there is no logical reasoning that can assure us that this is in fact the case and that the appearance of areas being the same is not a trick. Moreover, even if the object on display actually shows that the square of the hypotenuse is equal to the sum of the squares of the two legs, nothing proves that a different right-angled triangle would possess the same property. In essence, the hydraulic device gives a verification without mathematics.

The second proof is completely different because it speaks not to the eye but to the mind. It's not even important that the four triangles are absolutely identical, that their sides be perfectly straight or that their right angle be exactly right. As with the figures we draw in geometry, we just need the objects to be reasonably precise, so that they can guide reasoning while avoiding glaring errors. Once we assume that they are indeed right-angled, identical triangles, then the quadrilateral of the first figure is a square, just like those of the second figure, whose sum is equal to the first. What is more, although the specific object by necessity only describes a particular case, it's easy to understand that the same reasoning will be valid for any right-angled triangle. All this is possible because it is in fact a reasoning and not an empirical verification with no mathematical content. In brief, while the hydraulic device displays dead mathematics, the second proof shows mathematics in its making - living, active mathematics.

Live mathematics has another characteristic that distinguishes it from its dead counterpart: it can be read on more than one level. The lowest level consists of the visual verification that the uncovered parts of the large square are, on the one hand, the square of the hypotenuse and, on the other, the squares of the legs of our right-angled triangle.

A level immediately above would prove, for example, that the first quadrilateral is in fact a square, that is, that its angles are right angles.

A third level of interpretation may consist of the remark that the proof of the last assertion depends on the fact that the sum of the angles of a triangle is equal to two right angles. This theorem can only work if the 
parallel postulate is assumed: in fact, it is equivalent to this postulate. Consequently, Pythagoras' theorem is a theorem within Euclidean geometry and it does not hold in non-Euclidean geometries.

But where is the living mathematics? One answer is: in the proofs. Proofs are the salt and the essence of mathematics and it is therefore obvious that the mathematics they contain is alive and kicking. On the other hand, the proofs that can materialise in physical objects are too few for us to construct an entire museum out of them. And, by the way, who would be interested in a museum made of proofs? We must find new roads, other manifestations of mathematics in action.

Luckily there's a lot more mathematics around than one would normally think and, if we look all around ourselves, we can find objects and mechanisms that show how much it is part of everyday life. Let's look at an example.
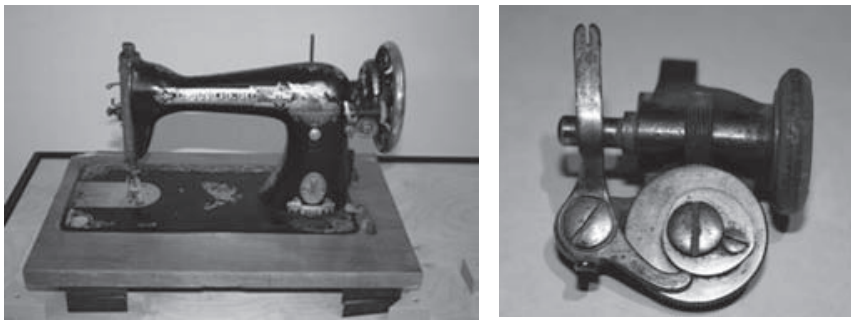

This is an old sewing machine. But the part that we are interested in is just the small piece shown in the picture next to it.

This small object regulates the coiling of the thread around the spool. In order to coil the thread, the spool has to be turned and at the same time it must be moved back and forth so that it is filled uniformly. This is why it is necessary that this alternating movement be as uniform as possible, otherwise the thread would accumulate more in the areas in which the spool slows down and less in the ones where it speeds up. Normally, when something changes direction, it slows to a stop and then starts again in the opposite direction. Here instead we want speed to remain the same even when the direction changes. How do we get an oscillation at a constant velocity?

To answer this question, let's leave the sewing machine for a moment and let's move back some 22 centuries, to the days of Archimedes of Syracuse. Archimedes is still famous today for a number of discoveries and a few oddities but we are interested in a specific curve that he introduced and studied, and that bears his name: the spiral.

It's very easy to draw a spiral of Archimedes. Rotate a disc (at constant velocity) and at the same time trace a line from the centre of the disc towards its circumference, also at constant velocity. The resulting line is the spiral of Archimedes.

Conversely, a point that remains on a spiral while the disc is rotating will move from the centre to the circumference at constant velocity. We can build a mechanism to show this property. The heart-shaped curve is composed of two arcs of an Archimedes spiral, and it is attached to the black disc. When this starts moving clockwise, the point of the vertical shaft always remains in contact with

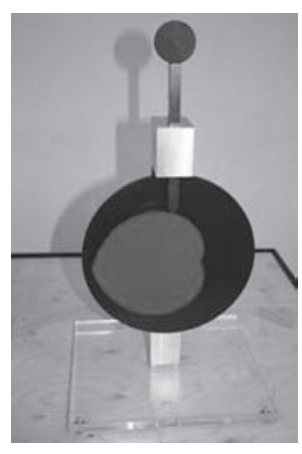

the spiral, so the whole shaft moves up at constant velocity. Of course, this happens only until it gets to the point of the heart. Then it goes onto the second spiral arc which has the opposite orientation, and the shaft starts descending at the same speed, until it reaches the first arc and starts going up again, and so on.

The properties of the spiral of Archimedes allowed us to create what we needed: an oscillation that takes place at constant velocity. The same mechanism regulates the spooling of the thread: the heart-shaped figure, composed of two arcs of spiral, coils the thread onto the spool in a uniform manner, with no bulking in some points and thinning in others.

Now we can better understand how mathematics can be made to emerge from objects. A physical object never has a mathematical content in itself, even if its functioning depends on, and is determined by, the underlying mathematics. In order for the latter to emerge it is necessary that this object is not in isolation but is part of a sequence, of a path which illustrates its many aspects. In particular, the mathematical object inside it needs to be made visible, by showing its relevant properties and connecting it to the visible object. Without the first aspect, that is, without the illustration of the mathematical reasons that lie behind its functioning, the mechanism for spooling the thread can show nothing more than dead mathematics, evoked but not there. Conversely, without a physical object to relate to, the spiral, its construction and its mathematical properties would remain confined in a separate and in some ways alien universe. In this case, mathematics becomes a self-referential game, which can at times be intellectually stimulating but rarely engages the public at large. When placed within a path and linked by an intermediate mechanism (which is not entirely mathematical because it has an obvious physical reality but not completely real because it was constructed only with the purpose of illustrating a mathematical principle) the two worlds become linked and interdependent. The spiral of Archimedes, in action, causes the appropriate coiling of the spool.

A similar reasoning can be made on the cycloid. As we know, the cycloid is the curve generated by a point on a circumference that rolls along a straight line.

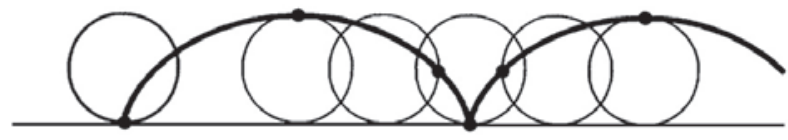

Among its many properties is that it is an isochronal curve. All the oscillations of a body falling along an inverted cycloid have a constant duration, irrespective of their amplitude. This property was utilised by Christiaan Huygens in the 17th century to build a pendulum clock. By making the weight oscillate on a cycloid, the duration of each oscillation was always the same, for example one 
per second, so the clock was exact. The technical problem was to get the pendulum to describe a cycloid without having it follow an actual cycloid; otherwise friction would have quickly stopped the oscillations.

From a more abstract viewpoint, the problem is how to draw a cycloid without using an outline to guide the pencil.

Huygens' idea was to constrain the motion of the pendulum's string using specially shaped profiles. This way the weight, which if oscillating freely would describe an arc of circumference, would move on a different curve when constrained by a profile that is called its involute. It is possible to draw the involute of a curve - here we see the involute of a circumference - by coiling or uncoiling around the given profile a thread at whose end is a pencil. At the same time, if we place any object at the end of the thread, this will move along the involute of the curve, even if this latter curve is not physically present.

When we trace the involute of the circumference, we already know the profile, which is the circumference, and we want to find its involute. In the case of the pendulum, we are faced with the inverse problem. We want

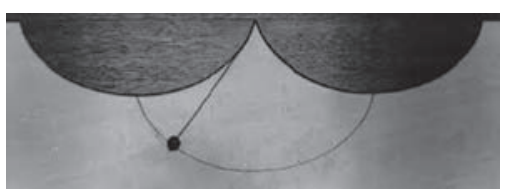

the weight of the pendulum described on a cycloid; therefore, we must find a profile whose involute is a cy-

cloid. Huygens showed that this happens when the profile is also a cycloid identical to the one sought.

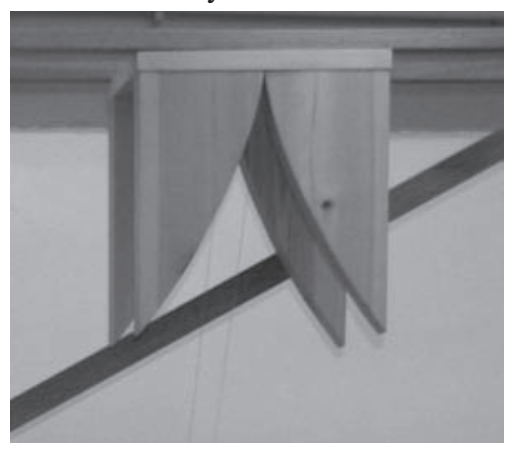

Thanks to this result, by swinging the pendulum between two wings shaped like cycloid arcs, the weight will move along a cycloid and its oscillations will be isochronous.

This becomes immediately a museum sequence. Successive installations show the generation of the cycloid, its isochronal properties, the notion of an involute and the tracing of the involute of a circumference and of a cycloid, and finally a cycloidal pendulum whose isochronous oscillations are compared with the non-isochronous oscillations of a traditional pendulum.

None of these steps would show a mathematical object at work by itself. When seen together, they allow it to emerge from the physical objects on display, showing the role of mathematics as a regulator of the functions of real objects.

The cycloidal pendulum and Archimedes' spiral also illustrate another aspect of mathematics that is relevant to a museum: history. In the public's imagination, mathematics is often seen as immanent. There are no revolutions or paradigm shifts in mathematics: once proved a theorem is proved forever. In fact, although traces of Euclid's and Archimedes's results can be found in the most recent mathematics, the condition for this is that they be subject to constant transformation and drastic reappraisals. Just as a barrel which is refilled with new wine every year preserves traces of ancient vintages, classical mathematics is part of modern mathematics thanks to constant decanting and blending. If it's true that the history of mathematics does not contain any sudden changes in direction, it is not exempt from transformations and changes: remembering past theories often restores depth to a science that is too often focused only on the present.

But let's go back to the museum and in particular to the proofs we started with. Although, as we said, they contain mathematics that is alive and kicking, this does not mean that a visitor's interest and attention can be attracted just by showing a proof - even of an important and well known theorem like Pythagoras' theorem.

At the Giardino di Archimede (the Garden of Archimedes), the proof of Pythagoras' theorem is associated with puzzles through which, with the same pieces, one can build either the square of the hypotenuse or the square of the legs and thus verify the validity of the result. Conceptually, the puzzles are not better than the "hydraulic" demonstration: in both circumstances it is a matter of geometry of the eye and of the particular case. However this does not pretend to be a proof; it just recalls the content of the theorem and helps visitors to remember it through play. The puzzles, moreover, can be read on more than one level: firstly, as a game and an intellectual challenge, as they are much less easy than they look at the start; but also as the vector for a less superficial mathematical concept. In this case, the underlying result is that two polygons are equivalent (that is, have the same area) if and only if they are equidecomposable (that is, are made from the same pieces). The proof of this result goes beyond popularisation within the confines of the museum but can be discussed at a later stage, for example in school; here one can limit oneself to the remark that the same result does not hold in three dimensions.

Subsequently, other shapes like hexagons or stars are used in place of squares, which shows that Pythagoras' theorem is also true for these polygons and, in general, for any similar figures. At a higher level, this is explained with the fact that the areas of similar figures constructed on the legs and the hypotenuse of a right triangle are proportional to the squares of these segments. Finally, by applying this result to semicircles we come to the squaring of the lunulae.

In this way the proof of Pythagoras' theorem is no more an end to itself but it is part of an itinerary which starts with the classical enunciation of the theorem and passes through the equidecomposability and variation of the area by similarity to arrive at the issue of the quadrature of lunulae. All this is presented as games which can stimulate attention and learning at various levels.

We could continue with more examples but I think that these ones illustrate quite clearly one of the main points of the idea of museum that we have at the Giardino di Archimede. Abstract thinking, and mathemat- 
ics in particular, emerges from the physical reality of the objects on show not just by pushing buttons and experimenting isolated phenomena, each of which speaks a different language, but by organising objects and principles onto a meaningful path. I think this is the only way to involve the visitor in the world of mathematical creativity: to make mathematics, instead of just evoking it.

But isn't it the same for physics, astronomy, chemistry - for any other science? Science, and consequently its popularisation, is not just a sequence of experiments, each with its nice ad hoc explanation; it consists of organising a mass of phenomena into a theoretical structure. More than showing objects, one has to tell stories. Or rather, scientific objects and experiments must be used to tell stories about science. A good museum for mathematics is a place which tells good mathematical stories.

There aren't recipes that guarantee that the stories will be good ones. But there are some tricks that help us avoid serious mistakes.

First of all, we must always remember that communication involves two agents: the communicator and the listener. Even if the latter is not always able to comment or respond, they can always express their dissent simply by interrupting communication. The visitor's attention is not guaranteed and must be earned every time. In order for this to happen, the level of communication cannot be too difficult or visitors will leave because they don't understand. And it cannot be too easy or listeners will be bored and distracted. Most of all, their attention must be captured by showing them something that is not extraneous to them and that can be linked to a familiar situation or to a nucleus of acquired knowledge, even outside the world of mathematics.

At the Giardino di Archimede, we moved along three lines:

1. History. Although all our exhibitions contain a certain amount of history, some of them are more fundamentally historical. For example, $A$ bridge across the Mediterranean is about medieval Arabic mathematics and how it was brought to the West by Leonardo Fibonacci, while Mathematics in Italy tells the history of Italian mathematics and mathematicians from Napoleonic times to the Second World War. In both cases, the horizon is not limited solely to mathematics; it is seen as part of the political, economic and social his- tory, so that visitors can integrate it into their general historical knowledge, in which the history of science, and mathematics in particular, often holds a marginal position.

2. Play. Thanks to its universal character, play can be one of the most important ways of transmitting knowledge and many of the exhibitions in the Giardino di Archimede stimulate the interest of the visitor through games: puzzles in Pythagoras and his theorem; a swing in Helping Nature; Hanoi towers or Hex in Weapons of Mass Instruction. It is however necessary to maintain the balance between two opposite dangers: that the game remains an end to itself, without communicating its mathematical content, or that the latter be preponderant and make the game interesting only for the initiated.

3. Daily life. Often mathematics is seen as being only of interest to specialists, with no relationship with everyday life. Exhibitions like Beyond the compass and Helping nature show objects and mechanisms with which we interact every day and how their creation and their operation is based on often hidden mathematical properties; this always surprises and engages visitors.

These three aspects can intersect and combine in various ways to create a mathematical story. Sometimes only one of them is present, as in the historical exhibitions, but in many cases history is intertwined with play or with everyday life. When all three are present, we can say that visitors are really able to touch the abstract in their relationship with mathematics.

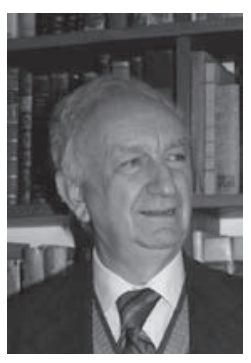

Enrico Giusti [giusti@math.unifi.it]was a professor of mathematics at the University of L'Aquila and later at the Universities of Trento, Pisa and Florence. He retired from teaching in 2010. His scientific interests include partial differential equations, the calculus of variations and differential geometry; later he focused on the history of mathematics and more recently on the popularisation of mathematics. In 1999 he founded "Il Giardino di Archimede", the first museum ever devoted entirely to mathematics. 


\section{Unity and Disunity in Mathematics}

Bernhelm Booß-Bavnbek (Roskilde University, Denmark) and Philip J. Davis (Providence, RI, USA)

\section{Foreword}

While dictionaries have produced a number of definitions of the word "unity", the sense in which we employ the terms unity or disunity will emerge from what follows. The unity of mathematics has been proclaimed and celebrated by numerous mathematicians of the first rank, most recently by M. F. Atiyah and I. M. Gelfand. Atiyah has found the notion of unity in the interaction between geometry and physics while Gelfand asserts that its "beauty, simplicity [!], exactness, and crazy ideas" as well as its capability of abstraction and generalisation are all hallmarks of an inner unity.

In other places, we have elaborated this by pointing out that the unity of mathematics has, as a consequence, its power to compactify experiences in a form capable of being transferred and modified or adapted to new mathematical situations. Existing knowledge is mustered towards creating the iterative sequence description, prescription/design, execution, comparison.

An additional claim of unity is that mathematics research for at least the last 200 years has been largely dominated by a single methodology: that of rigorous proof. These assertions add up to the "Unity of Mathematics", a well-known and well publicised, predominantly philosophical claim that has been promoted in international conferences on the topic.

Important declarations can be found in the volume The Unity of Mathematics edited by Etingof et al. [7]. In this book about 20 articles on a variety of topics, written by preeminent authorities, have been anthologised and advanced under the slogan of unity. What a casual reader may wonder about this collection is whether the various authors are capable of or are interested in understanding what their fellow authors have written. The second named author, a professional who has functioned for some years as a researcher, an applied mathematician and an educator, found all the articles incomprehensible. He rejects the argument that since "mathematics is a universal language", with a bit of study he would find them all understandable. This would be similar to the assertion that with a bit of goodwill in the universe, the lion will lie down with the lamb and universal peace will reign on earth. Thus, while the editors trumpet the unity of mathematics, the contents of the book assert equally well its disunity. This is an instance of a simultaneous and dual nature, expressed by unity/disunity, that permeates the field.

Grattan-Guinness ([8], p. 490) points out that by the turn of the 20th century, "a snobbish preference for pure mathematics over applications became more marked ... so that the profession tended to separate into two components. In Berlin and elsewhere in Germany, purism became an explicit creed for the professional..."

We have had (and still have) the Journal für die Reine und Angewandte Mathematik (Crelle's Journal, founded
1826) ${ }^{1}$ and the Journal de Mathématiques Pures et Appliquées (Liouville's Journal, founded 1836). Thus, while the existence of what is pure and what is applied would seem to proclaim disunity, they often stand united.

Yet, we believe that the phrase "unity of mathematics" expresses a dream, an ideal that doesn't exist. We shall point to diachronic and cross cultural disunities, to semantic, semiotic and philosophic ambiguities and to the non-acceptance of certain mathematical texts by some practitioners of the subject. But to deny the strivings towards unity would be misleading and not constructive.

\section{Extent and practices of the mathematical enterprise}

The richness of the mathematical enterprise can be exhibited in many different ways and can be used to show both its inherent unity and its disunity. The number of mathematicians currently in the world has been estimated at 100,000 . The number of mathematics books in the Brown University Science Library is approximately 32,000. Many of these books carry a title that implies the gathering together and the wedding of information around a single topic. Within this "marriage" unity may be found, e.g. Analytic Function Theory, Circulant Matrices and Elliptic Boundary Problems for Dirac Operators.

Such books display the mathematical enterprise at a high level of theory and application. At the informal level of discourse and practice, a life today without the continuous use of mathematics in commerce, finance, science and technology, medical practice, art, sports, etc. is inconceivable. We are living in a highly mathematised social and technological world.

Let us look at the published material. The subject classification scheme of the Mathematical Reviews and Zentralblatt MATH (MSC 2010) at the macro level lists almost a hundred mathematical subjects, random examples being: 00 General; 13 Commutative Rings and Algebras; 42 Fourier Analysis; 83 Relativity and Gravitational Theory; and 97 Mathematical Education. At the micro level, within each group there are subgroups such as 13A15: Ideals and Multiplicative Ideal Theory.

Since mathematics has the aspect of a language, it is instructive to compare it with the classification schemes for literature and rhetoric. The Dewey Decimal Classification System (1878 and with constant updates) at the macro level lists 100 categories numbered from 800 to 899. Since mathematics also has the aspect of an art, it would be of interest to compare its classification schemes with the MSC 2010.

\footnotetext{
1 In an old and sceptical play on words, Reine und Angewandte Mathematik become Reine undangewandte Mathematik (pure inapplicable mathematics). Notice also the predominant plural in the French use of the term mathématique.
} 
To some extent, each macro and micro mathematical subject has its own techniques and its own intellectual resources and devotees who both utilise and enrich its resources. While there may, indeed, be some connections between e.g. potential theory and non-associative algebras, elaborate abstract graphs and tree structures have been drawn, linking or uniting selected mathematical topics. This can be done even with an individual theorem. The second named author recalls that as a graduate student in Professor Hassler Whitney's course on topology, he made a tree structure for one of the major theorems in topological group theory showing how many significant theorems fed into the final statement as sub-results.

The frequent cross-collaboration between experts indicates a certain degree of unity and coherence in the field of mathematics. A newly discovered and surprising application of Theory A to Theory B is not a rare occurrence and is often put forward as the strongest evidence of the unity of mathematics.

On the other hand, professional specialism is rife and there is an often unconscious snobbism that downgrades interests other than one's own. "Life is too short", one says, "for me to care about everything let alone to know everything." A consequence is that one professional may have precious little to chat about with his office neighbour. The fact that Brown University has two Departments of Mathematics, one pure and one applied, with an historic record of intra-professional isolation is significant evidence of disunity.

\section{Diachronic and cross cultural disunity}

Written mathematics is easily 4000 years old. It has been created by people and has served people for a variety of purposes. A mathematician lives in a sub-culture at a certain time and place. A piece of mathematics does not exist only in a sequence of special symbols because the naked symbols are essentially uninterpretable. The symbols are embedded in a cloud of knowledge, meanings, associations, experiences and imaginations that derive from the particularities of time, place, person and the enveloping society.

Pythagoras asserted that 3 is the first male number. In certain Christian theologies it is the number of the Godhead. If in Chaldean numerology the numbers 1, 10, 19 and 28 are "ruled by the sun", the meaning of and the belief in those words may easily escape our readers. 666 is the "Number of the Beast" and there are numerous other mystic and arithmetic relationships. One might now say that such statements as " 3 is the first male number" are not mathematics, pure or applied, but such a judgment delimits the notion of mathematics to only what is acceptable to today's establishment.

More generally, there are mathematical narratives that attempt to interpret very old texts. Methodologies of interpretation go by the fancy name hermeneutics and a frequently utilised interpretive device goes by the name present-centred history. This is where the past is described by an interpreter who uses the full knowledge of developments of subsequent importance. But can the interpreter do better than that and really get into the minds of the past creators? Only imperfectly. Eleanor Robson, noted for her studies of ancient Babylonian mathematics, suggests as much. Nonetheless, efforts have been made in this direction and Robson refers in [12] to the work of Henk Bos and Herbert Mehrtens who considered the relation between mathematics and the enveloping society and to the work of David Bloor for whom mathematics was a social construction toute courte.

\section{Semantic ambiguity}

We may write down the sequence of symbols $x$ 느 $\cap \sigma \sum \equiv 6$ and claim that this is a piece of mathematics. But this claim cannot be substantiated on the basis of the symbols alone. To provide meaning, every mathematical statement must be embedded in a sentence written in some natural language (English, German, etc.). Furthermore, significance of these symbols as mathematics cannot be established if its knowledge were limited as a private revelation to one and only one person.

Here is an example of private jottings. Paul Valéry (1871-1945) was a distinguished French poet and litterateur. He was also a bit of a "math buff".

Here is a clip from a page of his Cahiers (Notebooks).

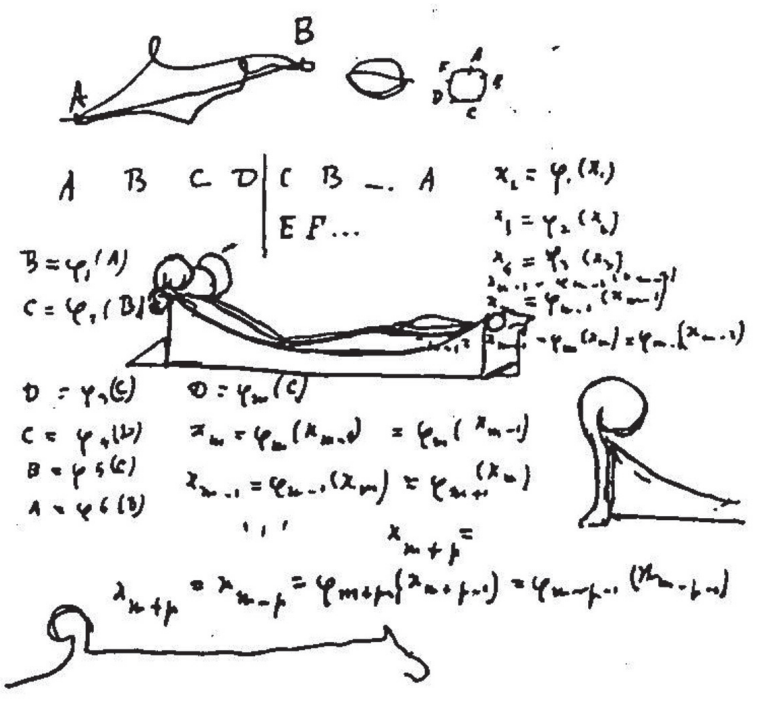

Jottings extracted from Paul Valéry, Cahiers II (1900-1902), Paris 1957, p. 608. Copyright by Centre National de la Recherche Scientifique.

What is going on here? For a discussion of the attempt to interpret these jottings, see P.J.D.: Bridging Two Cultures: Paul Valéry. ${ }^{2}$

Semiotic ambiguity and the problem of synonymy Can it be determined when two mathematical statements, phrased differently, are asserting the same thing? "The awkwardness of equality" has been discussed by Barry Mazur in his article When is one thing equal to some other thing $?^{3}$

\footnotetext{
2 Svenska Matematikersamfundet Medlemsutskicket, 15 May 2009. Reprinted in: The Best Writing on Mathematics. Mircea Pitici, 2010.

3 Pp. 221-241 of [9].
} 


\section{Scepticism, doubts, non-acceptance}

While each of the sections of this article could be elaborated into a book, the doubts, dilemmas and angst surrounding certain mathematical concepts could be shaped into a mathematical comedy. They have been treated in extenso by a variety of authors. ${ }^{4}$ We shall recall briefly and simplistically and not in historical order a number of the objections that have been raised.

- Zero: How can zero be a number? Common sense asks: how can nothing be treated as something?

- One: A number must express numerosity or multiplicity.

- Fractions: How can $1 / 2=2 / 4$ when half a pie is not the same as two pieces of pie that have been cut into quarters?

- Negative numbers: How can less than nothing be something? Scepticism about the interpretation of negatives lasted far into the 19th century. António José Teixeira, mathematics professor in Coimbra, wrote in 1890 that he did not like the proportion $1:-1::-1: 1$ and asserted that "the negative quantities do not possess any arithmetical existence".

- Irrational numbers: $\sqrt{2}$ derives from the diagonal of the unit square. It is a line segment whose length exists and yet has no existence as a number. (Note the legend of Pythagoras offering up a hecatomb of oxen upon this discovery.)

- Imaginary numbers: $\sqrt{-1}$, etc. "Imaginary numbers are a fine and wonderful refuge of the divine spirit almost an amphibian between being and non-being" - Leibniz.

- Infinity: How can there be an infinity when the concept is germinated, represented or defined and operated on by a finite number of symbols? Infinity, so some claim, is a property only of the Godhead.

- Infinitesimals: These are "the ghosts of departed quantities" according to the philosopher George Berkeley.

The ideas surrounding number, particularly the idea of infinity, have been fertile fields for philosophers, theologians, neo-Platonists, numerologists, mystics and even cranks to plough. Indeed, in the early days of the development of the subject, mathematicians could hardly be distinguished from these other "occupations".

Moving briefly to mathematical functions (i.e. curves, graphs), we come across another objection:

- Dirac Function: How can a function that is zero on $(-\infty,+\infty)$ except at one point have a positive area "underneath" it?

Over the years - sometimes it took centuries - the concepts just mentioned and considered problematic have been totally absorbed into mainstream mathematics by, among other things, having been embedded within axiomatic, deductive formalisations of a traditional type. Moreover, and this is of great importance, these concepts have proved useful to science, technology and even to mathematics itself, as well as to a wide variety of humanistic concerns.

Thus, it would seem that both within and without mathematics, utility confers ontological reality and justi- ficatory legitimacy. An increase in utility is accompanied by additional legitimacy and an abatement of scepticism. Yet the concept of utility and the ideas of "more" utility and "less" utility are admittedly vague and have been questioned. Utility to what and to whom? The late Richard Hamming, a very down-to-earth and practical type (he has been dubbed a techno-realist ${ }^{5}$ ), referring to one standard and basic concept of what is now termed "real analysis", wrote:

"Does anyone believe that the difference between the Lebesgue and Riemann integrals can have physical significance, and that whether say, an airplane would or would not fly could depend on this difference? If such were claimed, I should not care to fly in that plane."

Profitable utility as a pre-condition for legitimacy stands in low regard in certain portions of the mathematical community. Wasn't it Euclid who is quoted as saying "Give the student a coin for he demands to profit from what he learns"? Other criteria besides utility exist for justification, legitimacy and acceptance: for example, the process of mathematical proof or physical verification. There are other instances of non-deductive approaches and all of these add up to what might be called modes of theorematic evidence.

Zermelo did not believe Gödel's proof. The scepticism of Kronecker, Poincaré, Zermelo, E. Picard, Brouwer, Hermann Weyl, Wittgenstein, Errett Bishopp, etc. regarding the concepts of Cantor has been well documented. Another quote from Richard Hamming sums this up: "I know that the great Hilbert said 'We shall not be
driven out of the paradise that Cantor has created for
us', and I reply I see no reason for walking in."

In the opposite direction, the great G. H. Hardy asserted he was interested in mathematics only as a creative art and that none of his work in it was of any utility.

\section{Philosophic ambiguity}

Originally there was one formal geometry: that of Euclid. After Bolyai and Lobatchevski, there were three and after Riemann, an infinity of geometries. Prior to the end of the 19th century, there was one philosophy of mathematics: that of Platonism. Now there are easily a number of distinguishable philosophies and together with these there are variations that exhibit the Freudian "narcissism of slight differences". There are platonism, logicism, formalism, intuitionism (constructivism), empiricism, conventionalism and culturalism/experientialism. In probability

\footnotetext{
${ }^{4}$ E.g. Brian Rothman, Signifying Nothing: The Semiotics of Zero, or Gert Schubring, Conflicts Between Generalization, Rigor, and Intuition: Number Concepts Underlying the Development of Analysis in 17-19th Century France and Germany. See also: P.J. Davis: Mathematics and Common Sense, Chap. 12.

5 Richard Hamming: An Outspoken Techno-Realist. SIAM NEWS, 16 Nov 1998.
} 
theory there are the frequentists vs. the interpretations of likelihood. We call ourselves "mild social constructivists". Others have called us simply "mavericks".

Despite the often cited saying of Pythagoras that "All is Number", we doubt whether the Pythagoreans would have called the Songs of the Sirens mathematics. There is a separate and unique corpus of material, created over the centuries by the human intellect, that has been gathered together and has been called mathematics. Unity vs. disunitry? E pluribus unum.

\section{Acknowledgements}

We wish to acknowledge fruitful discussions with Sussi Booß-Bavnbek, Ernest S. Davis and Katalin Munkacsy.

\section{Bibliography}

[1] Marcia Ascher, Ethnomathematics: A Multicultural View of Mathematical Ideas, Pacific Grove, Calif. 1991.

[2] Paul Benacerraf \& Hilary Putnam, eds., Philosophy of Mathematics: Selected Readings, Cambridge Univ. Press, 2nd. Ed., 1983.

[3] David Berlinski, Infinite Ascent: A Short History of Mathematics, Modern Library, 2005.

[4] Ubiratan D'Ambrosio. Ethnomathematics and its Place in the History and Pedagogy of Mathematics, For the Learning of Mathematics 5/1 (1985), 44-48.

[5] Philip J. Davis, Mathematics as Narrative, SIAM News 45/5 (June 2012).

[6] Heinz-Dieter Ebbinghaus, Ernst Zermelo, Springer, 2007.

[7] P. Etingof, V. Retakh, I.M. Singer, eds., The Unity of Mathematics:
In Honor of the Ninetieth Birthday of I.M. Gelfand, Birkhäuser, Boston, 2006.

[8] Ivor Grattan-Guinness, The Norton History of the Mathematical Sciences, W.W. Norton, 1997.

[9] Bonnie Gold \& Roger A. Simons, eds., Proof \& Other Dilemmas: Mathematics and Philosophy, Math. Assoc. America, 2008.

[10] Loren Graham and Jean-Michel Kantor, Religious Heresy and Mathematical Creativity in Russia, Mathematical Intelligencer 29/4 (Nov 2007), 17-22.

[11] Hal Hellman, Great Feuds in Mathematics, John Wiley, 2006.

[12] Eleanor Robson, Mathematics in Ancient Iraq: A Social History, Princeton Univ. Press, 2008.

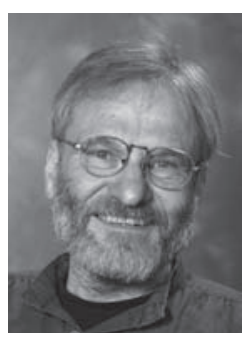

Bernhelm Booß-Bavnbek[booss@ruc. $d k J$ is a senior lecturer of global analysis and mathematical modelling at Roskilde University (Denmark). His newest book "Index Theory with Applications to Mathematics and Physics" is in print.

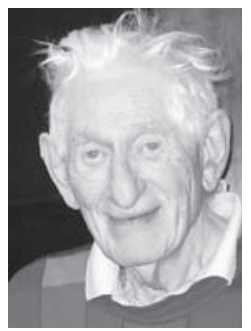

Philip J. Davis [philip_davis@brown. edu] is a professor emeritus of applied mathematics at Brown University and is an independent writer, scholar and lecturer. He lives in Providence, Rhode Island, USA.

\section{Episciences: a publishing platform for Open Archive Overlay Journals}

Jean-Pierre Demailly (Université Joseph Fourier, Saint-Martin d'Hères, France) on behalf of the epi-math committee

While ever-increasing prices of scholarly publications have raised concerns for a long time, a growing number of interesting evolutions have taken place in the scientific community, especially in mathematics: a universally accepted document format (TeX/LaTeX), a widely adopted electronic open archive with worldwide coverage (arXiv) and more recent attempts to create open access journals and open discussion forums. In the context of commercial publishers seemingly unlikely to propose affordable and sufficiently open solutions for scientific publications, the mathematical community seems ready to adopt new publishing models and get actively involved in the related developments. Since creating open access electronic platforms has become technically easy, the obstacles are more historical and sociological than anything else. What is needed is a convenient framework that gives our community the tools to assess, correct, certify, archive and make widely available their production.

"Episciences" is a project hosted by the CCSD (Centre pour la Communication Scientifique Directe, located at Lyon University and attached to CNRS/IN2P3, a network of nuclear physics laboratories), in collaboration with Institut Fourier (a mathematics research department at Grenoble University, France). The CCSD develops the open archive "HAL" and also maintains a complete mirror of arXiv and an interface between HAL and arXiv. The aim of "Episciences" is to provide a publishing platform that makes it as easy as possible to host, run or create open archive overlay journals (hopefully a large number of them, independent of each other). Technically, the platform will rely on the HAL archive.

An overlay journal is a scientific journal that is focused on the peer-review process, and backed by one or several open archives for its diffusion and data handling. The first goal is to make it possible to efficiently run a journal at minimal cost and the second goal is to enforce a unified open access to the electronic version. In recent years, some print journals briefly became overlay journals but this experiment could not be sustained over an extended period. Episciences is an attempt at electronic- 
only overlay journals, providing a priori a much reduced operational cost.

The journals hosted in Episciences, called epijournals, will be either new publications or existing journals that have accepted to collaborate. Episciences will start with a focus on mathematics but might extend to other fields. The platform is expected to start its operation during the first semester of 2013.

The editorial process envisioned is quite standard: authors submit their articles after making them available in the arXiv or in HAL and provide the ID of their e-print to a specified epijournal of their choice. The editorial board of that epijournal handles the submission exactly as for a traditional scientific journal, appointing referees and deciding to publish - or not - when the report is received. If the article is accepted after suitable corrections have been made, it is subsequently listed on the webpage of the journal as a link to the actual file, the final version of which is stored solely in the open archive.

Episciences will provide all relevant services to help the epijournal editorial boards in managing the various tasks required by the editing and refereeing process. In particular, it will provide a global web platform devoted to implementing these services. One feature that is expected to save editors a lot of time is an email channel that will enable, between the submission and the final decision, the authors and the referees to communicate together while keeping the referee anonymous. The referee will therefore be able to suggest/ask for all needed corrections and modifications from the author and generally the editor will not need to act as an intermediary before a final report is written. The most interesting feature, though, is that the CCSD will entirely develop and host the platform itself, so that extra technical costs are reduced to zero, allowing epijournals to operate in an open access model without having to require author fees or reader subscriptions. A unified presentation of the articles will normally no longer be needed. Instead, Episciences will offer a normalised (La)TeX format suitable for A4/letter printing of the articles; editorial boards and referees will have to take care that the articles are typeset in a professional manner. In addition to giving their scientific views on research papers, the epijournal editorial boards will have the opportunity to post reviews concerning the accepted papers - Episciences encourages this option but does not want to make it compulsory. These reviews would be meant to stress in a positive manner the contributions made by authors: their scopes and perspectives, their relations with existing literature, etc.; they could be based in part on material sent by referees (in an anonymous way and with their consent). Beyond editorial reviewing by epijournals, some other types of reviewing activity, similar to the Math Reviews, for example, might also take place on the Episciences platform, although this possibility is still under investigation at this point.

The Episciences initiative will be supervised in each discipline by a dedicated "Epicommittee". Such an "epimath" committee ${ }^{1}$ was created in January 2013. Its role is to stimulate the constitution of editorial boards willing to create new epijournals, especially thematic epijournals in areas not yet covered, to manage possible takeovers of existing journals willing to join, and finally to treat any related ethical and professional issues. Members of the Epicommittee may or may not take responsibility of an epijournal. Reaching a wide geographic coverage of the various mathematical communities worldwide is highly desirable in the short-term.

In political terms, let us conclude this discussion by stating that the Episciences project supported by CCSD aims to develop a viable alternative to existing economic models currently in use in scientific publication. The main goals are:

- Enforcing open access to electronic versions of research papers, while assessing and validating them.

- Allowing the efficient management of newly created electronic journals by reducing their operational costs.

- Developing the infrastructure for modern indexing and searching tools in the context of scientific data management in the longer term.

We hope that mathematicians will consider Episciences as an opportunity to move scientific publication in directions that are better suited to the needs of the scientific community and that experts in the various domains of mathematics will help to constitute many editorial boards, so as to manage many high standard epijournals. Collaboration with the open access archives such as HAL and arXiv is instrumental in this process and, in any case, we encourage scientists to take care that the final versions of their papers - including corrections arising from referees and reviewers - are returned to the open archives. All existing mathematical journals that promote high standards and have a compatible (open) publishing policy are encouraged to join Episciences and use its facilities.

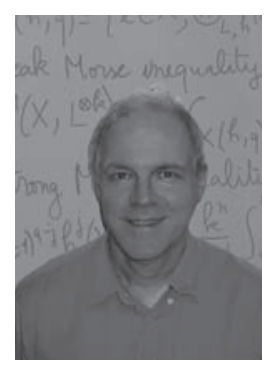

Jean-Pierre Demailly [Jean-Pierre.Demailly@uif-grenoble.fr] has been a professor at the Universite Joseph Fourier, Grenoble, since 1983, and a member of the Institut Universitaire de France since 2002 and the Académie des Sciences $^{2}$ since 2007. He is a specialist in complex analytic geometry.

1 The current members of epi-math (as of 25 January 2013) are: Sun-Yung Alice Chang, Ingrid Daubechies, James Davenport, Jean-Pierre Demailly (one of the initiators of the project along with Benoit Kloeckner and Ariane Rolland at Institut Fourier), Timothy Gowers, Greg Kuperberg, Gadadhar Misra, Junjiro Noguchi, Peter Olver, Thomas Peternell, Terence Tao, Wendelin Werner, Shing-Tung Yau and Xiangyu Zhou. Peter Olver is also Chair of the CEIC (Committee on Electronic Information and Communication) at the International Mathematical Union and James Davenport is another member of the CEIC.

2 The Académie des Sciences has just initiated a Committee for Scientific Edition. Its role is to investigate issues related to the evolution of technology and related practices, especially open archives and electronic publications. The Académie intends to publish a report and recommendations in the course of 2013. 


\section{On the "Call for Balanced Negotiations with Scientific Publishers"}

Benoît R. Kloeckner (Université Joseph Fourier, Saint-Martin d'Hères, France)

Near the end of 2011, as a scientific advisor for the mathematical library of the Institut Fourier (Grenoble, France), I had to choose whether we should unsubscribe from some journals published by Springer. The goal was to reduce our spending with this publisher at a time when institutional partners were conducting national negotiations with Springer to renew the contracts of French research institutions.

There were tight constraints, since a majority of our Springer subscriptions where "pledged": the contract that was ending prevented us from unsubscribing from these titles unless we took new subscriptions for at least as large a cost. After trying to cut out unpledged titles, I realised that we could considerably reduce our spending, without cutting out too important journals, if we could unsubscribe freely. Moreover, without this possibility we would be very dependant on the budget we get from our university specifically for the library (which might dry up any year); if this funding were lost and the new contract with Springer was similar to the preceding one, we would be forced to unsubscribe almost all non-Springer titles. Since the little information we got from the negotiations was not very encouraging, together with the chief librarian Francesca Leinardi and the laboratory director Gérard Besson we decided to take action.

First, we launched a discussion on this issue on email lists gathering mathematicians playing roles in departments or libraries in France. It was easy to see that almost everyone agreed about the problem but that something was needed to clearly express this consensus. We then decided, with the support of the council of the Institut Fourier, to launch a petition entitled "L'Appel pour des négociations équilibrées avec les éditeurs de revues scientifiques" ("Call for balanced negotiations with scientific publishers"), which can be read at http://www-fourier.ujf-grenoble.fr/petitions/ index.php?petition $=3$.

This call targeted Springer, mostly because of the ongoing negotiations; it was addressed to the institutional negotiators (CNRS, INRIA, Couperin) to ask them not to accept any term that would prevent us from unsubscribing freely or that would impose large annual price increases. The signers asserted that they agreed not to have wide access to Springer journals if it was necessary to avoid such terms; this was designed to give the negotiators the option of walking out of the negotiations.
To cut a long story short, the call was widely disseminated among the French mathematical community (we did not have the means to disseminate it much more widely) and signed by a majority of mathematical research units and by many mathematicians, as well as by some computer science, physics and economics departments, but it had little effect on negotiations. We still have as many pledged journals as before, from which we cannot unsubscribe for three more years, and it seems difficult to hope for a significant improvement for the next contract to be signed in 2014.

However, I do not think the call was a failure: we now know that we, as a community, want to fix the publishing system. And a lot has been tried already, as has been witnessed by high energy physics (see the very interesting SCOAP3 consortium), by biology and health science (with PLoS, the leading open-access publisher relying on article processing charges rather than subscription) and mathematics (with the launch this Autumn of the Forum of Mathematics by Cambridge University Press - see also http://publishing. mathforge.org/ for discussions on the future of mathematical publishing).

Each of us could be unconvinced by some of these developments but none can continue to blame commercial publishers while pretending we cannot do anything. There are many ways to act, if only by the choice of journals where we submit our work. It is up to us to shape our publishing system for the next decades and it is happening now.

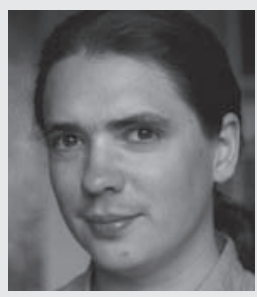

Benoît R. Kloeckner is an associate professor at the Institut Fourier (Université Grenoble 1, France, and CNRS) and scientific adviser of its library. 


\section{The British Society for the History of Mathematics}

Robin Wilson and Raymond Flood (Oxford, UK)

The British Society for the History of Mathematics (BSHM) was founded in 1971 and currently has about 300 members from around the world. Its aims are:

- to promote and encourage research into the history of mathematics and to disseminate the results of such research.

- to promote and develop for the public benefit, awareness, knowledge, study and teaching of the history of mathematics.

- to promote the use of the history of mathematics at all levels in mathematics education in order to enhance the teaching of mathematics for the public benefit.

\section{The first meetings}

The circumstances of the Society's founding were described by its first Secretary and later President, Dr John Dubbey (subsequently Vice-Chancellor of the University of Malawi) [1]:

The idea of a Society for the History of Mathematics was initiated by the late Arthur Morley (the Society's first treasurer). He had read my book The Development of modern mathematics (Butterworth 1970) and suggested we meet to consider the formation of a society.

They duly met in the buffet at St Pancras Station, London, and agreed to convene an open meeting on the history of mathematics to see how much interest there would be. Dubbey continues:

We approached Gerald Whitrow [of Imperial College, London], who gave his enthusiastic support and agreed to chair this exploratory meeting. This took place at Thames Polytechnic [now the University of Greenwich] on 2 July 1971 with, to the surprise and delight of the organisers, over fifty participants.

The purpose of the exploratory meeting was to discuss:

- the existing situation in the history of mathematics, in terms of research, literature, teaching, availability of information, etc.;

- the value of studying the history of mathematics at universities, polytechnics and colleges of education;

- the formation of a national society concerned with developing the history of mathematics;

and the meeting began with five lectures:

John Dubbey (Thames Polytechnic) on The history of mathematics in universities and polytechnics;

Arthur Morley (Nottingham College of Education) on The history of mathematics in colleges of education;
Leo Rogers (Digby Stuart College) on Relations between the history and pedagogy of mathematics;

J. V. Pepper (North-East London Polytechnic) on Thomas Harriot (1560-1621);

Ivor Grattan-Guinness (Enfield College) on Literature and methodology in the history of mathematics.

The ensuing discussion underlined the participants' desire for a society of this nature, and an interim committee was elected to draw up rules for the Society and convene a 'foundation conference' in about six months' time.

In spite of a couple of critical letters to the interim committee attacking the history of mathematics as a credible academic discipline, this opening meeting duly took place at Thames Polytechnic on 17 December, with an audience of about forty people. A formal lecture on Geometry from Riemann to Whitehead was presented by Prof. George Temple FRS, Emeritus Professor of Oxford University and a former President of the London Mathematical Society. To run the Society a committee was elected, consisting of the members of the interim group (Dr Gerald Whitrow as President, Dr John Dubbey as Secretary, and Mr Arthur Morley as Treasurer), together with Dr Margaret Baron (Stockwell College, London) and Dr J. D. North (University of Oxford). The annual subscription was set at $£ 1$.

During the next year two meetings were held - on 20 May at the Nottingham College of Education, with speakers Henk Bos (Utrecht) on Differentials and higher differentials from Leibniz to Euler, John Dubbey on Charles Babbage, mathematician and computer pioneer, Ivor Grattan-Guinness on Contiguous functions, and Stuart Hollingdale on The history of numerical analysis, and on 15 December at the Polytechnic of the South Bank (London), with the Annual General Meeting and two guest speakers: George Molland on Modes of description of geometrical figures: Descartes and antiquity and D. T. Whiteside on Astronomical eggs, laid and unlaid, in Keplerian planetary theories. By this time the membership had reached 142 .

\section{Presidents of the BSHM}

1971 Gerald Whitrow

1995 Steve Russ

1974 Clive Kilmister

1977 John Dubbey

1980 Graham Flegg

1997 J. V. Field

1983 Frank Smithies

2000 Peter Neumann

2003 June Barrow-Green

1986 Ivor Grattan-Guinness

1989 Eric Aiton

2006 Raymond Flood

1992 John Fauvel 


\section{The Society's activities}

The ensuing years experienced a great increase in both the number and scope of the BSHM's activities.

\section{Residential meetings}

The first weekend meeting of the Society took place in September 1973 at St Hugh's College, Oxford University. This gathering was so successful that residential meetings soon became a regular feature in the Society's programme. In the 1970s residential meetings took place at a college in Birmingham and at the Universities of York, Nottingham, and Cambridge. At first each meeting covered a diverse range of topics, but in 1979 the BSHM returned to Oxford (Pembroke College) for its first themed weekend, on Geometry and physical theory, 1630-1930.

These single-topic weekends continued into the 1980s with residential meetings on the History of computing and algebra, Mathematics education, Renaissance mathematics, History of probability and statistics, and From Fourier to fractals, and have been held, usually once a year, ever since. In 2012 there were two residential meetings, both in conjunction with the Oxford University's Department for Continuing Education - Turing's worlds, celebrating the centenary of Alan Turing's birth, and Mathematicians and their gods, on historical connections between mathematics and religion - and in June 2013 the featured topic is Geometry: from Euclid to Einstein.

\section{Regular meetings}

As can be seen from the previous paragraph, there was an increasing variety in the topics covered in the Society's activities. Among the many one-day meetings in the 1970s and 1980s were gatherings on Babylonian and Egyptian mathematics, Calculating engines, Women mathematicians, The history of logic, Three founders of electromagnetism (Ampere, Faraday and Maxwell), and Music, perspective and mathematics. The early London meetings took place in places ranging from the British Museum, Science Museum and the Institute of Physics, while outside the capital our meetings were held at the Universities of Oxford, Cambridge, Birmingham, Warwick, Keele and Newcastle and the Open University. Several of the Society's meetings have involved visits to museums and libraries, and every year ends with an enjoyable Christmas meeting featuring a mixed selection of talks proposed by the Society's President.

Like last year's Turing weekend, many BSHM meetings have commemorated historical anniversaries. Among these have been celebrations of Hamilton's quaternions (1843/1993), Hilbert's Paris lecture (1900/2000) and the four-colour problem (1852/2002), as well as events marking the births of Leonhard Euler (1707/2007), August Möbius (1790/1990), Charles Babbage and Michael Faraday (1791/1991), Karl Pearson (1857/2007) and John von Neumann (1903/2003), and the deaths of Robert Recorde (1558/2008), Gerolamo Cardano (1576/1976), Arthur Cayley (1895/1995), James Joseph Sylvester (1897/1997), P. G. Tait (1901/2001), G. H. Hardy (1947/1997) and R. A. Fisher (1962/2012).

\section{Joint meetings}

Like the 2012 residential meetings in Oxford, several of the Society's most successful meetings have been collaborations with other societies or institutions: early joint meetings included The history of mathematics education in England, 1800-1914 (with the British Society for the History of Science) and Mathematics, history and the distance learner (with the Open University). The year 2000 saw the beginning of a highly popular series of joint lectures with Gresham College (London) by distinguished historians of mathematics; these annual lectures can all be viewed on the Gresham College website (www.gresham.ac.uk).

In 2013, in addition to an afternoon of lectures on the History of computing at Gresham College, the Society's programme includes one-day joint meetings on Jacob Bernoulli (with the Royal Statistical Society), commemorating the tercentenary of his Ars Conjectandi, and Lewis Carroll, mathematician (with the Lewis Carroll Society and the Birmingham and Midland Institute).

Over the years the BSHM have held meetings jointly with the London and Edinburgh Mathematical Societies, the Mathematical Association, the Royal Meteorological Society, the Wellcome Trust, the Institute of Electrical Engineers, the British Societies for the History of Science, of Philosophy, and of Alchemy and Chemistry, and many other organisations.

\section{Speakers}

The BSHM has always tried to include a wide variety of speakers at its meetings - whether professional mathematicians, professional historians (of mathematics or otherwise), teachers, or popularisers of mathematics. Distinguished professional British mathematicians have included Sir Michael Atiyah, Sir Hermann Bondi and Sir Michael Berry, while the popularisers have included Ian Stewart and Simon Singh.

While he was President, Ivor Grattan-Guinness, a major figure on the international history of mathematics scene, emphasised the importance of having speakers from outside the UK, and since then European and American speakers (in particular) have featured regularly: the former have included Henk Bos (Netherlands), Evelyn Barbin and Jean Dhombres (France), Sergei Demidov (Russia), Umberto Bottazzini and Fulvia Furinghetti (Italy) and Eberhard Knobloch and Detlef Laugwitz (Germany), while speakers from America have included Karen Parshall, Joe Dauben, Wilbur Knorr, Claudia Zaslavsky and Benoit Mandelbrot.

\section{International conferences}

In 1997 the BSHM linked up with the Canadian Society for the History and Philosophy of Mathematics (CSHPM) for a joint conference at Oriel College, Oxford. This was followed, two years later, with a visit to York University in Toronto. Since then, three more joint meetings have taken place - at Clare College, Cambridge (2004), the University of Montreal (2007), and Trinity College, Dublin (2011). 


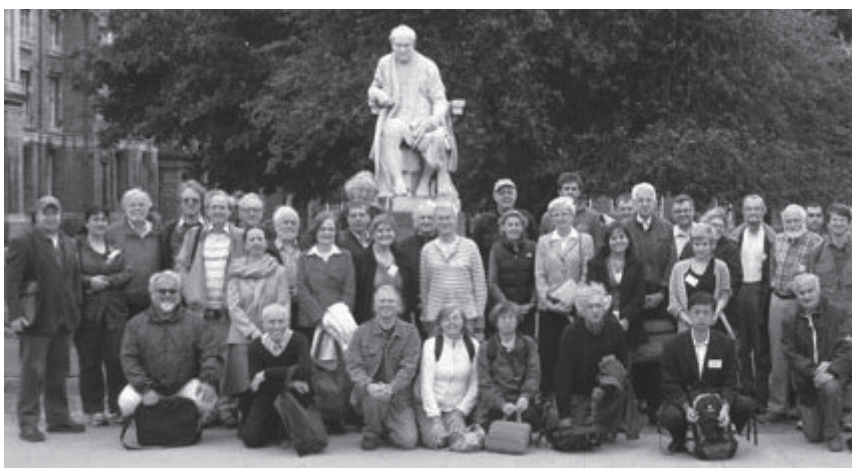

Participants at the BSHM-CSHPM Joint conference in Dublin in July 2011, watched over by Trinity College's former President and mathematician George Salmon. (Photograph by Tony Mann)

\section{HIMED and RiP}

Two of the BSHM's aims deal with education and outreach, and many of its meetings have been concerned with the use of history in the mathematical classroom, in both schools and universities. In the 1990s, inspired by the enthusiasm of John Fauvel, the Society arranged several 'HIMED' (HIstory of Mathematics in EDucation) meetings that brought together mathematicians, historians and teachers in a day or weekend of lectures and workshops. The first of these meetings, held in 1990 at Leicester University attracted 150 participants and was followed by similar meetings at Oxford, Nottingham and several other venues.

An annual feature of the Society's programmes for the past twenty years has been 'RiP', the Research in Progress meeting, at which graduate students in the history of mathematics from the UK and around Europe talk about their current researches in an informal and supportive environment. The consistently high quality of the presentations is inspiring to all, and the meeting is always one of the highlights of the year.

\section{Prizes}

For many years the BSHM has awarded history of mathematics prizes to authors and students. When the Open University's course MA 290: Topics in the history of mathematics was in existence (usually attracting about three hundred students per year), a prize was awarded, often at the Research in Progress meeting, to the student who performed best in the course. More recently the Society has awarded prizes for the best historical essay by a current full-time undergraduate at any university: recent prizewinners have written on 'The contribution of Évariste Galois to the founding of group theory', and 'The application of mathematical understanding in the ancient Olympic Games'

A different sort of prize is awarded every other year in honour of former BSHM President Dr Peter M. Neumann OBE, for a popular book on the history of mathematics. The first Neumann prizewinners (in 2009) were Reviel Netz and William Noel for The Archimedes Codex, and the 2011 prizewinner was Clifford Pickover for The Math Book.

\section{Publications}

In 1986 one of the members, Ronald Gowing, initiated a regular BSHM Newsletter in order to provide members with news of the Society's activities and other history of mathematics events in the UK and around the world. This three-page document rapidly increased in size, reaching 32 pages by 1992 and 68 pages for its 50th edition in 2004. By this time the current editor, Jackie Stedall, felt that it was high time to have a proper Society magazine, and the BSHM Bulletin, published by Taylor \& Francis, was born. This is a highly professional publication containing articles, BSHM news and book reviews.
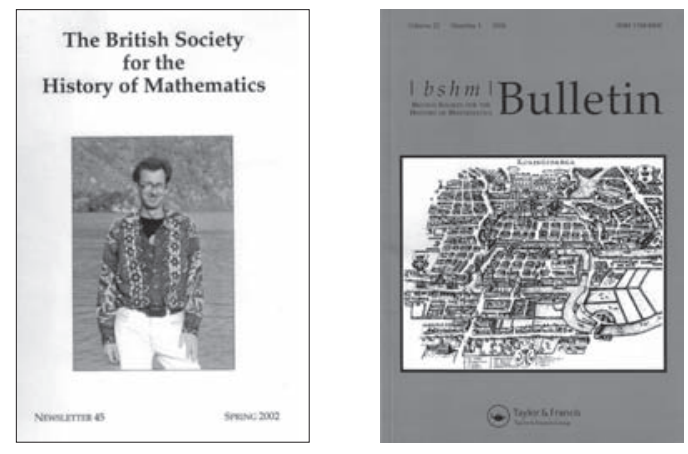

BSHM Newsletter 45 (2002) commemorating the life of John Fauvel, and the BSHM Bulletin Volume 23, No. 1 (2008).

We conclude with a tribute to the BSHM sent by Joe Dauben, the Chairman of the International Commission on History of Mathematics, in 1992:

As the BSHM begins its third decade as the only specialised society for history of mathematics in Europe, and the oldest such society in the world, its remarkable success reflects the growing seriousness of the study world-wide. In its first 21 years the society has sponsored more than 400 lectures by mathematicians, historians and philosophers of mathematics from many parts of the world, on subjects ranging from antiquity to modern mathematics. Issues relevant to mathematics education and, in particular, the role that history can and should play in teaching, have also been among its prime concerns.

As chairman of ICHM (a joint commission of the International Mathematical Union and the International Union for History and Philosophy of Science), I am especially pleased to convey this message of congratulations to the BSHM, with best wishes for its continued wellbeing and success. May it persist over the decades to come in promoting the history of mathematics, in as rich a diversity of ways as it has done in the past.

Further information about the BSHM and its activities can be found on its website: www.bshm.org.

\section{Acknowledgement}

We are very grateful to Ivor Grattan-Guinness for his excellent account, 'A tale of a tub: on the Society's first 21 years' in the BSHM Newsletter Number 21,1992; much of the material in this article is gleaned from this account. 


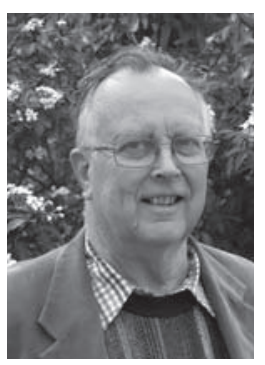

Robin Wilson[r.j.wilson@open.ac.uk] is Emeritus Professor of Pure Mathematics at the Open University (UK) and Emeritus Professor of Geometry at Gresham College (London), and currently teaches for Pembroke College, Oxford University, and the London School of Economics. He has written and edited over forty books, mainly on graph theory and combinatorics or the history of mathematics, and is involved with the popularisation and communication of mathematics and its history. He is currently President of the BSHM.

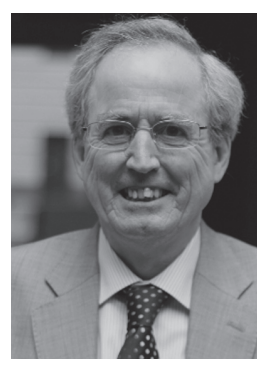

Raymond Flood[raymond.flood@kellogg.ox.ac.uk] was Vice-President of Kellogg College, Oxford University, and previously was University Lecturer in Computing Studies and Mathematics at the Department for Continuing Education, Oxford University. He is an Emeritus Fellow of Kellogg College. His main research interests lie in statistics and the history of mathematics, and he has written and co-edited several books. He was formerly President of the BSHM, and is currently Gresham Professor of Geometry.

\section{Hausdorff Research Institute for Mathematics (HIM)}

Wolfgang Lück (HIM - Hausdorff Research Institute for Mathematics, Bonn, Germany)

The Hausdorff Research Institute for Mathematics (HIM) organises international programmes devoted to topics in mathematics, mathematical economics and interactions of mathematics with other sciences, each of which lasts for four months. The institute was founded in 2006 and is part of the Cluster of Excellence "Hausdorff Center for Mathematics" at the University of Bonn. Bringing together a critical mass of expertise and providing an inspiring atmosphere, the activities at HIM initiate research at the forefront of mathematics in its full breadth. HIM gives scientists the opportunity to work on challenging projects, undisturbed and for longer periods, and to discuss them with leading experts in their fields. This leads to a substantial output of research results of the highest quality, initiated, pursued and sometimes even completed during the programmes.

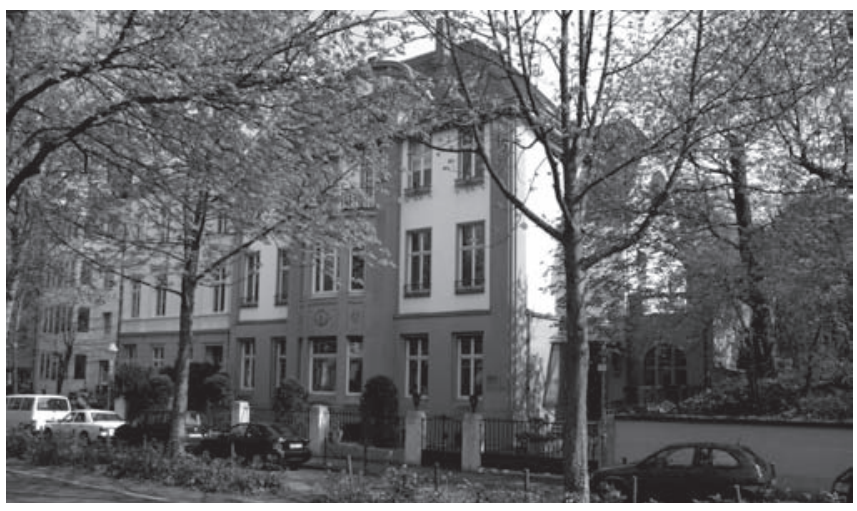

Main building

\section{Activities}

Generally, HIM organises two Trimester Programmes and one Junior Trimester Programme, each lasting four months. The programmes bring together leading experts and young talents from all over the world. HIM fosters longer term cooperation between the participants. Scientists from different fields or disciplines exchange their ideas and discuss problems. Ideally, a transfer of methods between different areas will lead to unexpected new results or solutions to problems in a field where the researchers had not been aware of suitable tools from other fields. These synergies occur when researchers have the opportunity to work together for extended periods, during which they become acquainted with the language and skills of other areas or disciplines, and establish new connections. Even new research directions are being created. The interactions at HIM have long-term effects and lead to cooperation continuing beyond the programmes.

- A Trimester Programme brings together scientists for extended periods to carry out research on a specific topic, often resulting in outstanding new achievements and new scientific cooperation. The institute can host up to 30 scientists at any one time and tries to distribute these places between senior mathematicians, postdocs and PhD students. Additional short-term visitors can be accommodated in the nearby annex. The theme of each programme is open to suggestion via proposals from groups of leading scientists. The proposals are evaluated by the members of the external Scientific Advisory Board who finally choose the theme. The overall topic can come from any area of mathematics, mathematical economics or mathematical physics.

The facilities and administration of HIM ensure that the programmes run smoothly in an informal, stimulating atmosphere. The organisers of a Trimester Programme decide on the final list of participants in agree- 


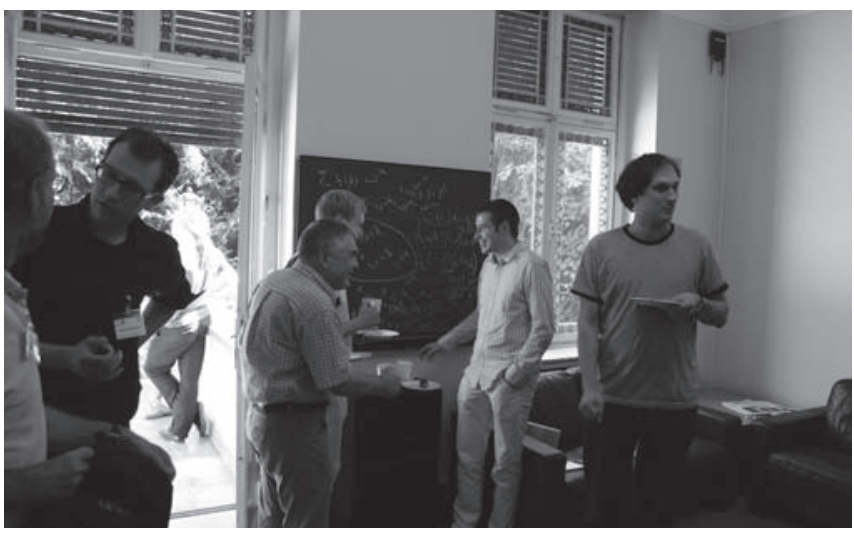

Tea time

ment with the director. This list is expected to represent a balance of age groups.

There is a preference for participants who stay for a longer period - this holds in particular for younger scientists. During the programme, participants can suggest researchers relevant for one of the research themes, who will then be invited for short visits by the director. Besides the selection of the best group of scientists, important factors for the success of a programme are specific activities like workshops, mini courses and reading seminars. These are very much encouraged and most programmes make good use of these opportunities. At the same time, the special culture and needs of the various mathematical areas and different disciplines are taken into account.

- Junior Trimester Programmes are specifically designed for promising young researchers by affording them the opportunity to develop an excellent research agenda independently of their advisors. In contrast to the regular Trimester Programmes which have a top-downstructure, the structure of the junior programmes is reversed. Here, young researchers constitute the core of the participants. They design the activities and programmes themselves and can suggest senior researchers to be invited.

HIM permits applications preferably by small groups but also by individuals whose research interests fit the current theme. Individuals should be open to project work with other participants.

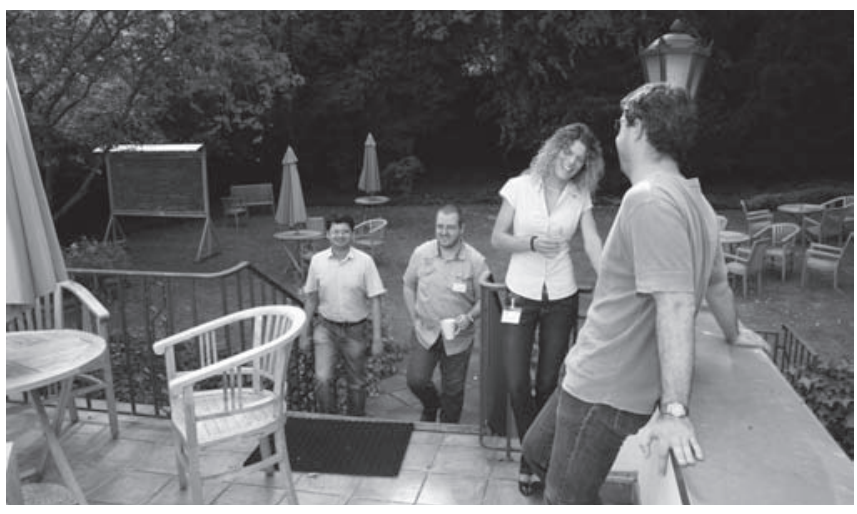

Patio

Cooperation with local Bonn mathematicians plays a particular role for the success of the Junior Trimester
Programmes. Ideally, the topics chosen for Junior Trimester Programmes are represented in Bonn, so that the Bonn faculty are available to coach and advise the young participants. The topics are chosen by the (external) Scientific Advisory Board.

- A new programme Research in Groups was initiated in 2009, giving small groups the opportunity to carry out cooperative research independent of the Trimester Programmes. This programme is rather flexible. It gives a group of at least three researchers the opportunity to carry out a very specific project or to discuss new directions of research. The duration of the programme can be up to three months and not shorter than one month (which in exceptional cases can be divided into two halves).

- HIM also sees its role as an interface between mathematics and the general public. It organises special activities on general topics (e.g. mathematics education in schools and universities) and public events.

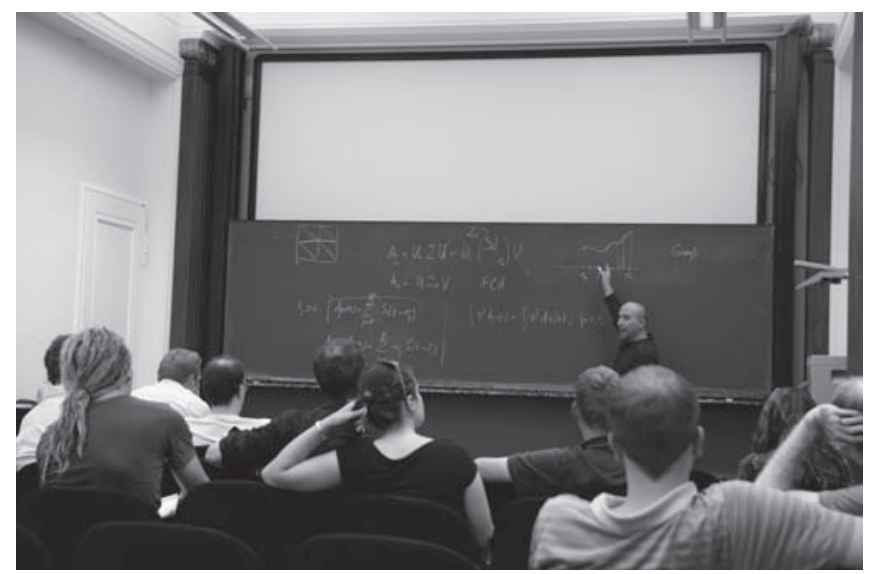

Lecture Hall

\section{The mathematical landscape}

HIM fills an important gap in the mathematical landscape of Germany, as no other institute in Germany offers international, long-term programmes in mathematics. In many other countries such institutions already exist. The combination of strong departments in mathematics and economics at the University of Bonn, the Max Planck Institute for Mathematics (Bonn) and HIM, which are within short walking distances of each other, as well as the focus on long-term activities, are unique features. HIM offers topic-oriented, long-term activities in contrast to the Max Planck Institute for Mathematics whose focus lies primarily on activities based on individual visitors. The programme structures of these two institutes are fundamentally different but they supplement each other in an ideal fashion. The Junior Trimester Programme at HIM is truly unique worldwide.

\section{Working environment and daily life}

HIM provides a stimulating atmosphere. The main building of HIM is a centrally located, attractive villa. It offers office space for 30 visitors. Almost half of the rooms are for single person occupancy. Each room is equipped with a blackboard and provides sufficient space for discussions with several participants. There are two lecture 


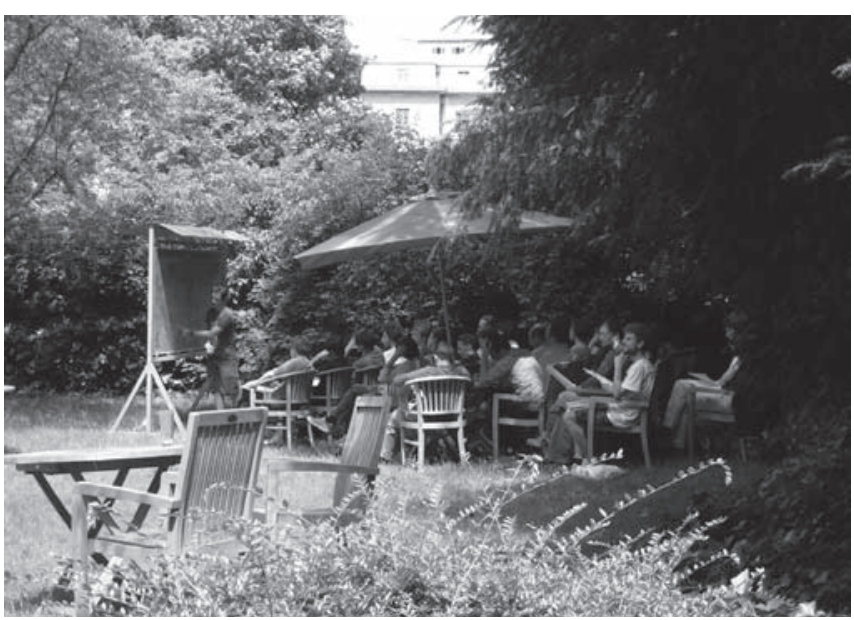

Lecture in the Garden

halls for up to 70 and 30 people, respectively. In addition, there are three beautiful meeting rooms. Even in the large garden a blackboard has been installed.

The nature of the programmes is very informal. There is a lot of freedom and flexibility for spontaneous initiatives by the participants. Interaction between the participants is encouraged and facilitated by the daily tea at 4 $\mathrm{pm}$. On these occasions, new contacts are established and many of the informal activities are initiated. It goes without saying that there is also ample opportunity to retreat and work for some time on one's own.

HIM rents and maintains about 30 apartments, which it sublets to its visitors. This is much more pleasant for the guests and, at the same time, less expensive than stay- ing in a hotel. Moreover, HIM is independent of seasonal events when hotels are overbooked and is also in the position to offer suitable housing for guests with families.

\section{A short review}

In the short time of its existence HIM has emerged as a successful research institute. Its programmes have covered a broad range of topics and it has hosted leading experts from all over the world. The organisers and guests have included both Fields Medallists and Nobel Prize winners. Numerous publications have been initiated or written during programmes at HIM. For detailed information on past programmes please visit the HIM website http://www.him.uni-bonn.de. This website also contains information on current and future activities.

\section{Call for Proposals}

The Hausdorff Research Institute for Mathematics calls for proposals for Trimester Programmes and Research in Groups. See http://www.him.unibonn.de/application/program-proposals/ for more information. Deadline for submission: 30 September every year.

\title{
ICMI Column
}

\author{
Mariolina Bartolini Bussi ((Università di Modena, Italy)
}

\section{First ICMI Study on Primary Schools}

The ICMI has launched the ICMI Study 23: "Primary mathematics study on whole numbers"

The International Programme Committee (IPC) includes Mariolina Bartolini Bussi (Italy, co-chair), Sun Xuhua (Macau-China, co-chair), Berinderjeet Kaur (Singapore), Hamsa Venkatakrishnan (South Africa), Jarmila Novotna (Czech Republic), Joanne Mulligan (Australia), Lieven Verschaffel (Belgium), Maitree Imprasitha (Thailand), Sarah Gonzales (Dominican Republic), Sybilla Beckmann (US) and Abraham Arcavi (ICMI Secretary General, ex-officio). The IPC, on behalf of the ICMI, is responsible for conducting the study.

The process of realising a study typically stretches over a period of about three to four years (http://www. mathunion.org/icmi/conferences/icmi-studies/introduction/). The rationale for this first study on primary schools is explained below.
Primary schooling is compulsory in all countries, although there are different facilities and opportunities for children to take advantage of it. One in three children never sees the inside of a classroom. Mathematics is a central feature of all primary mathematics education (the only such subject) and the delivery of that curriculum is important in all countries for the kinds of citizens each seeks to produce. Primary school mathematics education has been represented in other ICMI studies (for example, ICMI Study 9 on Geometry and Study 12 on Algebra); however, in most cases, secondary school mathematics education has dominated the ICMI studies. Hence, starting a reflection on primary school mathematics is timely.

In the international literature there is much research and writing on primary school mathematics. In many cases, especially in the West, early processes of mathematical thinking are investigated by cognitive psychologists, who study the emergence of these processes in laboratory set- 
tings, where children are stimulated to observe, for example, the emergence of one-to-one correspondence, counting or measuring. This is interesting but not sufficient to cope with the more complex situations of schools, with institutional and cultural constraints. In several countries (in Europe and North America) the influence of Piaget, although criticised in research literature, is still very deep and strong. In other parts of the world different models are adopted.

The study of this core content area is often regarded as foundational for later mathematics learning. However, the principles and main goals of instruction in the foundational concepts and skills are far from universally agreed upon, and practice varies substantially from country to country. An ICMI study that provides a meta-level analysis and synthesis of what is known about primary mathematics will provide a base from which to gauge silences and an opportunity to learn from different countries and contexts.

A reflection on primary mathematics education would not be complete without considering the context and conditions in which mathematics teaching and learning takes place. In developing countries, primary schooling for a vast majority of children may be continually interrupted, take place in overcrowded, under-resourced, large classrooms of substandard quality. A part of the study may be devoted to the analysis of some case studies. Primary mathematics curriculum policy reforms in different countries and the effectiveness of their implementation in classroom practice would also address a diversity of audiences.

The preparation of teachers for teaching mathematics in the primary grades is very important. In broad terms issues of lesson planning specific to primary mathematics has become emphasised through stronger prescription in some contexts. The literature on Lesson Study in Japan or Guan Mo in China provides another perspective that is relevant at this level. Another issue is that in many countries primary school teachers are generalists whilst in other countries they are specialists. Teacher education and development is expected to be present in this study.

The study will include a theme covering the approach to whole numbers, including operations and relations, the development of place value concepts and the solution of arithmetic word problems, in schools (and possibly preschool environments) up to grade 3 or more, according to the various education systems.

This area may be explored in terms of several subthemes:

a. What are the mathematical foundations of primary school approaches to whole numbers?

Where foundational processes (such as the development of number sense) are concerned, a strong epistemological basis is needed; this might be the added value of the ICMI (and the IMU through the ICMI) with respect to the analysis carried out in the field of cognitive psychology. Such epistemological analysis was part of the classical works of professional mathematicians (for example,
Klein, Smith, Freudenthal) who have played a big role in the history of the ICMI. For instance, it might be useful to focus on the following questions:

What is the conceptual significance of rote counting? How can a robust understanding of the operations of addition (and subtraction) be developed? How are arithmetic operations concepts articulated and enriched through word problems? How is the concept of place value developed? How can conceptual development of place value structure promote mental mathematics? How is it possible to develop an approach to arithmetic computation that intertwines place value with addition/ subtraction facts? How does "local" language, and in particular the linguistic aspects of the number names (regularity, modification according to context, etc.), affect the acquisition of number concepts and what are the implications for instruction?

b. What do we know about technologies and resources? What relationships exist between technologies/ resources and mathematics processes in primary school?

A wide interpretation of technology includes texts, blackboards and whiteboards, and learning resources (for example, abacus, suànpán and soroban as well as electronic technologies). Different cultures have different specific resources, sometimes embedded in language or indigenous practices. Different approaches are linked to different cultural traditions and to the constraints of the available materials and organisation. It is therefore necessary to analyse the availability, use and implementation of these various technologies.

c. What do we know about diagnosing specific learning disorders (e.g. dyscalculia, sensory impairments) that affect very basic arithmetic? What do we know about interventions that help students with such disorders? Learning disorders, such as dyscalculia, affect very basic arithmetic. It is the primary school teacher who first comes into contact with such learning difficulties. Analysing research on dyscalculia may help to reduce incorrect diagnoses that are actually the effects of ineffective teaching. We also need to understand research into rehabilitation, including the use of computer software. Another focus in this subtheme is teaching for, and learning by, students who are sight or hearing impaired. In each case different research communities exist with little or no interaction between them and with mathematics educators: neuropsychologists study learning disorders and special educators study primary school education for blind and deaf students. Results from specialist literature from both fields will be of interest and use to the mathematics education community.

\section{The project "Mathematics of Planet Earth 2013 (MPE2013)"}

Under the patronage of UNESCO, Mathematics of Planet Earth 2013 (http://mpe2013.org/) consists of holding a year of scientific and outreach activities on planetary issues. It will show the essential relevance of mathemat- 
ics to planetary issues. It is an extraordinary opportunity to develop interesting relevant material for teachers and schools and to share it with the rest of the planet.

Without claiming to be exhaustive, MPE2013 organisers have identified four subthemes:

- A complex planet. Earth is a planet with dynamic processes in the mantle, oceans and atmosphere creating climate. Mathematical sciences provide tools to understand these dynamic processes and measure the variation of their dynamics due to natural interaction or anthropogenic forcing.

- A biologically diverse planet. Earth is the home of life supporting systems which, through evolution, have generated biodiversity. Living species interact with ecosystems; new species appear or disappear; and species migrate to spread spatially. Mathematical modelling and analysis help us measure and understand what has happened in the past, predict what may happen in the future and manage the present to promote more desirable future outcomes.

- A planet structured by civilization. Humans have developed systems of great complexity, including those for communication, transportation, energy, water and health care. These man-made systems are the ones over which we exert the most direct control; however, we must often analyse massive amounts of data from monitoring systems, deal with multi-scale and/or distributed systems, and identify and quantify sources of uncertainty.

- A planet at risk. Human activity has increased to the point where it influences the global climate, impacts the planet's ability to feed its inhabitants and threatens stability of these systems. We need to understand how the Earth's systems interact. How does human activity affect climate? How does climate affect agriculture? How does agriculture affect water quality? How does the availability of food and water affect human health and migration and how does human migration and development affect animal migration? These are highly interconnected, complex adaptive systems. The mathematics of such systems is still poorly understood, while modelling them must draw expertise from many disciplines.

The partners in the initiative include many institutes and learned societies, including the ICMI. A call has been sent to the planet to join in. More information about ongoing activities in many countries (including European countries) is available at http://mpe2013.org/.

\section{Short news}

1) The new ICMI Executive Committee took office on 1 January 2013. As already announced, it includes Ferdinando Arzarello (Italy, President), Cheryl E Praeger (Australia, Vice-President), Angel Ruiz (Costarica, Vice-President), Abraham Arcavi (Israel, SecretaryGeneral), the following members-at-large: Catherine P. Vistro-Yu (Philippines), Jean-Luc Dorier (Switzerland), Roger Howe (USA), Yuriko Yamamoto Baldin (Brazil), Zahra Gooya (Iran), and the following ex- officio members: Bill Barton (Past President of the ICMI), Ingrid Daubechies (President of the IMU) and Martin Groetschel (Secretary of the IMU).

2) Some meetings organised by the ICMI affiliate organisations http://www.mathunion.org/icmi/about-icmi/ affiliate-organizations/introduction/ will take place in 2013 in Europe. A list follows with websites:

ERME (European Society for Research in Mathematics Education), Antalya (Turkey), 6-10 February 2013, http://cerme8.metu.edu.tr/.

CIEAEM (International Commission for the Study and Improvement of Mathematics Teaching), Turin (Italy), 22-26 July 2013, http://www.cieaem. org $/$ ? $\mathrm{q}=$ node $/ 30$.

PME (International Group for the Psychology of Mathematics education), Kiel (Germany), 28 July-2 August, http://www.pme2013.de/en.

Moreover, for researchers interested in primary mathematics education:

SEMT (International Symposium on Elementary Mathematics Teaching), Prague (Czech Republic), 18-23 August, http://www.semt.cz/.

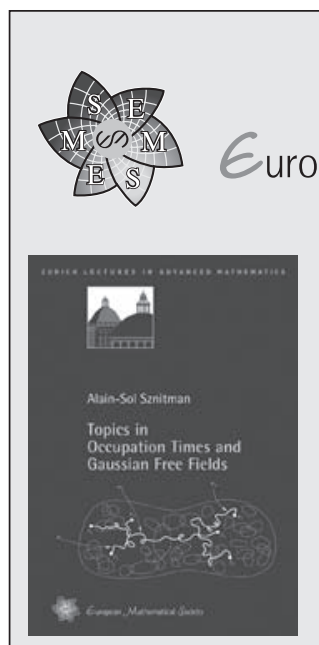

uropean Mathematical Society random interlacements, which have been the object of intensive research over the last few years. These notions are developed in the convenient set-up of finite weighted graphs endowed with killing measures.

The book first discusses elements of continuous-time Markov chains, Dirichlet forms, potential theory, together with some consequences for Gaussian free fields. Next, isomorphism theorems and generalized Ray-Knight theorems, which relate occupation times of Markov chains to Gaussian free fields, are pre- sented. Markovian loops are constructed and some of their key properties derived. The field of occupation times of Poisson point processes of Markovian loops is investigated. Of special interest are its connection to the Gaussian free field, and a formula of Symanzik. Finally, links between random interlacements and Markovian loops are discussed, and some further connections with Gaussian free fields are mentioned.

European Mathematical Society Publishing House

Seminar for Applied Mathematics

ETH-Zentrum SEW A27, CH-8092 Zürich, Switzerland

orders@ems-ph.org / www.ems-ph.org 


\section{Solid Findings in Mathematics Education: Living with Beliefs and Orientations - Underestimated, Nevertheless Omnipresent, Factors for Mathematics Teaching and Learning}

Günter Törner (University of Duisburg-Essen, Germany) for the Education Committee of the EMS

\section{Triggers}

Already in the 1940s researchers were investigating the question of whether attitudes may have some influence on the reception of mathematics and its assessment. Articles on this subject provide an affirmative answer, which may be astonishing for an expert. In addition to the rather obvious affective side, it was learnt in the 1950s that, even more, subjective understandings of mathematics and mathematics teaching are influencing the reception of the content and may lead to an unsatisfactory assessment of mathematical topics. Even more, they may affect engagement structures (see Goldin et al., 2011).

In 1983, Alan Schoenfeld published an article dealing with important driving forces beyond the purely cognitive. His insights contributed to a growing research field, whose phenomena were not well understood but accepted as observed and highly influential. Schoenfeld pointed out two years later: These phenomena were, on the whole, negative; for example students were turned off mathematics by the drill-and-practice during the heyday of the back to basics movement.

For a short while, it seemed that propagating problem solving as a curricula guideline would be a promising alternative. However, curricula favouring problem solving, first established in the USA in the 1980s, soon turned out to be a failure. What were the reasons?

It was Marta Frank who studied in her PhD (1985) the (potential) incompatibility between problem solving and teachers' and students' inherent beliefs.

What are beliefs? Referring to a definition of Schoenfeld: Beliefs are mental constructs representing the codification of people's experiences and understandings. Beliefs have already been studied by educational psychologists. It was Green (1971) who emphasised that by communicating knowledge, to shape behaviour, e.g. doing mathematics, the instruction and teaching processes are always accompanied by generating and shaping beliefs. Beliefs are not isolated, arbitrary subjective constructs; they are part of larger systems. To quote Green (1971): Nobody holds a belief in total independence of all other beliefs (p. 41). Beliefs always occur in sets or groups. Beliefs are clustering. So the terminology used by Schoenfeld and offering the term mathematical worldviews is figurative.
Thus, we have to accept mental subjective structures beyond the purely cognitive, which are widely unknown and not easily influenced. To be precise, Frank and many others stated that students may not be able to become better problem solvers unless they change their beliefs about mathematics and build up adequate worldviews on mathematics.

As Green (1971) pointed out, beliefs are not inherent only in mathematics education. Beliefs occur everywhere; we are living by beliefs, often not being aware of beliefs that may occur e.g. as prejudices. In particular, as shown below, in research mathematics much is also based on beliefs.

\section{Folklore beliefs}

We list some folklore beliefs, which can be encountered in nearly all mathematics classrooms across the world and which are still alive despite many teachers gradually becoming aware of these misleading views and fighting against them.

- "Mathematics is created only by very prodigious and creative people; other people just try to learn what is handed down."

- "One can not engage in doing mathematics by oneself. One requires instruction."

- "Mathematics is unjust, because some students are privileged, while most are at a disadvantage."

- "Dependence on the teacher is important in mathematics. However, if the teacher cannot 'clarify', there is little chance that the student will understand mathematics."

- "Deduction is of the highest importance in mathematics. Thus structures and proofs play a major role in mathematics."

- "There is, in this sense, nothing worse than making mistakes in mathematics. Mistakes are damned."

- "A mathematical task always has only one solution, so I hate mathematics, because there are thousands of wrong answers, however only one correct answer."

The last quotation from a student's interview (age 10) makes clear that inadequate or insufficient worldviews on mathematics influence our attitude towards mathematics directly. 
- "Any mathematical problem can be solved in two minutes, or not at all."

Again this restricted understanding on mathematical problems partly explains the failure of the innovative approach of problem solving. Again we have to ensure in advance that the beliefs about mathematics as a whole are not misleading. Research has proven that these beliefs of that type, sometimes called estimations, assumptions, prejudices and half-known knowledge, drive the thinking of students in many classrooms around the world.

But not only the behaviour of students leaves something to be desired; the same laws apply to teachers and again many folklore beliefs can be found in various papers. We refer to an article of Tobin and LaMaster (1992) which states: However, what became apparent was that teachers implemented the curriculum in accordance with their own knowledge and beliefs and did not necessarily do what curriculum designers envisioned. Several studies ... indicated that teachers do what they do in classrooms because of their beliefs about what should be done and how students learn. (p.115)

\section{Beliefs in research mathematics?}

At first glance, the reader might accept our statements but be reluctant to accept a similar situation also in the field of mathematics. To be honest, our research is often driven by other research and there is nothing sinister in this. But some of the beliefs on mathematics are rather global, among them philosophies. Platonism, for example, is a belief, a worldview or a philosophy; the same is true about formalism. Hersh (1997) describes devastating influences of certain philosophies. In an introductory paper of P. J. Davis (1972), he lists the various, often non-reflected implications of a 'platonic mathematics philosophy'. Mathematicians are sometimes disputing different underlying philosophies; however other philosophies (see Hersh, 1997) imply different grounded assertions and worldviews.

It should not be ignored that philosophies about mathematics are also influencing our pedagogy, an effect René Thom (1973) phrased in the following way: In fact, whether one wishes it or not, all mathematical pedagogy even if scarcely coherent, rests on a philosophy of mathematics (p. 204). Although Thom's statement has to be acknowledged as a hypothesis from an educational perspective, there is much evidence for the linkage between mathematics and mathematics education.

To use the terminology of belief research, philosophies about mathematics can be understood as beliefs about the (large) 'belief object' mathematics. But there are also beliefs about smaller objects; take, for example, the definition of continuity.

We all know that these beliefs, the associations or the students' 'concept image' are often not adequate for explaining some difficulties in our university classrooms. But the list of examples is endless: think of vector spaces, differential equations or skew fields, just to mention three mathematical objects or terms. Think of the formula $z^{n}=r^{n}(\cos n \varphi+\mathrm{i} \sin n \varphi)$ with $z$ a complex number; dis- cuss it with your students and you will realise that there are beliefs associated and, very often, emotions or affections linked to them. Finally, use a 'smaller' belief object, namely the equation $(-1)(-1)=1$ and again you will encounter beliefs. Feel encouraged to start a discussion on this equation with students at school. You will experience what a researcher once described with the metaphor: Investigating beliefs is like picking out a single spaghetti thread and soon you will realise that more threads are glued to it.

\section{Research on beliefs and orientations}

What should be learnt? Where do we stand today? What is worth being mentioned?

Beliefs are no longer a hidden variable acting influentially as they were described at the beginning of the century (Leder, Pehkonen, Törner, 2002). Most mathematics education researchers are aware of beliefs. While in 1992 a first survey article by Thompson about the influence of mathematics teachers' beliefs was published, today every handbook contains a chapter dealing with beliefs - and each international conference has its beliefs session.

Nevertheless, 'beliefs' are a fuzzy construct and it seems unsatisfactory to mathematicians that there is generally no internationally accepted definition. Thus, when reading a paper dealing with beliefs, you often have to question what the author's (personal) definition on beliefs is. However, Pajares (1992) stated that the most fruitful concepts are those to which it is impossible to attach a well-defined meaning (see an analysis in Törner, 2002). Thus, different authors are often using slightly different 'definitions'.

Meanwhile, Schoenfeld (2010) has introduced a new term, namely orientations, which is a much wider construct and which is also integrating individual goals that are closely connected with one's beliefs. Beliefs and goals often explain themselves mutually.

There is still a dispute in the community of whether we should include affections and emotions within the definition of beliefs. Both alternatives have their 'pros': nearly all beliefs are linked to observable emotions and affections in different degrees; on the other side, the theories of emotions and affections, partly including attitudes, follow different patterns from those on beliefs.

Recently, Goldin et al. (2011) pointed out that socalled engagement structures - for an initial understanding refer to the standard meaning of 'engagement' - can be traced to beliefs and worldviews. However, it is still an unanswered question in educational psychology and sociology whether actions are induced by beliefs and connotations should be associated. Believing and acting accordingly is not a general rule. But the opposite is also not true; very often, actions can be explained through the beliefs of an individual.

\section{What are the implications of belief theory for the teaching and learning of mathematics?}

Although mathematics is respected as a theory which does not accept any tolerance in terminology and conceptions, we are living by beliefs. We need a finite set of 
beliefs since they restrict our world to a finite number of patterns. In some cases beliefs are productive and they open our thinking but often they hinder our thoughts and serve as inertia obstacles. Thus we should be aware and sensitive of beliefs beyond the purely cognitive landscapes in mathematics. Nevertheless, we can often rationally explain students' behaviour in a mathematical context because of their underlying beliefs. They are also responsible for our orientations, which are closely linked with goals (see Schoenfeld, 2010): If you are believing something, you will strive for it and declare it as your goal in action and vice versa.

To change beliefs is a hard job and there is no royal road to being successful. How far beliefs determine our behaviour and connotations is still an open question in social psychology. Nevertheless, there are indications that inadequate beliefs may lower the assessment of students while doing mathematics. To prove this empirically is a really hard job since we have to distinguish between proclaimed and possessed beliefs. However, there is a lot of smoke so there must be some fire somewhere.

Thus, generating and influencing adequate beliefs (which can be extended - if it is necessary - at a later time) is decisive. In mathematics, we do not only have to promote knowledge but also handle and develop beliefs, hopefully to induce adequate beliefs which are productive.

\section{Beliefs - why do we estimate them as solid findings?}

In our first article on solid findings in 2011 (EMS Newsletter Issue 81) we listed some criteria for using the quality label of 'solid findings' for the construct under discussion. There is a large body of evidence that all criteria are fitting. Beliefs are not a result of a single research initiative; thousands of articles have proved the 'existence' of beliefs and their influence on teaching and learning of mathematics. Thus, the trustworthiness has to be accepted. Beliefs are an excellent construct in mathematics education to show how universally this idea can be applied. Although beliefs may be dependent on the cultural framework or setting and they may differ in different cultures, they can be verified. Beliefs have also deepened our understanding since they serve as an additional variable, which was hidden before. And as it is happening in many contexts, the more we know about beliefs, the more questions will be asked, e.g. the highly complex structures of how beliefs are influencing our behaviour and our decisions.

\section{References}

Davis, P. J. (1972). Fidelity in Mathematical Discourse: Is $1+1$ Really 2 ? Amer. Math. Monthly 78 (3), 252-263.

EMS-Committee of Education. (2011). 'Solid Findings' in Mathematics Education. EMS-Newsletter September 2011, pp. 46-48.

Frank, M. L. (1985). Mathematical beliefs and problem solving. Doctoral dissertation. Purdue University, West Lafayette (Ind.), University Microfilms International.

Green, T. F. (1971). The Activities of Teaching. Tokyo: McGraw-Hill Kogakusha.
Goldin, G.; Rösken, B.; Törner, G. (2008). Beliefs - no longer a hidden variable in mathematical teaching and learning processes. In Maaß, J.; Schlöglmann, W. (Eds.) Beliefs and Attitudes in Mathematics Education (pp. 9-28). Rotterdam: Sense Publishers.

Goldin, G., Epstein, Y., Schorr, R. \& Warner, L. (2011). Beliefs and engagement structures: Behind the affective dimension of mathematical learning. ZDM Mathematics Education 6, 547-560.

Hersh, R. (1997). What is Mathematics, Really? New York: Oxford University Press.

Pajares, M. F. (1992). Teachers' beliefs and educational research: Cleaning up a messy construct. Review of Educational Research 62 (3), 307-332.

Schoenfeld, A. H. (1983). Beyond the purely cognitive: Belief systems, social cognitions, and metacognitions as driving forces in intellectual performance. Cognitive Science 7, 329-363.

Schoenfeld, A. H. (2010). How we think - a theory of goal-oriented decision making and its educational applications. New York: Routledge.

Thom, R. (1973). Modern mathematics: does it exist? In A. G. Howson (Ed.), Developments in Mathematical Education. Proceedings of the Second International Congress on Mathematical Education (pp. 194-209). Cambridge: Cambridge University Press.

Thompson, A. G. (1992). Teachers' beliefs and conceptions: A synthesis of the research. In D. A. Grouws (Ed.), Handbook of research on mathematics learning and teaching (pp. 127-146). New York: Macmillan Publishing.

Tobin K.; LaMaster, S. U. (1992). An Interpretation of High School Science Teaching Based on Metaphors and Beliefs for Specific Roles. In E. W. Ross, J. W. Cornett \& G. McCutcheon (Eds.), Teacher personal theorizing: Connecting curriculum practice, theory and research. Albany: SUNY Press.

Törner, G. \& Pehkonen. (1996). On the structure of mathematical belief systems. International Reviews on Mathematical Education (ZDM) 28 (4), 109-112.

Törner, G. (2002). Mathematical beliefs - a search for a common ground. In Leder, G. C.; Pehkonen, E. \& Törner, G. (2002). Beliefs: A Hidden Variable in Mathematics Education? (pp. 73-94). Dordrecht: Kluwer Academic Publishers. 


\section{Current Issues on Mathematics Education Around Europe}

\section{Introduction}

In all the meetings of the EMS-Educational Committee, updated reports are presented by the participants on the current situation related to mathematics education in their countries. Although these reports express the members' personal opinions and do not necessarily reflect national institutional views, they address important issues that provide an initial comparative picture of mathematics education in Europe. The countries that are represented in the reports are Austria, Belgium, Czech Republic, Denmark, England, Finland, France, Germany, Greece, Israel, Italy, Portugal, Russia and Spain. In this short paper, the discussion will be around four main aspects that have been addressed in most reports, concerning: the overall educational and social matters that have an effect on mathematics education in different educational levels; the curriculum; teacher education and professional development; and finally the role of local associations, research groups and national projects, which is also a factor that seems to influence mathematics education at the levels of both research and practice.

\section{Social and Educational Issues}

Financial crisis around Europe, especially in some countries (e.g. Greece, Italy, Portugal, Spain), has resulted in salary cuts in the public sector and a number of educational changes (e.g. merging of schools and universities, reduction of funding, increase of the number of students in the classroom), each of which have had a negative impact on many teachers' motivation. In many countries, for example in France, Denmark, England, Israel and Germany, there is a shortage of school mathematics teachers for all or for some levels of educations. In some cases, where mathematics teaching is undertaken by out-of-field-teachers, there is the problem of low mathematical background even if their engagement is not problematic. In France, another reason that seems to create unwillingness for graduates to choose the teaching profession is the sheer number of challenges: the recent reform requires that they must simultaneously get a Master's degree, succeed in the national competition for teacher recruitment and teach fulltime in schools with no professional preparation.

In general, the public image of mathematics seems to be on a negative trend, with the number of university mathematics graduates decreasing dramatically and many mathematics departments having major difficulties in attracting students, for example in Spain, Denmark and Russia. An exception to the negative trend in mathematics recruitment at higher levels in school and into mathematics undergraduate courses is England where numbers have risen year on year over several years. Even in countries with strong mathematical traditions such as Russia, the positive image deteriorates. In Russia, mathematics education works at two different levels: at a very high level in specialised maths-physics schools and at some universities and at a much lower level elsewhere. Finally, politicians in most countries are keen to see a rise in students' achievements in international comparative studies (like PISA and TIMSS) and this has an impact on mathematics education in the country. For example, a number of educational innovations (e.g. new mathematics curricula, in-service teacher education programmes, EU-wide dissemination programmes like Fibonacci and PRIMAS) have been designed to try to improve outcomes in these studies.

\section{Curriculum Innovations}

At the school level, new curricula have been designed recently or are in the process of development in the Czech Republic, Denmark, England, Greece, Israel, Portugal and Russia. For example, in the Czech Republic a new curriculum was introduced first for compulsory education and then for upper secondary level. This curriculum is currently being evaluated and necessary modifications proposed. In Denmark, new curricula for primary and lower secondary levels and for the different streams of the upper secondary level were put in place 2005-2010. The changes of the general stream of the upper secondary level were rather marked, breaking with the tradition in several respects, e.g. by giving considerable emphasis to applications and to the role of CAS calculators in executing mathematical processes, while manifestly reducing the role of mathematical proof. This has given rise to quite some discussion amongst mathematics educators, teachers and mathematicians. In Israel, new pre-school and primary school mandatory curricula were implemented a number of years ago. A new junior high school curriculum is currently being implemented and revised; one of its characteristics is the stress on applied settings. A committee of leading teachers, teacher educators, academics and education ministry representatives is currently designing a new high school curriculum at four levels: theoretical, scientific, general and applied. In England a new draft National Curriculum is under consultation with quite radical changes proposed particularly for the primary phase with a return to more "formal methods".

In most countries, mathematics education at the compulsory level emphasises problem solving, reasoning and communication and the idea of developing students' mathematical literacy. Debates take place between mathematicians and mathematics educators in some countries 
about the quality of curriculum concerning mathematics and pedagogy. In the interests of openness and honesty, we cannot ignore that in some places there is a struggle between these two groups, in some cases even to the point of "maths warfare". Sometimes, information on both sides is lacking. An interest of our committee is in fact to improve communication. At the university level, in all the countries except Russia and partly Denmark, the universities design their own study programmes and some attempts have been made to improve mathematics teaching.

Developing educational standards and new examination systems has also been a goal for many European countries. For example, in Austria educational standards have been developed and field tested for grades 4 and 8 for a number of school subjects including mathematics (in 2012, the testing started with M8). In Germany there have been intensive discussions on standards of mathematics teaching and models of competencies in mathematics have been successfully established and discussed. In Russia an important development is the nationwide implementation of the Unified State Examination (USE) in mathematics (and other subjects). The USE has two functions: first, to control and unify the high school diploma examinations and, second, to serve as the main criterion for entering university (thus replacing the entrance exams organised by the universities themselves in previous years). This year, the selection of mathematics problems at the USE was generally regarded as very appropriate; nevertheless there is a strong feeling in the mathematics education community that the USE should be replaced by two separate examinations, each performing one of the two functions mentioned above. Changes to entry examinations at university have been planned or are being designed in many other countries such as Austria, Czech Republic, Germany and Greece.

Some other examinations changes are the introduction of standardised tests and the organisation of these examinations centrally (fully or partially) at a national level. In England there has been national testing at ages $7,11,14$ and 16 years and monitoring across core subjects including mathematics for many years. Interestingly this huge edifice of testing is now gradually being reduced (to date national tests at ages 7 and 14 have been discontinued). The number of school years at which national tests for school mathematics are organised varies across countries. In Spain national testing is more narrowly practised, for a lower number of years and only for compulsory mathematics education. In the Netherlands, there is an ongoing public debate about the direction of the mathematics curriculum of the future, especially at the elementary school level, between advocates of reformbased mathematical curricula and approaches (who are strongly inspired by the ideas and materials developed by the Freudenthal Institute) and advocates of more traditional, back-to-basics approaches.

\section{Teacher Education and Professional Development}

The issue of teacher education and professional development has been a central issue of educational policy in many countries. Five European countries (Germany, Norway, Russia, Spain and Switzerland) participate in the international study TEDS-M that aims to examine how teachers are prepared to teach mathematics in primary and lower secondary school (http://teds.educ.msu. edu). Teacher education is usually organised at university or at college level and differs for primary and secondary school teachers. For example, in Portugal the path for primary school teaching is through study in special schools belonging to the Polytechnical system, where the focus is on the study of all subjects and their didactics. At the secondary level, the prospective teachers study three years of mathematics followed by two years of courses with an emphasis on mathematics, didactics of mathematics and fieldwork. A similar situation exists in Belgium, although recently some initial steps have been taken in the direction of requiring an academic Master's degree for all future teachers. In Denmark the education of primary and lower secondary teachers takes place in teacher training colleges and not at universities. The programme for these prospective teachers is defined by the parliament and, due to continuing dissatisfaction with the outcomes of primary and lower secondary teacher education, the government and the parliament keep changing the programme, often even before the previous programme is put into full practice. At the upper secondary level, even though a Master's degree in mathematics and another subject is required, the lack of teachers for this level has implied a reduction of this requirement in practice. The situation in Israel is similar to the one in Denmark. In Spain, at secondary level an extra requirement to become a school teacher has been introduced that is to undertake a one year Master's programme designed by mathematics and education departments in collaboration. In Greece, a new law has been introduced that a teaching certificate is required for teaching at school and the mathematics departments have started to discuss organising courses leading to this certificate. In England there are many routes into teaching - from school-based courses run by schools (with university accreditation) to one year postgraduate courses at universities. The training for primary teachers is generalist but given the acknowledged importance of mathematics there are moves to introduce more mathematics specialism. In Austria, there are recent innovations in teacher education with an emphasis on the establishment of education infrastructure that would support teachers both at the pre-service and in-service level. For example, a number of regional networks for teacher collaboration and six national and eighteen regional subject-didactics centres have been established (IMST project). In Germany, a German Centre for Continuous Professional Development (DZLM) for mathematics teachers has been established, adopting some ideas from the successful National Centre of Excellence of Teaching Mathematics (NCETM) in England and from the establishment of centres and networks as initiated by the Austrian IMST project.

Another issue that seems to be of concern for many countries is teachers' mathematical knowledge, especially at primary level. For example, some preliminary re- 
sults from the TEDS-M Study show the need to reinforce primary school teachers' mathematical knowledge. Also, in a recent report of the Dutch Royal Academia of Sciences, the need to enhance elementary school teachers' mathematical content knowledge, as well as their pedagogical content knowledge, is mentioned as one of the greatest challenges for the improvement of the quality of elementary school mathematics (http://www.knaw.nl/ smartsite.dws? $\mathrm{id}=26103 \& \mathrm{pub}=20091080$ ). This is particularly recommended in countries like Spain in which primary teacher education programmes have been proven to give priority to the teaching and learning of general pedagogical content.

\section{Associations - Research Groups - National and International Projects}

In many European countries, mathematical and mathematics education associations and research groups are very active in collaborating with teachers, organising national conferences for teachers and taking part in reforms and national projects (e.g. France, Portugal, Spain and the Netherlands). A number of National Centres have been established that support education in general and mathematics education in particular. In Austria, six Austrian Educational Competence Centres have been established and are involved in current educational developments. In Israel, two National Teacher Centres are active, one for primary schools and the other for secondary schools; they offer in-service training and resources like learning activities and ICT tools, publish teacher journals, translate relevant scientific articles and maintain the contact between teachers and the education ministry. In Finland and in Germany (see above), a mathematics and science centre is also established that organises various activities for promoting the image of mathematics to students, teachers and parents such as special days at school, scientific clubs and teacher training courses. Finally, some European projects such as PRIMAS (www.primas-project.eu) and Fibonacci (http://www.fibonacci-project.eu) support mathematics teachers' professional development. We would like to be aware of further joint European projects and initiatives. In England, The National Centre for Excellence in the Teaching of Mathematics (NCETM) was set up in 2006 by the UK government and has a contract to continue to March 2015. The Centre aims to meet the professional aspirations and needs of all teachers of mathematics through evidence-based CPD provision. The National Centre encourages schools to learn from their own best practice through collaboration among staff and by sharing good practice locally, regionally and nationally. These collaborations take place face-to-face at national and regional events and in local network meetings across England or, virtually, through interactions on the NCETM portal, www.ncetm.org.uk.

\section{Authorship}

Even though certain authors have taken the lead in each article of this series, all publications in the series are published by the Education Committee of the European Mathematical Society. The committee members at the time of writing this report were Ferdinando Arzarello, Tommy Dreyfus, Ghislaine Gueudet, Celia Hoyles, Konrad Krainer, Mogen Niss, Jarmila Novotná, Juha Oikonnen, Núria Planas, Despina Potari, Alexei Sossinsky, Peter Sullivan, Günter Törner and Lieven Verschaffel.

\section{$\mathrm{CRM}^{\mathrm{N}}$

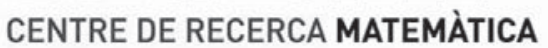

CENTRE DE RECERCA MATEMÀTICA

Bellaterra, Barcelona

\section{CALL FOR RESEARCH PROGRAMMES}

The Centre de Recerca Matemàtica (CRM) invites proposals for Research Programmes for the academic year 2015-2016.

CRM Research Programmes consist of periods ranging between two to five months of intensive research in a given area of mathematics and its applications. Researchers from different institutions are brought together to work on open problems and to analyse the state and perspectives of their area.

Guidelines and application instructions can be found at http://www.crm.cat/en/Pages/DetallCrida.aspx?ItemID=7

The deadline for submission of proposals:

OCTOBER 25, 2013 for the preliminary proposal

NOVEMBER 29, 2013 for the final proposal 


\section{The Software Information Service swMATH - Release of the First Online Prototype}

Sebastian Bönisch, Gert-Martin Greuel and Wolfram Sperber (all FIZ Karlsruhe, Berlin, Germany)

In March 2012, we reported about a project which deals with creating a comprehensive open access database for mathematical software. ${ }^{1}$ In the meantime, the project has considerably evolved and we are glad to announce the release of the first productive online prototype of swMATH. While the aforementioned article mainly explained the ideas and methods underlying the swMATH service, we will now focus on the prototype web application and describe its features in detail.

\section{Overview of the swMATH Project}

But let us start with a brief overview of the swMATH project and recall the key facts and main ideas.

The goal of the swMATH project is to create a novel information service for mathematical software. swMATH is a joint project of Mathematisches Forschungsinstitut Oberwolfach (MFO) $)^{2}$ and Fachinformationszentrum Karlsruhe. ${ }^{3}$ It is scheduled for three years (2011-2013) and is funded by the Leibniz Association. ${ }^{4}$ Several institutions with proven expertise in the field of mathematical software are supporting the project: Konrad-ZuseZentrum für Informationstechnik Berlin (ZIB), ${ }^{5}$ DFG Research Center MATHEON, ${ }^{6}$ Weierstrass Institute for Applied Analysis and Stochastics (WIAS) ${ }^{7}$ and FelixKlein-Zentrum für Mathematik ${ }^{8}$ in Kaiserslautern.

swMATH not only offers open access to a comprehensive database with information on mathematical software but also provides a systematic collection of references to software-relevant publications. For each software package contained in swMATH, references to peer-reviewed mathematical publications are provided which refer to this software; in particular, all such articles referenced in Zentralblatt $\mathrm{MATH}^{9}$ are listed. By systematically linking mathematical software and publications, swMATH provides the potential software user with information on the actual usage of certain software which is hard to find elsewhere. Moreover, software developers benefit from the systematic collection of literature references to their work by an independent institution and

\footnotetext{
1 "Building an Information Service for Mathematical Software - the SMATH project", EMS Newsletter 83, pp. 51-52.

2 http://www.mfo.de.

3 http://www.fiz-karlsruhe.de.

4 http://www.wgl.de.

5 http://www.zib.de.

6 http://www.matheon.de.

7 http://www.wias-berlin.de.

8 http://www.felix-klein-zentrum.de.

9 http://www.zentralblatt-math.org.
}

can gain interesting insights about the dissemination and the usefulness of their software.

\section{The swMATH Prototype}

In September 2012, we presented the first online prototype of swMATH at the Annual Meeting of the DMV (German Mathematical Society) in Saarbrücken. Since then, the prototype has been constantly improved and enhanced. The most current version of swMATH can be found at any time at:

http://www.swmath.org.

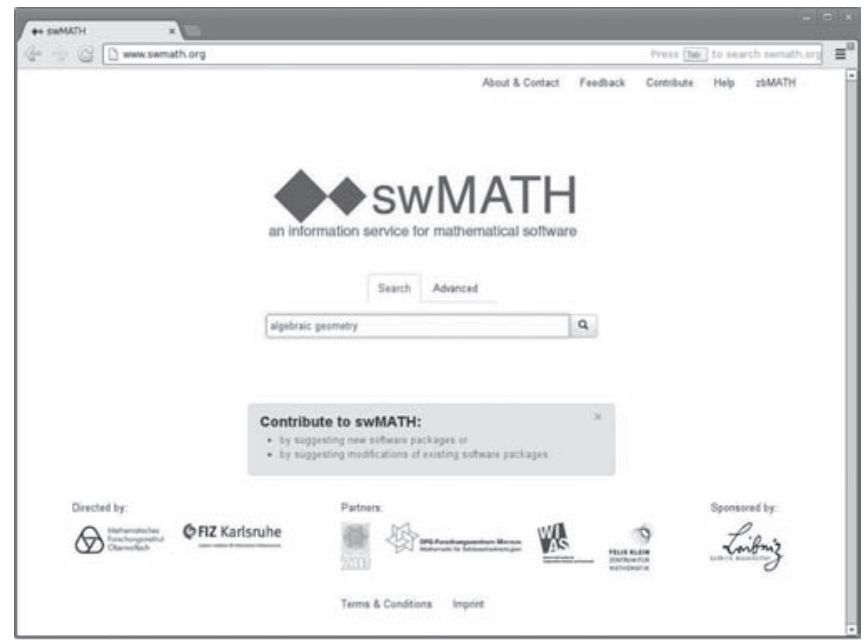

Fig. 1. The swMATH homepage.

The swMATH homepage welcomes the user with a simple, Google-like search interface (see Figure 1). An advanced search mask can be used to issue more specific

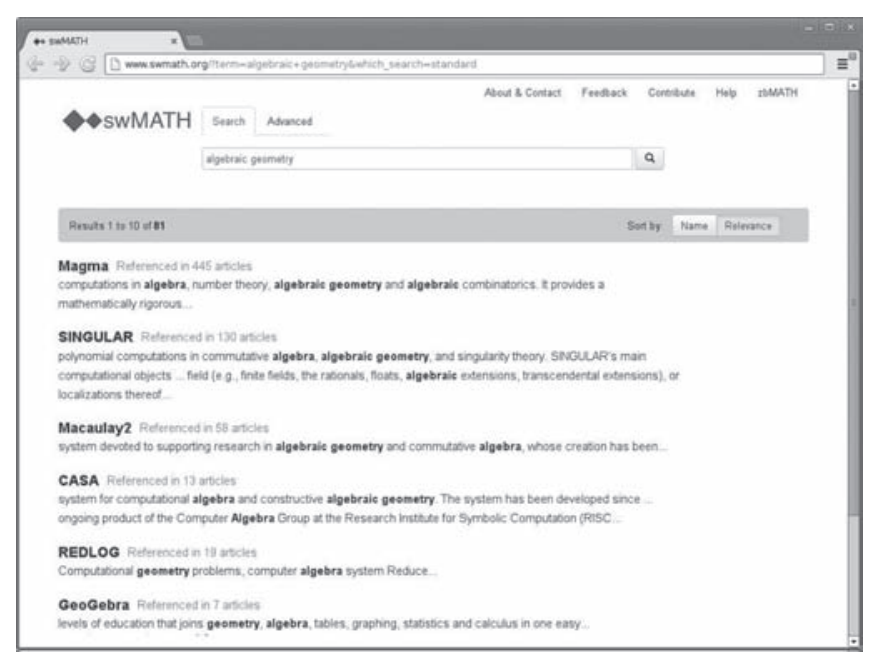

Fig. 2. List view of the search results. 
queries (e.g. search for authors or for certain technical parameters). The search results are displayed in a list view and can be sorted either by name or by relevance (see Figure 2).

The software detail page contains all information available for a certain software package (see Figure 3):

- A short textual description of the software and its areas of application.

- A collection of the most important software metadata (e.g. URL, software authors, licence terms, programming languages, compatible operating systems).

- A collection of the most important keywords associated with the software. ${ }^{10}$

- A collection of references to peer-reviewed articles referring to the software.

- Various filter criteria (e.g. publication year and MSC ${ }^{11}$ code) for restricting the displayed articles to an interesting subset.

- A diagram showing the temporal evolution of publication activities around this software.

- Each reference in the article section is a clickable link leading to the respective entry in Zentralblatt MATH, where additional information on this article can be found (review text, article references, etc.).

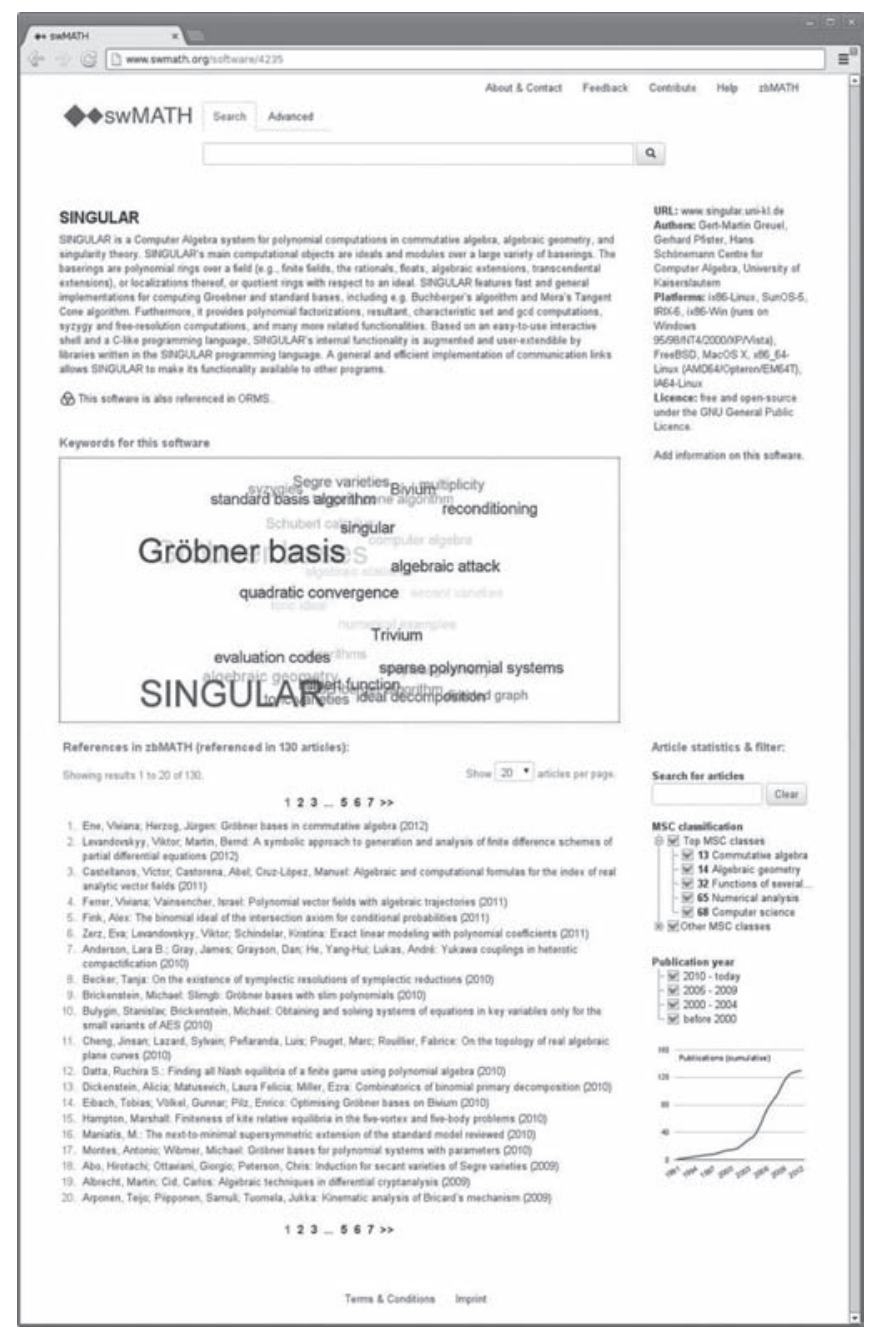

Figure 3. The software detail page.

\section{Feedback and Contributions Welcome!}

Building and maintaining a fairly complete and up-todate database of mathematical software is a very difficult task due to the dynamic character of software development and the heterogeneous quality of the available metadata. We therefore ask everybody with an interest in mathematical software to test and evaluate the swMATH prototype and to provide us with their feedback! Any hints that help us to improve our service are highly appreciated. There are several ways for you to communicate with us: an online feedback form ${ }^{12}$ for general suggestions and a contribution form ${ }^{13}$ for software authors who would like to point us to their software, as well as a contact email address for questions and suggestions of any kind. ${ }^{14}$

\section{swMATH and Other Information Services}

Clearly, swMATH is not the only information service for mathematical software. There are several online portals which contain particular information on software for a certain mathematical research area. In contrast, swMATH aims to be the first software information service which covers all mathematical areas and, in addition, reveals interconnections between different mathematical and applied fields by systematically linking software with relevant publications.

A different strategy is followed in the ORMS ${ }^{15}$ project ("Oberwolfach References on Mathematical Software"), which is carried out by the Mathematisches Forschungsinstitut Oberwolfach. ORMS contains a limited number of high-quality software packages which have been manually selected in cooperation with an international advisory board. Links from swMATH packages to ORMS and back are provided.

\section{Further Schedule}

The official release of the swMATH service is scheduled for December 2013. Until then, a few tasks have to be accomplished: Further improvement of the data quality; completion of the web interface; implementation of update strategies.

The last point is crucial for the long-term perspective of any software service. Software tends to undergo many changes during its development process and information about software quickly becomes out-dated. Therefore, we are working on the implementation of tools (e.g. automatic homepage verifier) and processes to keep swMATH up-to-date and to assure a consistently high data quality. In this respect, swMATH benefits from being embedded in the technical, logistical and personal infrastructure of FIZ Karlsruhe. Recently,

\footnotetext{
${ }^{10}$ The software keywords are automatically generated from the keywords of the articles associated with this software.

${ }^{11}$ MSC = Mathematics Subject Classification, see http://www. zentralblatt-math.org $/ \mathrm{msc} /$.

$12 \mathrm{http}: / /$ www.swmath.org/feedback.

${ }^{13} \mathrm{http} / / /$ www.swmath.org/contribute.

14 contact@swmath.org.

$15 \mathrm{http}$ ://orms.mfo.de.
} 
a process has been established which ensures the detection of new mathematical software packages by the editors of zbMATH.

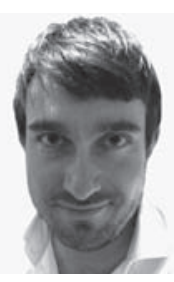

Sebastian Bönisch [sebastian@zentralblattmath.org] studied mathematics and computer science in Heidelberg and got a PhD in mathematics in 2006. He worked as a financial risk analyst and as an IT project leader before joining FIZ Karlsruhe in 2011. He is mainly responsible for the implementation of the swMATH web application.
Gert-Martin Greuel has already been introduced on page 7 as a new member of the Executive Committee of the EMS.

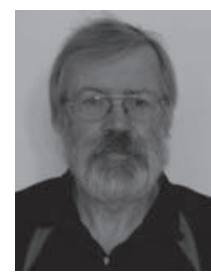

Wolfram Sperber [wolfram. sperber@fizkarlsruhe.de] is working on the editorial staff of Zentralblatt MATH. He has been engaged in diverse projects in the field of specialised information for many years; one of them is the swMATH project. He is involved in the development of the concept of this new service.

\section{Book Reviews}
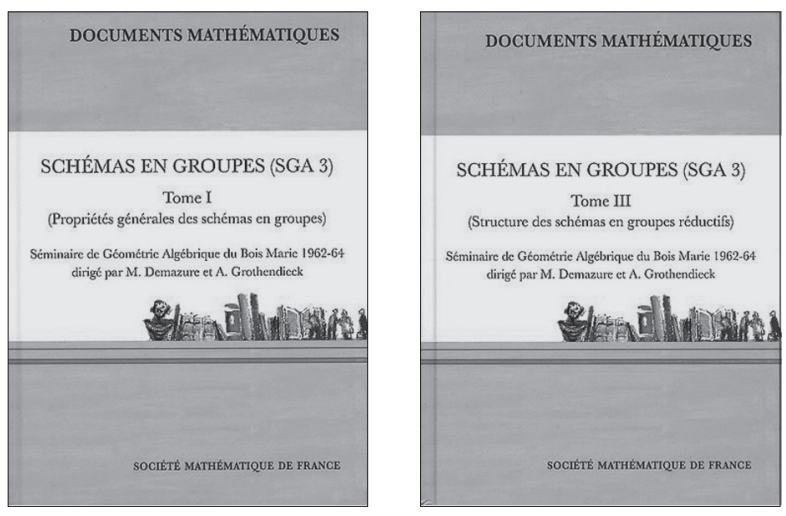

Demazure, Michel (ed.); Grothendieck, Alexander (ed.).

\section{Séminaire de géométrie algébrique du Bois Marie 1962-64. Schémas en groupes (SGA 3).}

Directed by M. Demazure and A. Grothendieck with the collaboration of M. Artin, J.-E. Bertin, P. Gabriel, M. Raynaud and J.-P. Serre.

Tome I: Propriétés générales des schémas en groupes. New annotated edition of the 1970 original published by Springer (French). Documents Mathématiques (Paris) 7. Paris: Société Mathématique de France (ISBN 978-2-85629-323-2/hbk). xxviii, 610 p. (2011).

Tome III: Structure des schémas en groupes réductifs. New annotated edition of the 1970 original published by Springer (French). Documents Mathématiques (Paris) 8. Paris: Société Mathématique de France (ISBN 978-2-85629-324-9/hbk). Iv, 337 p. (2011).

Reviewer: James Milne

The Newsletter thanks Zentralblatt MATH and James Milne for the permission to republish this review, originally appearing as Zbl 1241.14002.
The study of algebraic groups, regarded as groups of matrices, is almost as old as group theory itself - the classical algebraic groups (special linear, orthogonal, symplectic) over the finite prime fields were introduced by Jordan in the 1860s. However, it was not until the work of Maurer, Kolchin and Chevalley that the study of algebraic groups became a subject in its own right. While Kolchin's interest in algebraic groups was as preparation for his study of differential algebraic groups, Chevalley viewed them as a link between Lie groups and finite groups.

A central problem in the subject is the classification of the simple algebraic groups. The similar problem for Lie groups was solved by Killing and Cartan: the classification of simple complex Lie groups is the same as that of simple complex Lie algebras, and Killing and Cartan showed that, in addition to the classical simple Lie algebras, there are only five exceptional algebras. As all semisimple complex Lie groups are algebraic, the classification of simple algebraic groups over $\mathbb{C}$ is the same as that of the simple Lie algebras. This solves the classification problem over $\mathbb{C}$. About 1955, Borel proved his fixed point theorem and thereby obtained his important results on the solvable subgroups of algebraic groups. This enabled Chevalley (in 1956) to extend some of his earlier work and prove that the classification of simple algebraic groups over an algebraically closed field is independent of the field. For fields of nonzero characteristic, this was surprising because the similar statement for Lie algebras is false. Chevalley went further and showed that for split groups, i.e. those containing a split maximal torus, the classification is independent of the base field, algebraically closed or not, and even applies over $\mathbb{Z}$. In his 1965 thesis, Grothendieck's student Demazure showed that Chevalley's classification theory extends in an entirely satisfactory way to split reductive group schemes over arbitrary base schemes. Thus, in a single remarkable decade, the subject of algebraic groups had gone from one in which many of its main results were known only for algebraic groups over $\mathrm{C}$ to one that had achieved a certain maturity as the study of group schemes over arbitrary bases.

Most of this work is documented in the published notes of seminars in the Paris region. The first of these is Séminaire "Sophus Lie" (1954-56), organised by Cartier, which developed (in improved form) the Killing-Cartan 
theory of real and complex Lie algebras. The second is Séminaire Chevalley (1956-58), organised by Chevalley, which explained Borel's work on solvable subgroups and his own work on the classification of simple algebraic groups over algebraically closed fields. Chevalley sketched the extension of his theory to split groups over arbitrary fields (and even $\mathbb{Z}$ ) in a 1961 Bourbaki seminar. Finally, in 1962-64 Grothendieck and Demazure organised the seminar on group schemes that is now referred to as SGA 3. The first two-thirds of the seminar develops the theory of group schemes over an arbitrary base scheme and the final third is a detailed exposition by Demazure of his results on reductive group schemes over an arbitrary base scheme. Many of the participants of these seminars were also involved in the writing of Bourbaki's "Groupes et Algèbres de Lie".

Grothendieck envisaged that his seminars would provide only a first exposition of a topic which would soon be superseded by the "canonical" exposition in EGA (Eléments de Géométrie Algébrique by J. Dieudonné and A. Grothendieck). However, they have proved much more durable than expected - for example, SGA 1 is still widely read and has recently been reprinted in a corrected TEXed version, even though its material has been incorporated into EGA. SGA 3 was never incorporated into EGA - it was to have been Chapter VII - and has been the standard reference on group schemes since the notes for it first became available almost 50 years ago. It has remained the only comprehensive treatment of group schemes over an arbitrary base scheme.

The notes for SGA 3 were originally distributed by IHES (Institut des Hautes Études Scientifiques) in typed mimeographed form under the title SGAD (the reviewer's copy occupies $21 \mathrm{~cm}$ on his bookcase). In 1970, they were reprinted as three volumes in Springer's series Lecture Notes in Mathematics [2]. That version is identical to the original except that some misprints were fixed, part of Exposé VI was rewritten and indexes and tables of contents were added. The present version has been thoroughly revised by the editors Philippe Gille and Patrick Polo, with the support of the mathematical community, especially Demazure, Gabber and Raynaud. Only the first and third volumes are currently available; the remaining volume is expected to become available in 2013.

In the new version, the editors have retained the structure and the numbering of the original but there have been a large number of improvements, which are listed here.

1. The typed originals have been TEXed. This has allowed the editors to improve the typography by replacing underlined letters with calligraphic letters and doubly underlined letters with boldface letters. The residue field at a point $x$ of a scheme is now denoted $\kappa(x)$ and the roots of a semisimple group are denoted $\alpha, \beta, \gamma, \ldots$ rather than $r, s, t, \ldots$. Terminology has been modernised: for example "prescheme/scheme" has been replaced by "scheme/separated scheme".

2. There are numerous small improvements to the text, all carefully footnoted - in Volume 1 there are over
800 of these and in Volume 3 there are about 300. Some of these correct errors in the original and some add comments and references but most expand the original exposition. For example, the editors may add lemmas to make explicit what was only implicit, add arguments omitted in the original or add necessary background material. At the end of each section they have added a bibliography of the additional references cited.

3. In Exposé I, the editors added an eight-page section (Section 6) on $G$-equivariant objects and morphisms in a category, where $G$ is a group object in the category of contravariant set-valued functors on the category.

4. In Exposé III, the editors greatly expanded the zeroth section, which mainly reviews parts of SGA 1 (now in EGA IV [3]). They add several pages (pp.148-152) on complete intersections, which enables them to give a complete proof (instead of a sketch) that a subgroup $H$ of a smooth group scheme $G$ is a local complete intersection in $G$ if it is flat and locally of finite presentation over the base scheme (Prop. 4.15).

5. In Exposé $\mathrm{V}$, the editors have re-ordered the pages, which were shuffled between SGAD and SGA 3(!). The main results in this section concern the construction of quotients by groupoid schemes. The editors have added an eight-page section in which they work out in detail the consequences of these results for quotients by group schemes. They state without proof the more recent results on quotients (Keel, Mori, Kollar).

6. In Exposé VI, the editors have added two sections $(2.6,6)$ explaining results from the 1975 thesis of D. Perrin, which extend some statements for affine group schemes to quasi-compact group schemes. For example, every quasi-compact group scheme over a field is a projective limit of its algebraic quotients.

7. In Exposé VI, the main theorem of Section 5 states that a group scheme and its homogeneous spaces are separated under certain hypotheses. Counterexamples of Gabber show that the original hypotheses are inadequate. The editors have corrected the statement of the theorem and rewritten the section to include a correct proof. Section 11, on affine group schemes and the affine envelope of a general group scheme, has been significantly rewritten and the final two sections $(12,13)$ have been added by the editors.

8. In Exposé VII, several subsections have been enlarged and one has been added.

9. To Volume 3, the editors have added the published version of Demazure's thesis, which summarises the material in the volume and serves as an introduction to it.

The volumes have been handsomely printed. The reviewer can attest that this version is much more pleasant to read, and work with, than the earlier versions. The editors and the Société Mathématique de France are to be congratulated for their efforts in rejuvenating this classic work. Everyone with an interest in group schemes will wish for a copy. 


\section{List of references}

1. J. Dieudonné and A. Grothendieck, Éléments de Géométrie Algébrique. I. Die Grundlehren der mathematischen Wissenschaften 166. Berlin-Heidelberg-New York: Springer-Verlag (1971).

2. M. Demazure and A. Grothendieck, Schémas en groupes. I, II, III. Lecture Notes in Mathematics 151, 152, 153. Berlin-HeidelbergNew York: Springer-Verlag (1970)

3. J. Dieudonné and A. Grothendieck, "Éléments de Géométrie Algébrique. IV. Étude locale des schémas et des morphismes de schémas", Publ. Math., Inst. Hautes Étud. Sci. 20, 101-355 (1964).

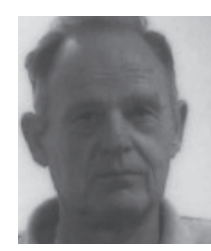

J. S. Milne received his $P h D$ from Harvard University in 1967 under the supervision of John Tate. He was a lecturer at University College London from 1967 until 1969, when he moved to the University of Michigan, where, since 2000, he has been Professor Emeritus.

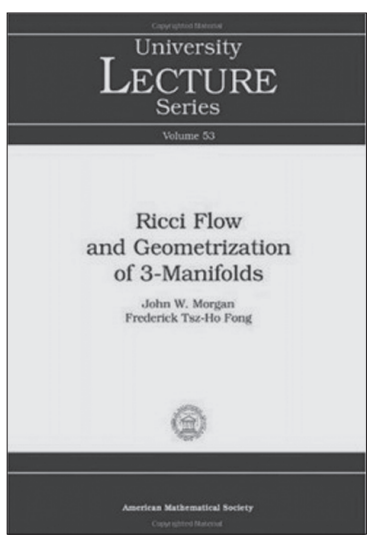

John Morgan

Frederick Fong

Ricci Flow and Geometrization of 3-manifolds

AMS, 2010

University Lecture Series

150 pages

ISBN: 978-0-8218-4963-7

Reviewer: André Neves

Ricci Flow and Geometrization of 3-manifolds, by John Morgan and Frederick Fong, consists of a detailed exposition of one of the greatest achievements in modern mathematics: the proof of the Geometrization Conjecture by Perelman, using Hamilton's Ricci flow.

The intended audience are those interested in knowing more details of the proof without (or before) going through any of the available complete (and long) expositions of Perelman's work on Ricci flow. In the current literature of the proof of the Geometrization Conjecture there are either surveys (30-50 pages) or complete manuscripts (all more than 300 pages and highly technical) and thus the necessity for a book which closes this gap.

The history of the Poincaré Conjecture is well documented and so this will only be covered briefly here to put this book into context.

Ricci flow was introduced by Hamilton in 1982 as a way to deform any metric on a 3-manifold into one which is well understood. He applied it with spectacular success to show that any metric with positive Ricci curvature can be deformed into one which is Einstein and hence of constant sectional curvature. After such a promising first result, Hamilton started to study what would happen if no assumption was made on the initial metric, in which case the flow will develop singularities before the metric becomes round. Hamilton studied this problem in great depth and he developed a programme where, broadly speaking, the idea would be to show that singularities happen only if small cylinders collapse one of its spherical sections, if there is a sphere which col-

lapses, or if both things happen at the same time. If true, one would then be able to remove the "bad" parts of the manifold at the singular time, continue the flow on the new manifold, and repeat this process until the manifold either disappears or converges to something well understood. The initial manifold would then be expressed as connected sums of well known pieces and this suffices to prove the Poincaré Conjecture and the Geometrization Conjecture.

Pinning down the formation of singularities to be $e x$ actly the simple types explained above remained an extremely hard open problem when in 2002 and 2003 Perelman brought fresh and highly original ideas into the field and proved the Poincaré Conjecture and the Geometrization Conjecture, following the approach of Hamilton's programme.

Perelman's ideas were new to the field and so it took some years for the mathematical community to be fully convinced of his arguments. In 2006 three complete expositions of Perelman's work were available: KleninerLott, Cao-Zhu and Morgan-Tian.

The book consists of the lecture notes written during a one semester course, taught by John Morgan, on Perelman's proof of the Geometrization Conjecture. There are six parts to the book, each consisting of around five lectures. Each lecture is written very carefully and expanded so that it can be read by someone who did not attend the course. The writing style is extremely engaging and easy to read. At the beginning of each part there is a short summary of what has been done so far and what the goal is for the next five or so lectures. This helps in making sure the reader never loses sight of the bigger picture.

In the first part, the Geometrization Conjecture is stated, Ricci flow is introduced and the general approach of Perelman's proof is described.

In the second part, the serious work starts. A crucial ingredient of Perelman's work is shown, i.e. the proof of a non-collapsing result. Roughly speaking, it states that along the flow the volume does not collapse at the scale of curvature and this property is essential in order to understand the flow near a singularity. This is done via Lfunctions, introduced by Perelman, which enjoy beautiful monotonicity formulas under the flow and are probably his most amazing contribution. The exposition is selfcontained and everything is carefully explained but a priori knowledge on Ricci flow will surely help the reader being comfortable with this part. 
In the third part, one studies the solutions which arise as blow-ups of singularities (called ?-solutions). An early result of Hamilton-Ivey says that these solutions to Ricci flow have non-negative curvature operator and from the results in the first part we know they are non-collapsed. The bulk of the work goes into classifying the blow-down of ?-solutions: they are shrinking cylinders, shrinking spheres and their quotients. The last lecture in this part is especially pleasant because it summarises what has been done in the previous six and gathers together all the relevant qualitative behaviour of ?-solutions.

In the fourth part, it is shown that every region of high curvature of the flow approaches, after scaling, a ?-solution and so, from the work on the third part, we now have local models for the regions of high curvature. This part is slightly technical because every point of high curvature is studied, not only those where the curvature is a local maximum. The proof is carefully explained and broken into various steps. In the last lecture there is again a nice, concise summary of the proof of the canonical neighbourhood theorem covered in the previous two lectures.

In the fifth part, surgery is performed along the flow whenever a singularity is found. It is shown that at singular time the regions of very large curvature are spheres, balls, cylinders or quotients of these. These regions are removed, caps are attached and the flow is restarted on the new manifold. It is shown that this procedure is done only a finite amount of times in each finite time interval. The overall strategy is very clear but this part is a bit technical because there are many constants one needs to keep track of when doing surgery. Nonetheless, the read- er will surely appreciate that all steps are motivated and that there are constant reminders of work done so far. For instance, in Lecture 25 the main features of surgery are summarised.

In the sixth and final part, the proof of the Geometrization Conjecture is completed. The case to understand is what happens if the flow with surgery never becomes extinct and exists for all time. In this setting we have a thick-thin decomposition of the manifold, where the thick part converges, after rescaling, to a hyperbolic metric and the thin part, which joins the thick part along an incompressible 2-torus, is a graph manifold and thus well understood. This part is again well written and at the beginning there is a recap of the necessary work from the previous parts. The arguments are sketched with the necessary amount of detail to be convincing.

In summary, these are very well written lecture notes which should be extremely useful to those who want to have a detailed idea of Perelman's proof of the Geometrization Conjecture. The authors have made a serious effort so that, despite the technical nature of the subject, the main ideas never get obfuscated and the overall picture is always clear.

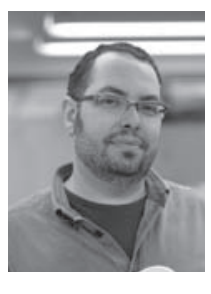

André Neves [aneves@imperial.ac.uk] is a Reader at Imperial College London. He obtained his PhD from Stanford University in 2005. He was an instructor, and later an assistant professor, at Princeton University. He is interested in geometry and analysis.

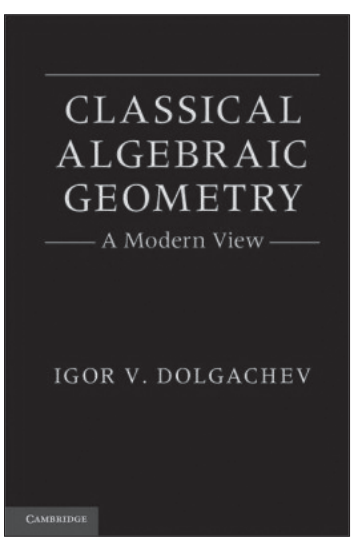

Dolgachev, Igor V.

\section{Classical Algebraic} Geometry. A Modern View

Cambridge: Cambridge

University Press

xii, $639 \mathrm{p}$.

ISBN 978-1-107-01765-8

Reviewer: Werner Kleinert

The Newsletter thanks Zentralblatt MATH and Werner Kleinert for the permission to republish this review, originally appearing as Zbl 1252.14001.

Algebraic geometry has a long and eventful history, in the course of which the subject has undergone several re-formations of its foundations, both conceptually and methodologically. If modern algebraic geometry is understood as the period that began in the second half of the 20th century (when its blending with commutative algebra, homological algebra, category theory, sheaf theory and arithmetic formed its new shape in the hands of A. Grothendieck, K. Kodaira, D. Mumford, J.P. Serre and many others), classical algebraic geometry should be seen as the rich theory developed in the 19th century and early 20th century, especially by the famous schools of algebraic geometry in Italy, Germany, France, Britain and North America. However, many of the great achievements of past generations of algebraic geometers have been generalised in the framework of modern algebraic geometry and have seemingly become oldfashioned (or even obsolete) in our times. As a result, much of the research in classical algebraic geometry only exists in its original form, that is, in a mathematical language incomprehensible to most contemporary scholars in the field.

With a view toward this fact, the main purpose of this monograph is to present some of the most beautiful topics in classical algebraic geometry in a modern context, thereby saving them from sinking into oblivion, on the one hand, and making them accessible to younger generations of algebraic geometers, on the other. 
Actually, the book grew out of the author's lecture notes on the subject, which were (and still are) available from his website at: http://www.math.Isa.umich. $\mathrm{edu} /$ idolga/, along with various other interesting notes.

According to its aim, the book is definitely not a textbook, as it is not meant to provide an introduction to algebraic geometry. Instead, the author's idea was to reconstruct various classical results by using modern techniques, without necessarily explaining their (often ingenious but obscure) original, purely geometrical proofs. Also, as the author points out in the preface, his intention was to present a compendium of material that either cannot be found, is too dispersed to be found easily or is simply not treated adequately in the contemporary research literature. Generally, a profound background knowledge of basic modern algebraic geometry is required and assumed, perhaps as based on the standard texts by I. R. Shafarevich, R. Hartshorne and others.

As for the contents, the book consists of ten chapters, each of which is divided into several sections, including a list of related exercises and some relevant historical notes.

Chapter 1 deals with the classical topic of polarity for projective hypersurfaces with particular emphasis on dual hypersurfaces, polar s-hedra and dual homogeneous forms. The classical works of J. Steiner, J. Plücker, J. Sylvester, G. Salmon, J. Rosanes and other geometers of the 19th century are carefully analysed in this chapter.

Chapter 2 studies conics and quadric surfaces in further detail, especially with a view toward their polarity properties and their allied duality theory. Chapter 3 turns to the classical theory of plane cubic curves, with a focus on their Hessians, their polars, their projective generation and their invariant theory. Determinantal equations are discussed in Chapter 4, where a modern approach to determinantal plane curves and their moduli, a treatment of determinantal hypersurfaces and linear determinantal representations of surfaces via arithmetically Cohen-Macaulay sheaves play a central role. Chapter 5 is devoted to theta characteristics of algebraic curves, including related topics such as theta functions, quadratic forms and their Arf invariants hyperelliptic curves, Jacobians, Steiner complexes, the Scorza correspondence for curves and contact hyperplanes of canonical curves.

Chapter 6 analyses plane quartics, their bitangents, their determinant equations, their even theta characteristics, their particular invariant theory and their automorphisms. A wealth of classic results from the 19th century are illuminated in this chapter, together with many references to modern developments in the research on plane quartics.

Cremona transformations are the subject of study in Chapter 7, where the related theory of birational maps between algebraic surfaces is also introduced, in its modern, sheaf-theoretic and cohomological setting. Chapter 8 takes up another classical topic, namely the study of del Pezzo surfaces (or Fano surfaces). The account given here is particularly comprehensive and detailed, with many subtle and refined results concerning del Pezzo surfaces of low degree.
The rich theory of cubic surfaces is reviewed in Chapter 9, with the central topics being the lines on a nonsingular cubic in $\mathrm{P}^{3}$, Schur's quadrics, singularities of cubic surfaces, determinantal equations of normal cubic surfaces, moduli of cubic surfaces, automorphisms of cubic surfaces and various special cubic surfaces. Finally, Chapter 10 is titled "Geometry of lines". In this concluding part of the book, the author explains Grassmannians and Schubert varieties, secant varieties of Grassmannians of lines, linear line complexes in Grassmannians of lines and their applications, linear systems of linear line complexes, quadratic line complexes, the geometry of the intersection of two quadrics, Kummer surfaces, harmonic line complexes, the geometry of ruled surfaces, the Cayley-Zeuthen formulas and numerous concrete examples. All this classical material is instructively presented in a modern context, thereby effectively combining beautiful classical ideas with powerful contemporary techniques in algebraic geometry.

As mentioned before, each chapter comes with its own list of related exercises. Most of these problems are rather challenging and of a theoretical nature. Nevertheless, they provide a wealth of additional results and examples in quite a unique manner and are an invitation to active research. Just as useful are the historical notes at the end of each chapter, which also provide invaluable hints to the classical research literature. The bibliography contains more than 600 precise references, which surely represents another great feature of this masterful treatise.

All together, the author has rendered a great, truly invaluable service to the community of algebraic geometers worldwide. No doubt, this great book is a product of ultimate enthusiasm, ethical principles and expertise, which will help preserve the precious legacy of classical algebraic geometry for further generations of researchers, teachers and students in the field.

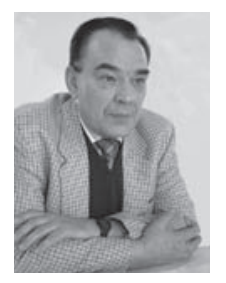

Werner Kleinert received his doctoral degree in commutative algebra in 1971. After his postdoctoral qualification (habilitation) in the field of algebraic geometry in 1979, he was promoted to university lecturer at Humboldt University in Berlin, an academic position that he held there until his retirement in 2010. His main research interests have always been the geometry of moduli spaces of algebraic curves and abelian varieties, together with related topics such as Riemann surfaces, theta functions and Teichmueller theory. 


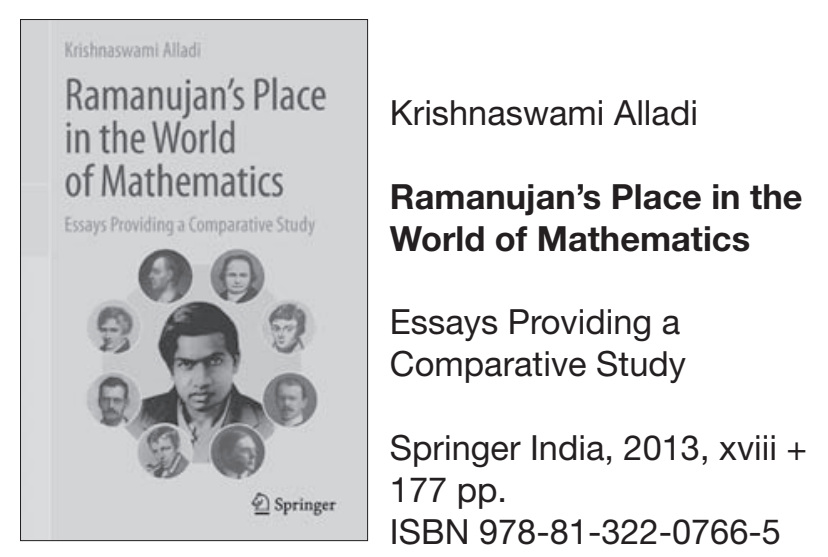

Reviewer: Themistocles M. Rassias

Srinivasa Ramanujan (1887-1920) is one of the greatest mathematicians in history. What makes Ramanujan unique is the manner in which he obtained his results and the spectacular beauty and depth of his discoveries. Born into a poor Hindu Brahmin family in rural South India, Ramanujan had no formal education. He startled the British mathematician Hardy by sending him several bewildering formulae in a series of letters. There is a legend that the Hindu Goddess Namagiri would come in Ramanujan's dreams and give him these formulae, which he recorded in two notebooks. On seeing the letters, Hardy was convinced that Ramanujan was on a par with Euler and Jacobi in sheer manipulative ability and so he invited the Indian genius to work with him at Cambridge University. The rest is history. In a short span of five years, Ramanujan published several fundamental papers by himself and with Hardy that revolutionised many areas of mathematics and revealed surprising connections between fields apparently unrelated. For his groundbreaking work he was elected Fellow of the Royal Society and Fellow of Trinity College, Cambridge, even though he did not possess a college degree! Unfortunately, the rigours of life in England during World War I, and his own peculiar habits, took a toll on his health. Ramanujan returned to India in 1919 a very sick man, and died within a year. But what he has left behind in his notebooks and papers have engaged mathematicians ever since and will continue to be of influence in the years ahead. His work has had a profound and deep impact in a wide range of areas both within and outside of mathematics.

One of the best ways to understand Ramanujan and his mathematics is to study his life and work in comparison to other outstanding mathematicians in history whose lives and works have things in common with Ramanujan. Some of them, like Ramanujan, underwent great difficulties in life but, undeterred by these obstacles, produced work of the highest quality. After providing an evaluation of Ramanujan and discussing the possible impact of his work in the years following his centenary, there is a collection of essays on 13 mathematical luminaries that provides a comparative study. These essays are really the heart of the book.

Much has been written about Ramanujan's life and work and justifiably so. But in the words of George An- drews (one of the foremost authorities on Ramanujan's work) who has written the Foreword: "This, however, is the first [book] to provide what might be termed as a perspective overview." Following these comparative essays are articles describing various aspects of Ramanujan's work, such as his remarkable discoveries on partitions, his startling identities for the number $\pi$ and his fundamental work on quadratic forms. Also included in the book are reviews of four important books on Ramanujan written since the Ramanujan Centennial. There is even a review of the play "Partition" on Ramanujan that was produced in 2005. Finally the book concludes with articles on what is being done now to preserve and foster the legacy of Ramanujan, such as the launch of the Ramanujan Journal and the creation of the SASTRA Ramanujan Prize, as well as the author's efforts in such enterprises.

All these articles were written by the author since the Ramanujan Centennial in 1987 and appeared mostly in the Hindu, India's National Newspaper, for the benefit of the general public, with others in journals like the American Scientist and publications of the Mathematical Association of America. They have been brought out collectively in the form of a book on what would have been Ramanujan's 125th birthday (22 December 2012).

As Andrews writes in his Foreword: "Alladi, who has worked in several areas of number theory and analysis, and who, as the editor of The Ramanujan Journal, is uniquely qualified to write these historical sketches which provide an unusual and compelling view of Ramanujan." The book is an enlightening study of Ramanujan as a mathematician and as a human being and will appeal to mathematicians, students and the general public interested in mathematics.

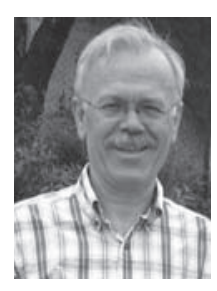

Themistocles M. Rassias [trassias@math. ntua.gr] is a professor of mathematics at the National Technical University, Athens, Greece. He received his PhD under the supervision of Professor Steven Smale at the University of California at Berkeley. He is a well-known author of many articles and books, mainly in the areas of mathematical analysis, global analysis, geometry and topology. He is a member of the editorial boards of several international mathematical journals including the EMS Newsletter. 


\section{Solved}

\section{and Unsolved} Problems

Themistocles M. Rassias (National Technical University of Athens, Greece)

From the most skilful definition, free as it might be of any inner contradictions, one can never deduce a new fact.

Max Planck (1858-1947), Nobel Prize in Physics, 1918

\section{Six new problems-solutions solicited}

Solutions will appear in a subsequent issue.

107. Find all differentiable functions $f: \mathbb{R} \rightarrow \mathbb{R}$ with continuous derivative $f^{\prime}$ such that the following properties hold:

1. $f\left(x+f^{\prime}(x)\right)=f(x)$ for all $x \in \mathbb{R}$;

2. The derivative $f^{\prime}$ vanishes at a single point.

(Dorin Andrica, Babeş-Bolyai University of Cluj-Napoca, Romania)

108. For a positive integer $n$ denote by $a_{n}$ the number of linear functions defined by $f(x)=a x+b$, where $a, b \in\{1,2, \cdots, n\}$, having an integer root. Prove that

$$
\lim _{n \rightarrow \infty} \frac{a_{n}}{n \ln n}=1
$$

(Dorin Andrica, Babeş-Bolyai University of Cluj-Napoca, Romania)

109. Find all continuous functions $f, g:(0,1) \rightarrow R$ that satisfy the functional inequality

$$
x f(x)+(1-x) g(1-x) \geq x f(y)+(1-x) g(1-y)
$$

for all $x, y \in(0,1)$.

(Prasanna K. Sahoo, Department of Mathematics, University of Louisville, USA)

110. Let $f:(1,+\infty) \rightarrow \mathbb{R}$ be a continuously differentiable function. It is known that the condition $\lim _{x \rightarrow \infty} f^{\prime}(x)=0$ is not sufficient to imply $\lim _{x \rightarrow \infty} f(x)=l \in \mathbb{R}$. But what about if we use another condition of the form $\lim _{x \rightarrow \infty} x^{\alpha} f^{\prime}(x)=0(\alpha>0)$ ?

Find for what values of $\alpha(\alpha>0)$ the following statement is true: If $\lim _{x \rightarrow \infty} x^{\alpha} f^{\prime}(x)=0$ then $\lim _{x \rightarrow \infty} f(x)=l \in \mathbb{R}$.

(Panagiotis T. Krasopoulos, Athens, Greece)
111. Let $a, b$ be integers such that $2 \leq a<b$. Prove that

$$
\sum_{n=1}^{\infty}\left(\frac{(a-1)(b-1)\left\lfloor n \log _{a} b\right\rfloor}{b^{n}}+\frac{b-1}{a^{\left\lfloor n \log _{a} b\right\rfloor}}-\frac{1}{a^{\left\lfloor\log _{a} n\right\rfloor} b^{\left\lfloor\log _{b} n\right\rfloor}}\right)=1,
$$

where $\lfloor x\rfloor$ denotes the largest integer not greater than $x$.

(Carlo Sanna, Università degli studi di Torino, Turin, Italy)

112. Let $\alpha>0$ be a real number. Find the value of

$$
\lim _{n \rightarrow \infty} n \int_{-1}^{0}\left(x+\frac{x^{2}}{2}+e^{\alpha \cdot x}\right)^{n} d x
$$

(Ovidiu Furdui, Technical University of Cluj-Napoca, Romania)

\section{Two new open problems}

113*. Find all functions $f:(0,1) \rightarrow R$ that satisfy the functional equation

$$
f(x y)+f(x(1-y))+f(y(1-x))+f((1-x)(1-y))=0
$$

for all $x, y \in(0,1)$.

(Prasanna K.Sahoo, Department of Mathematics, University of Louisville, USA)

114. Find all functions $f:(0,1) \rightarrow R$ that satisfy the functional equation

$$
f(x y)+f((1-x)(1-y))=f(x(1-y))+f(y(1-x))
$$

for all $x, y \in(0,1)$.

(Prasanna K.Sahoo, Department of Mathematics, University of Louisville, USA)

Note: If one assumes any regularity condition such as continuity or measurability then the solutions of these two equations are known.

\section{Solutions}

99. For any positive integer $n$ let $x_{n}$ be the unique positive root of the polynomial

$$
f_{n}(t)=t^{3}+3 t^{2}-\frac{12}{n^{2}}
$$

Compute the limit

$$
\lim _{n \rightarrow \infty} n\left(n x_{n}-2\right) .
$$

(Dorin Andrica, Babes-Bolyai University of Cluj-Napoca, Romania)

Solution by the proposer. Because of the facts $f_{n}(0)=-\frac{12}{n^{2}}<0$, $\lim _{n \rightarrow \infty} f_{n}(t)=+\infty$ and

$$
f_{n}^{\prime}(t)=3 t^{2}+6 t>0
$$

for every $t>0$, it follows that $f_{n}$ is strictly increasing on $(0, \infty)$ and the equation $f_{n}(t)=0$ has a unique positive root $x_{n}$.

From

$$
x_{n}^{3}+3 x_{n}^{2}-\frac{12}{n^{2}}=0
$$


it follows that

$$
3 x_{n}^{2}<\frac{12}{n^{2}} .
$$

Hence, we have $x_{n}<\frac{2}{n}$, that is, $0<n x_{n}<2, n=1,2, \cdots$.

Therefore, the sequence $\left(n x_{n}\right)_{n \geq 1}$ is bounded.

Relation (2) is equivalent to

$$
\frac{\left(n x_{n}\right)^{3}}{n}+3\left(n x_{n}\right)^{2}-12=0 .
$$

From $\lim _{n \rightarrow \infty} \frac{\left(n x_{n}\right)^{3}}{n}=0$, it follows that $\lim _{n \rightarrow \infty} n x_{n}=2$.

Relation (3) is equivalent to

$$
\left(n x_{n}\right)^{3}+3 n\left(n x_{n}-2\right)\left(n x_{n}+2\right)=0
$$

and hence

$$
8+12 \lim _{n \rightarrow \infty} n\left(n x_{n}-2\right)=0
$$

Therefore

$$
\lim _{n \rightarrow \infty} n\left(n x_{n}-2\right)=-\frac{2}{3} .
$$

Also solved by Jim Bradley (Devon, UK), Yakar Kannai (Weizmann Institute, Israel), P. T. Krasopoulos (Athens, Greece), Paolo Secchi (University of Brescia, Italy), I. I. Stavroulakis (Lykeion, Dodonea, Ioannina, Greece), Li Zhou (Polk State College, Florida, USA)

100. Let $f:(0,1) \rightarrow \mathbb{R}$ be infinitely differentiable (i.e., $\left.C^{\infty}(0,1)\right)$, with the property that for any $x \in(0,1)$ there exists some $n=n(x)$ nonnegative integer such that $f^{(n)}(x)=0$. Show that $f$ is a polynomial.

(Francesco Sica, University of Waterloo, Canada)

Solution by the proposer. The general idea is to proceed by contradiction and construct a sequence of nested open intervals $\left(a_{n}, b_{n}\right) \subset$ $(0,1)$ such that $f^{(m)}(x) \neq 0$ for $m<n$ and $x \in\left(a_{n}, b_{n}\right)$. If we can ensure that $\cap_{n \geq 0}\left(a_{n}, b_{n}\right) \neq \emptyset$ then at any $x$ in this intersection neither $f$ nor any of its derivatives will vanish, contradicting the assumption.

However, this construction is nontrivial to carry out. If one carves out the zeroes of successive derivatives from $(0,1)$, it is not clear that we don't arrive at the empty set. In fact, the open set where $f(x) \neq 0$ is a disjoint countable union of open intervals but on each of these intervals a derivative could a priori vanish identically. It is the way these intervals are connected which will prevent this.

A crucial role is played by what we will call strong zeroes of derivatives, namely points $x \in(0,1)$ for which there exists $n \geq 0$ such that $f^{(n+k)}(x)=0$ for all $k \geq 0$ (in which case we say that $x$ is a strong zero of $\left.f^{(n)}\right)$. In fact, the set of strong zeroes is dense in $(0,1)$, an immediate consequence of Baire's category theorem but this can be proved by the following more elementary argument, which we single out since it will be used repeatedly in our construction of $\left(a_{n}, b_{n}\right)$.

Fact 1: If a sequence $\left(x_{m}\right)$ of zeroes of some $f^{(n)}$ has an accumulation point $c \in(0,1)$ then this accumulation point is a strong zero of $f^{(n)}$.

This is proved by repeated applications of Rolle's theorem: by extracting a subsequence, we can suppose $\left(x_{m}\right)$ is monotone (say increasing) with limit $c$. Firstly, by continuity of $f^{(n)}$ we have $f^{(n)}(c)=$ 0 . Next, in each interval $\left(x_{m}, x_{m+1}\right)$ there exists a zero $x_{m}^{\prime}$ of $f^{(n+1)}$ by Rolle's theorem. By the squeeze theorem, $\lim x_{m}^{\prime}=c$ and therefore $f^{(n+1)}(c)=0$. Continuing to apply Rolle's theorem we find interleaved sequences of zeroes of consecutive derivatives, all tending to $c$, and therefore the fact is proved.
Fact 2: Any interval $[a, b] \subset(0,1)$ contains a strong zero of some derivative of $f$. We argue by contradiction to prove this: otherwise, by Fact 1 , each $f^{(n)}$ has only a finite number of zeroes in $[a, b]$ since it is a compact set. Since there are countably many derivatives, this implies that the total number of zeroes of derivatives in $[a, b]$ is at most countable, contradicting the hypothesis.

Fact 3: Let $\left(a_{n}, b_{n}\right)$ be a sequence of nested intervals satisfying $a_{n}<a_{n+1}$ and $b_{n}>b_{n+1}$ for all $n \geq 0$, in which case we will say that $\left(a_{n+1}, b_{n+1}\right)$ is strictly nested in $\left(a_{n}, b_{n}\right)$. Then $\cap_{n \geq 0}\left(a_{n}, b_{n}\right) \neq \emptyset$.

Indeed, in this case $\cap_{n \geq 0}\left(a_{n}, b_{n}\right) \supset \cap_{n \geq 0}\left[a_{n}, b_{n}\right] \neq \emptyset$ by a wellknown argument.

We are now ready to introduce our construction. We will define a sequence $\left(n_{m}\right)$ of strictly increasing integers, together with a sequence of strictly nested intervals $\left(a_{m}, b_{m}\right) \subset(0,1)$ on which $f^{(n)}$ has no zeroes for $n<n_{m}$.

Let

$n_{0}=\min \left\{n \in \mathbb{N} \cup\{0\}\right.$ such that $f^{(n)}$ has a strong zero in $\left.(0,1)\right\}$,

which exists by Fact 2 . Note that by Fact 1 , for any $[a, b] \subset(0,1)$, there are finitely many zeroes of $f^{(n)}$ for $n<n_{0}$. If $f^{\left(n_{0}\right)} \equiv 0$ on $(0,1)$ then $f$ is a polynomial and we are done and there is nothing more to show. Otherwise, we let $\left(\tilde{a}_{0}, \tilde{b}_{0}\right)$ be an interval strictly nested in $(0,1)$ where $f^{\left(n_{0}\right)} \neq 0$. Let $x_{0} \in(0,1)$ be a strong zero of $f^{\left(n_{0}\right)}$. We suppose, without loss of generality, that $x_{0} \leq \tilde{a}_{0}$ (otherwise, replace $<$ by $>$ and sup by inf in the following, or equivalently replace $f(x)$ by $f(1-x)$ ). Define

$$
a_{0}=\sup \left\{x<\tilde{b}_{0} \text { such that } x \text { is a strong zero of } f^{\left(n_{0}\right)}\right\}
$$

and note that $0<a_{0} \leq \tilde{a}_{0}$ and that $a_{0}$ is a strong zero of $f^{\left(n_{0}\right)}$. Since $\left(a_{0}, \tilde{b}_{0}\right)$ is strictly nested in $(0,1)$, by the above there are finitely many zeroes of all $f^{(n)}$ with $n<n_{0}$. We can therefore choose $a_{0}<b_{0}<\tilde{b}_{0}$ such that $\left(a_{0}, b_{0}\right)$ contains no zero of $f^{(n)}$ for $n<n_{0}$. The interval $\left(a_{0}, b_{0}\right)$ satisfies the following three properties $\left(\mathrm{A}_{m}\right),\left(\mathrm{B}_{m}\right)$ and $\left(\mathrm{C}_{m}\right)$ for $m=0$.

$\left(\mathrm{A}_{m}\right)$ The interval $\left(a_{m}, b_{m}\right)$ does not contain zeroes of $f^{(n)}$ for any $n<n_{m}$.

$\left(\mathrm{B}_{m}\right)$ The zeroes of $f^{\left(n_{m}\right)}$ in $\left(a_{m}, b_{m}\right)$ are not strong zeroes and do not accumulate inside $\left(a_{m}, b_{m}\right)$.

$\left(\mathrm{C}_{m}\right)$ For any $k \geq 0$, we have $f^{\left(n_{m}+k\right)} \not \equiv 0$ in $\left(a_{m}, b_{m}\right)$.

By construction $\left(a_{0}, b_{0}\right)$ satisfies $\left(\mathrm{A}_{0}\right)$. Let us show $\left(\mathrm{B}_{0}\right)$ and $\left(\mathrm{C}_{0}\right)$. By definition of $a_{0}$, and since $b_{0}<\tilde{b}_{0}$, the interval $\left(a_{0}, b_{0}\right)$ does not contain strong zeroes of $f^{\left(n_{0}\right)}$. Therefore, by Fact $1,\left(\mathrm{~B}_{0}\right)$ holds. To show $\left(\mathrm{C}_{0}\right)$, we recall that, by construction, one of the endpoints of $\left(a_{0}, b_{0}\right)$ (in this case $a_{0}$, since we supposed $x_{0}<\tilde{a}_{0}$ ) will be a strong zero of $f^{\left(n_{0}\right)}$. If $f^{\left(n_{0}+k\right)} \equiv 0$ in $\left(a_{0}, b_{0}\right)$ for $k \geq 1$ then $f^{\left(n_{0}+k-1\right)} \equiv$ constant in $\left(a_{0}, b_{0}\right)$ and this constant must be 0 since $f^{\left(n_{0}+k-1\right)}\left(a_{0}\right)=0$. We can continue this way up to deduce that $f^{\left(n_{0}\right)} \equiv 0$ in $\left(a_{0}, b_{0}\right)$, contradicting $\left(\mathrm{B}_{0}\right)$.

We now show the inductive step of the construction, namely that from $n_{m}$ and $\left(a_{m}, b_{m}\right)$ satisfying $\left(\mathrm{A}_{m}\right),\left(\mathrm{B}_{m}\right)$ and $\left(\mathrm{C}_{m}\right)$ we can construct $n_{m+1}>n_{m}$ and $\left(a_{m+1}, b_{m+1}\right)$ strictly nested in $\left(a_{m}, b_{m}\right)$ which satisfy $\left(\mathrm{A}_{m+1}\right),\left(\mathrm{B}_{m+1}\right)$ and $\left(\mathrm{C}_{m+1}\right)$.

We define

$n_{m+1}=\min \left\{n \in \mathbb{N} \cup\{0\}\right.$ such that $f^{(n)}$ has a strong zero in $\left.\left(a_{m}, b_{m}\right)\right\}$,

which exists by Fact 2 . Note also that by $\left(\mathrm{A}_{m}\right)$ and $\left(\mathrm{B}_{m}\right)$, we have $n_{m+1}>n_{m}$. Also, by Fact 1 , for $n_{m} \leq n<n_{m+1}$, the zeroes of $f^{(n)}$ do not accumulate inside $\left(a_{m}, b_{m}\right)$ (i.e. they are isolated).

By $\left(\mathrm{C}_{m}\right), f^{\left(n_{m+1}\right)} \not \equiv 0$ in $\left(a_{m}, b_{m}\right)$ and we choose $\left(\tilde{a}_{m+1}, \tilde{b}_{m+1}\right)$ strictly nested in $\left(a_{m}, b_{m}\right)$ such that $f^{\left(n_{m+1}\right)}(x) \neq 0$ for all $x \in$ 
$\left(\tilde{a}_{m+1}, \tilde{b}_{m+1}\right)$. Let $x_{m+1} \in\left(a_{m}, b_{m}\right)$ be a strong zero of $f^{\left(n_{m+1}\right)}$ and assume without loss of generality that $\tilde{b}_{m+1} \leq x_{m+1}$. Define

$$
b_{m+1}=\inf \left\{x>\tilde{a}_{m+1} \text { such that } x \text { is a strong zero of } f^{\left(n_{m+1}\right)}\right\} .
$$

Note that $b_{m}>x_{1} \geq b_{m+1} \geq \tilde{b}_{m+1}$ and that $b_{m+1}$ is a strong zero of $f^{\left(n_{m+1}\right)}$. Since $\left(\tilde{a}_{m+1}, b_{m+1}\right)$ is strictly nested in $\left(a_{m}, b_{m}\right)$, it contains only finitely many zeroes of all $f^{(n)}$ with $n_{m} \leq n<n_{m+1}$. Therefore, by choosing an appropriate $\tilde{a}_{m+1}<a_{m+1}<b_{m+1}$, we can ensure that $\left(a_{m+1}, b_{m+1}\right)$ does not contain zeroes of any $f^{(n)}$ with $n_{m} \leq n<n_{m+1}$. Note that $\left(a_{m+1}, b_{m+1}\right)$ is strictly nested in $\left(a_{m}, b_{m}\right)$ and therefore does not contain zeroes of any $f^{(n)}$ with $n<n_{m}$ by $\left(\mathrm{A}_{m}\right)$. Hence $\left(\mathrm{A}_{m+1}\right)$ holds for $\left(a_{m}, b_{m}\right)$ and $n_{m+1}$. Properties $\left(\mathrm{B}_{m+1}\right)$ and $\left(\mathrm{C}_{m+1}\right)$ are proved similarly, as before, thus showing the inductive step of the construction.

Since $\left(n_{m}\right)$ is strictly increasing, we can conclude that $n_{m} \geq m$ and therefore no function $f, f^{\prime}, \ldots, f^{(m-1)}$ has zeroes in $\left(a_{m}, b_{m}\right)$. Since there exists $y \in \cap_{m \geq 0}\left(a_{m}, b_{m}\right)$ by Fact 3 , we have that $f^{(n)}(y) \neq$ 0 for all $n \geq 0$, contradicting the hypothesis and hence $f$ must be a polynomial on $(0,1)$.

101. Examine if a two-variate algebraic polynomial $p(x, y)$ such that $p(x, y) \geq 0$ for all $x, y \in \mathbb{R}$ always possesses a point of local minimum.

(Vladimir Protasov, Moscow State University, Russia)

Solution by the proposer. There is a well-known example of a twovariate polynomial that does not attain its global minimum. This is the polynomial $p(x, y)$ defined by

$$
p(x, y)=x^{2}+(1+x y)^{2} .
$$

It appears that $p(x, y)$ has no local minima either. Indeed

$$
p_{x}^{\prime}(x, y)=2 x+2 y(1+x y) \text { and } p_{y}^{\prime}(x, y)=2 x(1+x y) .
$$

If $p_{y}^{\prime}(x, y)=0$ then either $x=0$ or $1+x y=0$. In the first case the equation $p_{x}^{\prime}(x, y)=0$ implies that $y=0$. In the second case it implies that $x=0$, which is now impossible. Thus, the only point where both partial derivatives vanish is $(x, y)=(0,0)$. However, this is not a local minimum, since

$$
p(t,-t)=1-t^{2}+t^{4}<1=p(0,0)
$$

for all $t \in(-1,1)$.

\section{Also solved by Yakar Kannai (Weizmann Institute, Israel)}

102. Find the value of the series

$$
\sum_{n=1}^{\infty} \frac{1}{n}\left(\frac{1}{n+1}-\frac{1}{n+2}+\frac{1}{n+3}-\cdots\right) .
$$

(Ovidiu Furdui, Technical University of Cluj-Napoca, Romania)

Solution by the proposer. Claim that the value of the series equals

$$
\frac{1}{2} \zeta(2)-\frac{1}{2} \ln ^{2} 2
$$

We have

$$
\begin{aligned}
\frac{1}{n+1}-\frac{1}{n+2}+\frac{1}{n+3}-\cdots & =\int_{0}^{1}\left(x^{n}-x^{n+1}+x^{n+2}-\cdots\right) d x \\
& =\int_{0}^{1} \frac{x^{n}}{1+x} d x
\end{aligned}
$$

It follows that

$$
\begin{aligned}
& \sum_{n=1}^{\infty} \frac{1}{n}\left(\frac{1}{n+1}-\frac{1}{n+2}+\frac{1}{n+3}-\cdots\right)=\sum_{n=1}^{\infty} \frac{1}{n} \int_{0}^{1} \frac{x^{n}}{1+x} d x \\
& =\int_{0}^{1} \frac{1}{1+x}\left(\sum_{n=1}^{\infty} \frac{x^{n}}{n}\right) d x \\
& =-\int_{0}^{1} \frac{\ln (1-x)}{1+x} d x \\
& \stackrel{1-x=y}{=}-\int_{0}^{1} \frac{\ln y}{2-y} d y \\
& =-\int_{0}^{1} \frac{\ln (y / 2)+\ln 2}{2-y} d y \\
& =-\int_{0}^{1} \frac{\ln (y / 2)}{2-y} d y-\ln ^{2} 2 \\
& \stackrel{y / 2=t}{=}-\int_{0}^{1 / 2} \frac{\ln t}{1-t} d t-\ln ^{2} 2 \\
& =-\int_{1 / 2}^{1} \frac{\ln (1-t)}{t} d t-\ln ^{2} 2 \\
& =-\left(\int_{0}^{1}-\int_{0}^{1 / 2}\right) \frac{\ln (1-t)}{t} d t \\
& -\ln ^{2} 2 \\
& =L i_{2}(1)-L i_{2}\left(\frac{1}{2}\right)-\ln ^{2} 2 \\
& =\frac{1}{2}\left(\zeta(2)-\ln ^{2} 2\right)
\end{aligned}
$$

and the problem is solved.

We have used in our calculations the dilogarithm function, which is defined (see [1, Entry 3, p. 106]) by

$$
L i_{2}(z)=\sum_{n=1}^{\infty} \frac{z^{n}}{n^{2}}=-\int_{0}^{z} \frac{\log (1-t)}{t} d t, \quad|z| \leq 1 .
$$

Also,

$$
L i_{2}(1)=\zeta(2)
$$

and an application of Landen's formula (see [1, Entry 10, p. 107])

$$
L i_{2}(z)+L i_{2}(1-z)=\frac{\pi^{2}}{6}-\log z \cdot \log (1-z)
$$

imply that

$$
\operatorname{Li}_{2}\left(\frac{1}{2}\right)=\frac{\pi^{2}}{12}-\frac{\ln ^{2} 2}{2}
$$

\section{References}

[1] H. M. Srivastava and J. Choi, Series Associated with the Zeta and Related Functions, Kluwer Academic Publishers, 2001

Also solved by Jim Bradley (Devon, UK), Paolo Secchi (University of Brescia, Italy), I. I. Stavroulakis (Lykeion, Dodonea, Ioannina, Greece), Li Zhou (Polk State College, Florida, USA)

103. Let $f:[a, b] \rightarrow \mathbb{R}$ be a convex function defined on the closed interval $[a, b]$. Prove that for any $x \in(a, b)$ the following holds

$$
\frac{1}{2}\left[(b-x)^{2} f_{+}^{\prime}(x)-(x-a)^{2} f_{-}^{\prime}(x)\right] \leq \int_{a}^{b} f(t) d t-(b-a) f(x) .
$$

The constant $\frac{1}{2}$ on the left side of (4) is sharp in the sense that it cannot be replaced by a larger real constant.

(Sever S. Dragomir, Victoria University, Australia) 
Solution by the proposer. It is easy to see that for any locally absolutely continuous function $f:(a, b) \rightarrow \mathbb{R}$, we have the identity

$$
\int_{a}^{x}(t-a) f^{\prime}(t) d t+\int_{x}^{b}(t-b) f^{\prime}(t) d t=f(x)-\int_{a}^{b} f(t) d t,
$$

for any $x \in(a, b)$ where $f^{\prime}$ is the derivative of $f$ which exists a.e. on $(a, b)$.

Since $f$ is convex, then it is locally Lipschitzian and thus (5) holds. Moreover, for any $x \in(a, b)$, we have the inequalities

$$
f^{\prime}(t) \leq f_{-}^{\prime}(x) \text { for a.e. } t \in[a, x]
$$

and

$$
f^{\prime}(t) \geq f_{+}^{\prime}(x) \text { for a.e. } t \in[x, b] .
$$

If we multiply (6) by $t-a \geq 0, t \in[a, x]$, and integrate on $[a, x]$, we get

$$
\int_{a}^{x}(t-a) f^{\prime}(t) d t \leq \frac{1}{2}(x-a)^{2} f_{-}^{\prime}(x)
$$

and if we multiply (7) by $b-t \geq 0, t \in[x, b]$, and integrate on $[x, b]$, we also have

$$
\int_{x}^{b}(b-t) f^{\prime}(t) d t \geq \frac{1}{2}(b-x)^{2} f_{+}^{\prime}(x) .
$$

Finally, if we subtract (9) from (8) and use the representation (5) we deduce the desired inequality (4).

Now, assume that (4) holds with a constant $C>0$ instead of $\frac{1}{2}$, that is,

$$
C\left[(b-x)^{2} f_{+}^{\prime}(x)-(x-a)^{2} f_{-}^{\prime}(x)\right] \leq \int_{a}^{b} f(t) d t-(b-a) f(x) .
$$

Consider the convex function defined by

$$
f_{0}(t):=k\left|t-\frac{a+b}{2}\right|, k>0, \quad t \in[a, b] .
$$

Then

$$
f_{0^{+}}^{\prime}\left(\frac{a+b}{2}\right)=k, \quad f_{0^{-}}^{\prime}\left(\frac{a+b}{2}\right)=-k, \quad f_{0}\left(\frac{a+b}{2}\right)=0
$$

and

$$
\int_{a}^{b} f_{0}(t) d t=\frac{1}{4} k(b-a)^{2} .
$$

If in (10) we choose $f_{0}$ as above and $x=\frac{a+b}{2}$ then we get

$$
C\left[\frac{1}{4}(b-a)^{2} k+\frac{1}{4}(b-a)^{2} k\right] \leq \frac{1}{4} k(b-a)^{2},
$$

which implies $C \leq \frac{1}{2}$, and the sharpness of the constant is proved.

Also solved by Jim Bradley (Devon, UK), John N. Lillington (Wareham, UK)

104. Let $f:[a, b] \rightarrow \mathbb{R}$ be a convex function defined on the closed interval $[a, b]$. Prove that for any $x \in[a, b]$, the following holds

$$
\int_{a}^{b} f(t) d t-(b-a) f(x) \leq \frac{1}{2}\left[(b-x)^{2} f_{-}^{\prime}(b)-(x-a)^{2} f_{+}^{\prime}(a)\right] .
$$

The constant $\frac{1}{2}$ on the right side of (2) is sharp in the sense that it cannot be replaced by a smaller constant.

(Sever S.Dragomir, Victoria University, Australia)
Solution by the proposer. If either $f_{+}^{\prime}(a)=-\infty$ or $f_{-}^{\prime}(b)=+\infty$ then inequality (11) evidently holds true. Assume that $f_{+}^{\prime}(a)$ and $f_{-}^{\prime}(b)$ are finite. Since $f$ is convex on $[a, b]$, we have

$$
f^{\prime}(t) \geq f_{+}^{\prime}(a) \text { for a.e. } t \in[a, x]
$$

and

$$
f^{\prime}(t) \leq f_{-}^{\prime}(b) \text { for a.e. } t \in[x, b] .
$$

If we multiply (12) by $t-a \geq 0, t \in[a, x]$, and integrate on $[a, x]$ then we deduce

$$
\int_{a}^{x}(t-a) f^{\prime}(t) d t \geq \frac{1}{2}(x-a)^{2} f_{+}^{\prime}(a)
$$

and if we multiply (13) by $b-t \geq 0, t \in[x, b]$, and integrate on $[x, b]$ then we also have

$$
\int_{x}^{b}(b-t) f^{\prime}(t) d t \leq \frac{1}{2}(b-x)^{2} f_{-}^{\prime}(b) .
$$

Finally, if we subtract (14) from (15) and use the representation

$$
\int_{a}^{x}(t-a) f^{\prime}(t) d t+\int_{x}^{b}(t-b) f^{\prime}(t) d t=f(x)-\int_{a}^{b} f(t) d t
$$

we deduce the desired inequality (11).

Now, assume that (11) holds with a constant $D>0$ instead of $\frac{1}{2}$, that is,

$$
\int_{a}^{b} f(t) d t-(b-a) f(x) \leq D\left[(b-x)^{2} f_{-}^{\prime}(b)-(x-a)^{2} f_{+}^{\prime}(a)\right] .
$$

If we consider the convex function $f_{0}:[a, b] \rightarrow \mathbb{R}$ defined by

$$
f_{0}(t)=k\left|t-\frac{a+b}{2}\right|
$$

then we have $f_{-}^{\prime}(b)=k, f_{+}^{\prime}(a)=-k$ and by (16) we deduce for $x=\frac{a+b}{2}$ that

$$
\frac{1}{4} k(b-a)^{2} \leq D\left[\frac{1}{4} k(b-a)^{2}+\frac{1}{4} k(b-a)^{2}\right],
$$

giving $D \geq \frac{1}{2}$, and the sharpness of the constant is proved.

Also solved by Jim Bradley (Devon, UK), John N. Lillington (Wareham, UK)

Open problem $105^{*}$. Let $A_{n}=\left(a_{i j}\right)$ be a real matrix of order $n$, with

$$
a_{i j}=\sum_{k=1}^{j}(-1)^{k} \cos ^{2 i}\left(\frac{k}{j+1} \frac{\pi}{2}\right)
$$

and let $B_{n}=\left(b_{i j}\right)$ be the inverse of $A_{n}$. Prove that $b_{i j}=0$, for $j>i+1$.

(Carlos M. da Fonseca, University of Coimbra, Portugal)

Solution by Mircea Merca, University of Craiova, Romania

Let $A_{n}^{\prime}=\left(a_{i, j}\right)$ be an $n \times n$ matrix of the form

$$
A_{n}^{\prime}=\left(\begin{array}{ccccc}
x & x & \cdots & x & x \\
a_{2,1}^{\prime} & x & \cdots & x & x \\
\vdots & \ddots & \ddots & \vdots & \vdots \\
\vdots & \ddots & \ddots & x & x \\
a_{n, 1}^{\prime} & \cdots & \cdots & a_{n, n-1}^{\prime} & x
\end{array}\right),
$$


with $x \neq 0$. It is clear that

$$
\begin{aligned}
\operatorname{det} A_{n}^{\prime} & =\left|\begin{array}{ccccc}
x & x & \cdots & x & x \\
a_{2,1}^{\prime}-x & 0 & \cdots & 0 & 0 \\
\vdots & \ddots & \ddots & \vdots & \vdots \\
\vdots & \ddots & \ddots & 0 & 0 \\
a_{n, 1}^{\prime}-x & \cdots & \cdots & a_{n, n-1}^{\prime}-x & 0
\end{array}\right| \\
& =(-1)^{n+1} x \prod_{i=1}^{n-1}\left(a_{i+1, i}^{\prime}-x\right) .
\end{aligned}
$$

If $a_{i+1, i}^{\prime}=x$ for at least one $i<n$ then we have $\operatorname{det} A_{n}^{\prime}=0$. We assume that $a_{i+1, i}^{\prime} \neq x$ for all $i<n$. Let $B_{n}^{\prime}$ be the inverse of $A_{n}^{\prime}$,

$$
B_{n}^{\prime}=\frac{1}{\operatorname{det} A_{n}^{\prime}}\left(C_{i, j}\right)
$$

where $\left(C_{i, j}\right)$ is the adjoint of $A_{n}^{\prime}$. For $i<j-1$ it is clear that $C_{i, j}$ is the determinant of an $(n-1) \times(n-1)$ matrix of the form (17), with $a_{i+1, i}^{\prime}=x$ for at least one $i<n-1$. Thus, we deduce that $C_{i, j}=0$ for $i<j-1$.
By Merca [1, Theorem 2], we easily deduce that $A_{n}$ is a matrix of the form (17) with $x=-\frac{1}{2}$ and $a_{i+1, i} \neq x$ for $i<n$, that is,

$$
a_{i+1, i}=\frac{i+1}{2^{2 i+1}}-\frac{1}{2} .
$$

References

[1] M. Merca, A Note on Cosine Power Sums, J. Integer Seq., 15(5) (2012), Article 12.5.3.

Also solved by Christian Krattenthaler (Faculty of Mathematics, University of Wien, Austria)

We wait to receive your solutions to the proposed problems and ideas on the open problems. Send your solutions both by ordinary mail to Themistocles M. Rassias, Department of Mathematics, National Technical University of Athens, Zografou Campus, GR 15780, Athens, Greece, and by email to trassias@math.ntua.gr.

We also solicit your new problems with their solutions for the next "Solved and Unsolved Problems" column, which will be devoted to Analytic Inequalities.

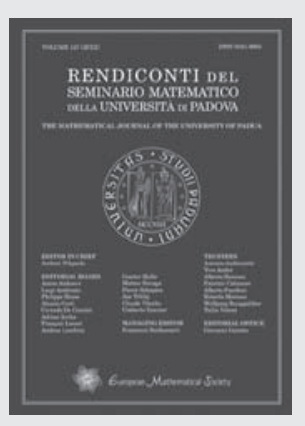

ISSN print 0041-8994

ISSN online $2240-2926$

2013. 2 volumes (129 and 130)

of approx. 250 pages each

$17.0 \times 24.0 \mathrm{~cm}$

Price of subscription:

198 € online only

238 € print+online

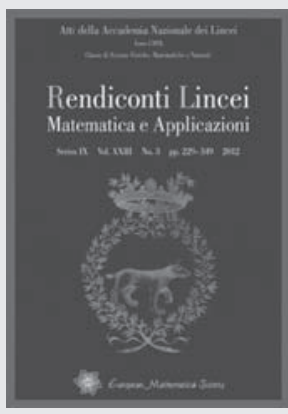

ISSN print $\quad 1120-6330$

ISSN online $1720-0768$

2013. Series 9 Vol. 23. 4 issues

Approx. 400 pages

$17.0 \times 24.0 \mathrm{~cm}$

Price of subscription:

$200 €$ online only

240 € print+online

Reduced subscription rate for

Italian end customers
A journal of the Seminario Matematico della Università di Padova

\section{Editor-in-Chief:}

Andrea D'Agnolo (Università di Padova, Italy)

Managing Editor:

Francesco Baldassarri (Università di Padova, Italy)

\section{Aims and Scope}

The aim of Rendiconti is to publish original high-quality research articles in mathematics. By tradition the journal focuses on algebra, analysis, algebraic and differential geometry, mathematical physics and number theory. The journal is ready to accept long papers of foundational or technical nature, as well as papers containing applications or surveys, provided these are especially well structured and fill a gap in the literature.

Atti della Reale Accademia dei Lincei

\section{Director Advisory Committee:}

A. Ambrosetti (Trieste)

\section{Aims and Scope}

The Accademia dei Lincei (Lynx), founded in 1603, is the oldest academy dedicated to the study of humanities as well as physics, mathematics and the natural sciences in the world. Through the centuries, some of the most important scientists of their time have been among their members, including Galileo Galilei, Enrico Fermi and Vito Volterra.

Rendiconti Lincei is dedicated to the publication of high-quality peer-reviewed surveys, research papers and preliminary announcements of important results from all fields of mathematics and its applications.
European Mathematical Society Publishing House

Seminar for Applied Mathematics, ETH-Zentrum SEW A27
Scheuchzerstrasse 70

CH-8092 Zürich, Switzerland subscriptions@ems-ph.org

www.ems-ph.org 


\section{American Mathematical Society}
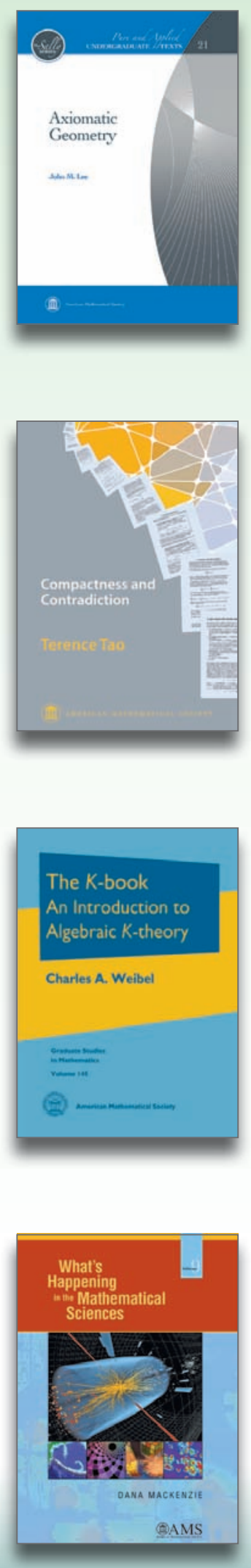

\section{AXIOMATIC GEOMETRY}

John M. Lee, University of Washington

The story of geometry is the story of mathematics itself: Euclidean geometry was the first branch of mathematics to be systematically studied and placed on a firm logical foundation, and it is the prototype for the axiomatic method that lies at the foundation of modern mathematics. It has been taught to students for more than two millennia as a model of logical thought. This book tells the story of how the axiomatic method has progressed from Euclid's time to ours, as a way of understanding what mathematics is, how we read and evaluate mathematical arguments, and why mathematics has achieved the level of certainty it has.

Pure and Applied Undergraduate Texts, Vol. 21

May 2013 473pp 9780821884782 Hardback $€ 66.00$

\section{COMPACTNESS AND CONTRADICTION}

Terence Tao, University of California

There are many bits and pieces of folklore in mathematics that are passed down from advisor to student, or from collaborator to collaborator, but which are too fuzzy and nonrigorous to be discussed in the formal literature. Traditionally, it was a matter of luck and location as to who learned such "folklore mathematics". But today, such bits and pieces can be communicated effectively and efficiently via the semiformal medium of research blogging. This book grew from such a blog.

Apr 2013 256pp 9780821894927 Paperback $€ 39.00$

\section{THE $K$-BOOK}

\section{An Introduction to Algebraic K-theory}

Charles A. Weibel, Rutgers University

A comprehensive introduction to the subject of algebraic $K$-theory. This book blends classical algebraic techniques for $K_{0}$ and $\mathrm{K}_{1}$ with newer topological techniques for higher $K$-theory such as homotopy theory, spectra, and cohomological descent. The book takes the reader from the basics of the subject to the state of the art, including the calculation of the higher $K$-theory of number fields and the relation to the Riemann zeta function.

Graduate Studies in Mathematics, Vol. 145

May 2013 642pp 9780821891322 Hardback $€ 79.00$

\section{WHAT'S HAPPENING IN THE MATHEMATICAL SCIENCES Volume 9}

\section{Dana Mackenzie}

What's Happening in the Mathematical Sciences looks at some highlights of the most recent developments in mathematics. These include the mathematics behind stories that made headlines, as well as fascinating mathematical stories that never made it into the newspapers.

What's Happening in the Mathematical Sciences, Vol. 9

May 2013 136pp 9780821887394 Paperback $€ 22.00$

\section{To order AMS titles visit www.eurospanbookstore.com/ams}

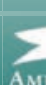

$125^{\text {th }}$ Anniversary $<$ AmERican MATHEMATICAL SOCIETY 


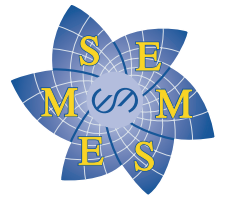

\section{New books published by the}

\section{uropean Mathematical Society}

Individual members of the EMS, member societies or societies with a reciprocity agreement (such as the American, Australian and Canadian Mathematical Societies) are entitled to a discount of $20 \%$ on any book purchases, if ordered directly at the EMS Publishing House.

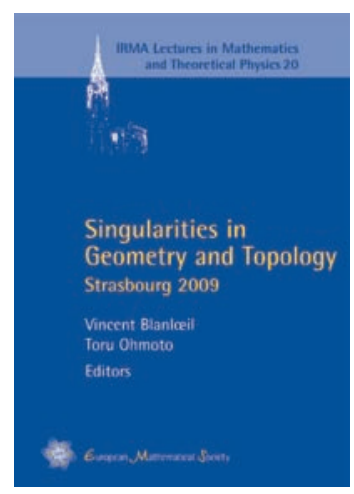

Vincent Blanlœil (Université de Strasbourg, France) and Toru Ohmoto (Hokkaido University, Sapporo, Japan) Singularities in Geometry and Topology. Strasbourg 2009 (IRMA Lectures in Mathematics and Theoretical Physics, Vol. 20)

978-3-03719-118-7. 2012. 370 pages. Softcover. 17 x 24 cm. 48.00 Euro

This volume arises from the 5th Franco-Japanese Symposium on Singularities, held in Strasbourg in August 2009. The conference brought together an international group of researchers working on singularities in algebraic geometry, analytic geometry and topology, mainly from France and Japan. Besides, it also organized a special session, JSPS Forum on Singularities and Applications, which was aimed to introduce some recent applications of singularity theory to physics and statistics.

The book comprises research papers and short lecture notes on advanced topics on singularities. Some surveys on applications that were presented in the Forum are also added. Topics covered include splice surface singularities, $b$-functions, equisingularity, degenerating families of Riemann surfaces, hyperplane arrangements, mixed singularities, jet schemes, noncommutative blow-ups, characteristic classes of singular spaces, and applications to geometric optics, cosmology and learning theory.

Graduate students who wish to learn about various approaches to singularities, as well as experts in the field and researchers in other areas of mathematics and science will find the contributions to this volume a rich source for further study and research.

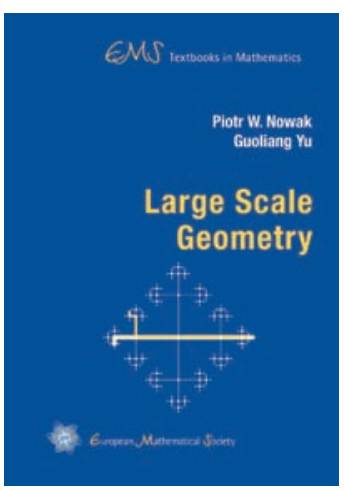

Piotr W. Nowak (IMPAN, Warsaw, Poland) and Guoliang Yu (Texas A\&M University, College Station, USA)

Large Scale Geometry (EMS Textbooks in Mathematics)

ISBN 978-3-03719-112-5. 2012. 203 pages. Hardcover. 16.5 x 23.5 cm. 38.00 Euro

Large scale geometry is the study of geometric objects viewed from a great distance. The idea of large scale geometry can be traced back to Mostow's work on rigidity and the work of Švarc, Milnor and Wolf on growth of groups. In the last decades, large scale geometry has found important applications in group theory, topology, geometry, higher index theory, computer science, and large data analysis. This book provides a friendly approach to the basic theory of this exciting and fast growing subject and offers a glimpse of its applications to topology, geometry, and higher index theory. The authors have made a conscientious effort to make the book accessible to advanced undergraduate students, graduate students, and non-experts.

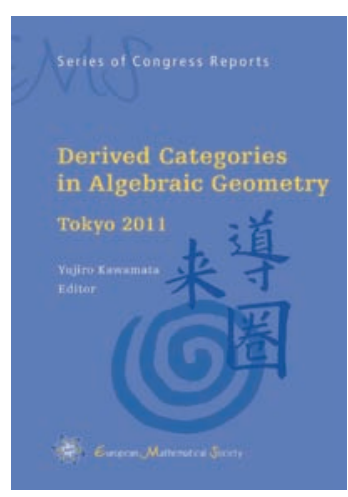

Yujiro Kawamata (University of Tokyo, Japan)

Derived Categories in Algebraic Geometry. Tokyo 2011 (EMS Series of Congress Reports)

ISBN 978-3-03719-115-6. 2013. 354 pages. Hardcover. 17 x 24 cm. 78.00 Euro

The study of derived categories is a subject that attracts increasingly many young mathematicians from various fields of mathematics, including abstract algebra, algebraic geometry, representation theory and mathematical physics.

The concept of the derived category of sheaves was invented by Grothendieck and Verdier in the 1960s as a tool to express important results in algebraic geometry such as the duality theorem. In the 1970s, Beilinson, Gelfand and Gelfand discovered that a derived category of an algebraic variety may be equivalent to that of a finite dimensional non-commutative algebra, and Mukai found that there are nonisomorphic algebraic varieties that have equivalent derived categories. In this way the derived category provides a new concept that has many incarnations. In the 1990s, Bondal and Orlov uncovered an unexpected parallelism between derived categories and birational geometry. Kontsevich's homological mirror symmetry provided further motivation for the study of derived categories.

This book is the proceedings of a conference held at the University of Tokyo in January 2011 on the current status of the research on derived categories related to algebraic geometry. Most articles are survey papers on this rapidly developing field. The book is suitable for young mathematicians who want to enter this exciting field. Some basic knowledge of algebraic geometry is assumed.

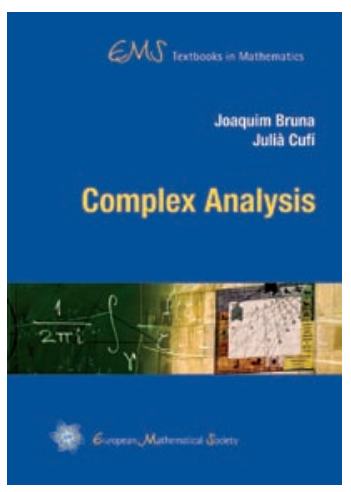

Joaquim Bruna and Julià Cufí (both Universitat Autònoma de Barcelona, Spain)

Complex Analysis (EMS Textbooks in Mathematics)

ISBN 978-3-03719-111-8. 2013. Approx. 592 pages. Hardcover. 16.5 x 23.5 cm. 58.00 Euro

The theory of functions of a complex variable is a central theme in mathematical analysis that has links to several branches of mathematics. Understanding the basics of the theory is necessary for anyone who wants to have a general mathematical training or for anyone who wants to use mathematics in applied sciences or technology.

The book presents the basic theory of analytic functions of a complex variable and their points of contact with other parts of mathematical analysis. This results in some new approaches to a number of topics when compared to the current literature on the subject.

The text can be used as a manual for complex variable courses of various levels and as a reference book. The only prerequisites for reading it is a working knowledge of the topology of the plane and the differential calculus for functions of several real variables. A detailed treatment of harmonic functions also makes the book useful as an introduction to potential theory. 